\title{
Branched coverings and cubic Newton maps
}

by

\author{
Lei Tan (Warwick)
}

\begin{abstract}
We construct branched coverings such as matings and captures to describe the dynamics of every critically finite cubic Newton map. This gives a combinatorial model of the set of cubic Newton maps as the gluing of a subset of cubic polynomials with a part of the filled Julia set of a specific polynomial (Figure 1).
\end{abstract}

Introduction. The Newton's method of a polynomial $P$ is defined to be the following rational map:

$$
N_{P}: \overline{\mathbb{C}} \rightarrow \overline{\mathbb{C}}, \quad z \mapsto z-\frac{P(z)}{P^{\prime}(z)}
$$

A cubic Newton map $N$ is a rational map conformally conjugate to $N_{P}$, with $P$ a cubic polynomial with simple roots. The map $N$ has four critical points. Three of them are also fixed points. They are the zeros of $P$. The fourth one, denoted by $x_{0}$, can have various dynamics under the iterations of $N$. We say that $N$ is postcritically finite if $x_{0}$ has a finite orbit.

In this paper we intend to give three ways (one due to J. Head) to construct a branched covering which is equivalent (in the sense of Thurston) to a postcritically finite cubic Newton map. The first one (at the end of Section 3) is the simplest and produces many interesting examples. The second one (matings) together with the third one (captures, due to J. Head) will cover all postcritically finite cubic Newton maps. Here is a more precise statement:

For a generic cubic Newton map $N_{P}$, there is a unique, dynamically determined numbering $p_{1}, p_{2}$ and $p_{3}$ of the three roots of $P$ in the following way: in the Böttcher coordinates of immediate basins $A_{1}, A_{2}$ and $A_{3}$, the three 0-rays land at $\infty$ in counterclockwise order, and the $1 / 2$-rays of $A_{1}$ and $A_{2}$ land at the same point (see Section 2.1 for details). Define $\mathbf{f}$ to be the specific polynomial $z \mapsto z^{3}+(3 / 2) z$. We will prove

1991 Mathematics Subject Classification: Primary 58F23. 
MaIn Theorem. There is a subset $A$ of cubic polynomials and a subset $Y$ of the filled Julia set of $\mathbf{f}$ such that: there is a surjective mapping $\mathcal{M}$ from $A \cup Y$ onto the set of postcritically finite cubic Newton maps (up to conformal conjugacy) such that: for any $g \in A($ resp. $y \in Y)$, the Newton map $\mathcal{M}(g)$ (resp. $\mathcal{M}(y)$ ) is equivalent to the mating $\mathbf{f} \Perp g$ (resp. the capture $\left.F_{y}\right)$. Moreover:

1. Any map $N_{P}$ with $x_{0}$ either periodic (but not fixed) or a preimage of $p_{1}$ is equivalent to a unique mating (i.e. is in $\mathcal{M}(A)$ with a unique $g \in A$ ).

2. Any map $N_{P}$ with $x_{0}$ a preimage of $p_{2}$ or $p_{3}$ is equivalent to a capture (i.e. is in $\mathcal{M}(Y)$ ).

3. Any other postcritically finite $N_{P}$ is equivalent to a capture and a mating (i.e. is in $\mathcal{M}(A) \cap \mathcal{M}(Y)$ ), with two choices of $g \in A$ in case $x_{0}$ is a preimage of $\infty$, or a unique $g \in A$ otherwise.

Therefore, the space of cubic Newton maps can be considered (combinatorially) as the gluing of a subset of cubic polynomials with a part of the filled Julia set of $\mathbf{f}$. This is one of the rare families of maps that can be completely described by matings and captures. Even the family of quadratic rational maps with one superattracting cycle of period 3 is more complicated.

Head's angle of a cubic Newton map is a very important information about the location of the free critical point $x_{0}$. Conjecturally the Head's angle characterises the islands appearing in the computer generated picture of the parameter space. We will apply the Main Theorem to characterise Newton maps with a given Head angle by means of matings and captures (Proposition 6.3).

This result gives rise to another classification of the cubic Newton family by abstract graphs. More precisely, there is a space of abstract graphs, each corresponding to a unique cubic Newton map $N$ (up to conformal conjugacy), in the sense that $N$ has a forward invariant topological finite graph, which contains the orbit of the critical points, and which is homeomorphic to the given abstract graph. We also know exactly when two graphs give the same rational map.

Section 1 gives a parametrization of the set of cubic Newton maps (the $\lambda$-plane), together with a description of hyperbolic components, the location of the three main components $B_{0}, G_{0}, R_{0}$ and their 0 - and 1/2-rays, and a fundamental domain $\Omega$ for conformal classes of cubic Newton maps. It ends with a corollary of the main theorem in terms of parameters.

Section 2 states first some general results on the dynamics of a cubic Newton map, and describes the dynamical numbering of the three roots (mainly due to J. Head). As a simple consequence, we show that the Julia set is always connected. We also state the conditions for the boundary of an attracting basin to be a Jordan curve (with a proof by M. Shishikura). 
It is followed by a combinatorial study of an open subset of $\mathbb{R} / \mathbb{Z}$, which is considered to be a combinatorial model via Head's angles of the connected components of $\mathbb{C}-\overline{B_{0} \cup G_{0} \cup R_{0}}$ (the islands). We then state the definition and main topological properties of Head's angles. It will be shown at the end of Section 3 that any branched covering satisfying these properties is in fact equivalent to a cubic Newton map.

Section 3 contains the key step towards our branched covering constructions. There we simplify a Thurston obstruction $\Gamma$ to a one-curve Levy cycle, if $\Gamma$ occurs for a cubic branched covering having three fixed critical points.

The results about matings are contained in Section 4. It determines the set $A$ by means of dynamical properties and proves the mating part of the Main Theorem. By using Head's results about captures, we define in Section 5 the set $Y$ as a subset of the filled Julia set of $\mathbf{f}$ and prove the capture part of the Main Theorem.

Finally, in Section 6 we interpret the sets $A$ and $Y$ in parameter spaces and give more precise information about the gluing (Theorem 6.1). We will see that $A$ is precisely the postcritically finite locus in a quarter part of the following space: the connectedness locus of the family $g_{a}: z \mapsto$ $z^{3}-3 a^{2} z+2 a^{3}+a$ minus the "head" and the "foot" part, and $Y$ is the preperiodic locus in a quarter part of the following space: the filled Julia set of $\mathbf{f}$ minus the "head" and the "foot" part. Moreover, $\mathcal{M}(A) \cup \mathcal{M}(Y)$ can be realized as $A \sqcup Y$ quotiented by ray-equivalence relations. Figure 1 is a schematic picture of these sets and their gluings.

This result on postcritically finite maps suggests the following conjecture: The fundamental part of the cubic Newton family is homeomorphic to the quotient of a well determined subset of the $a$-family union a specific subset of the filled Julia set of $\mathbf{f}$, by the equivalence relation generated by external rays.

The paper ends with a discussion of graph classifications and Head's angle determination via matings and captures.

The author would like to thank all the people who have had discussions with her and encouraged her, especially B. Branner, A. Douady, F. von Haeseler, J. H. Hubbard, J. W. Milnor, C. L. Petersen, M. Rees, P. Sentenac, B. Sevennec and M. Shishikura. The author would also like to thank Bremen University where the first part of the present work was done. Many thanks are due to Madame Blandine Mauduit for the drawing of all the schematic pictures in the preprint version of the paper (prépublication ENS Lyon, no. 26, 1990). Finally, it is a pleasure to thank D. Faught and J. Smillie for having read carefully the manuscript.

Figure 3 (resp. Figure 4, Figure 5) shows the Julia set of $N_{\lambda}$ for $\lambda=a$ (resp. $\lambda=b, \lambda=c)$. We have $N_{a}^{2}\left(x_{0}\right)=p_{3}=1\left(\operatorname{resp} . N_{b}^{3}\left(x_{0}\right)=p_{1}=\right.$ 
$\left.-1 / 2-b, N_{c}^{2}\left(x_{0}\right)=x_{0}\right)$. Note that $c$ is in the center of a small Mandelbrot set. Further pictures of related Julia sets and parameter spaces can be found in [CGS], [DH3], [He], [HP] and [Sa].

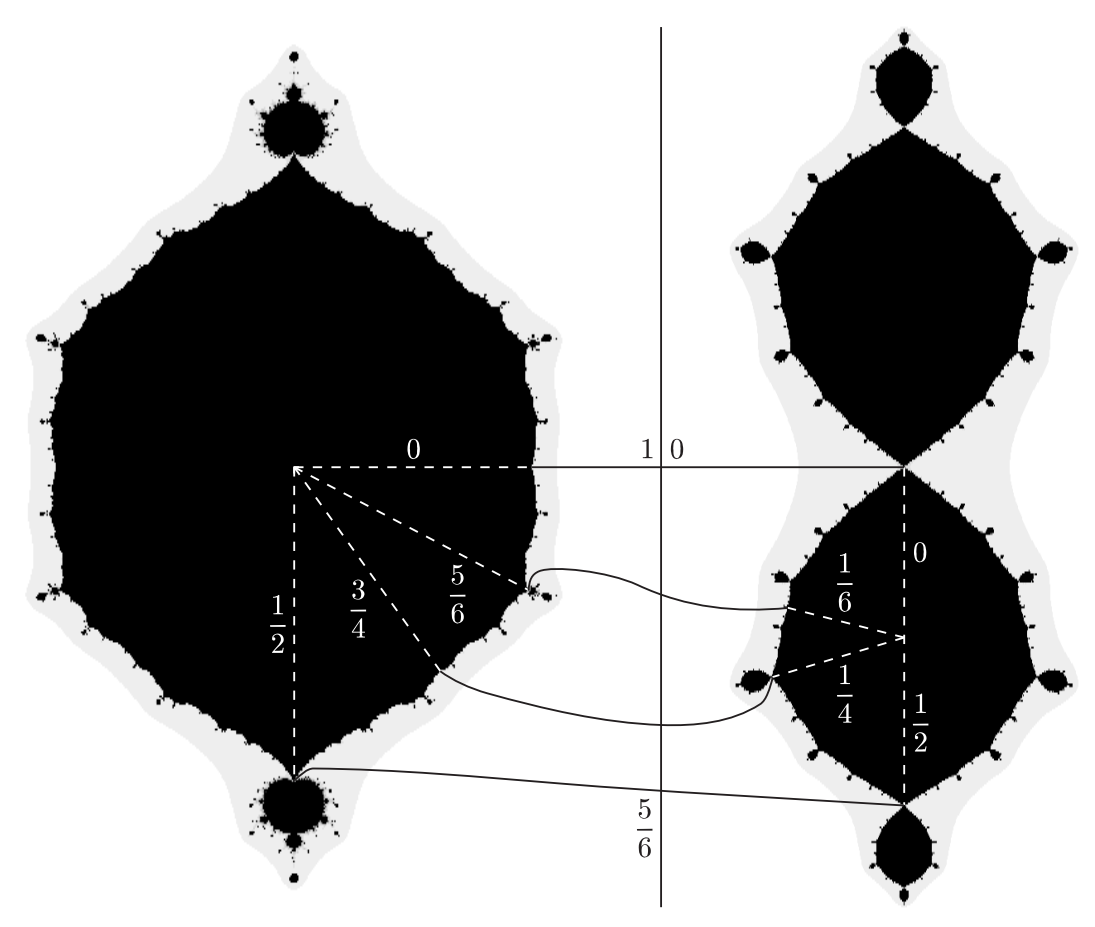

Fig. 1. How to mate in the parameter spaces
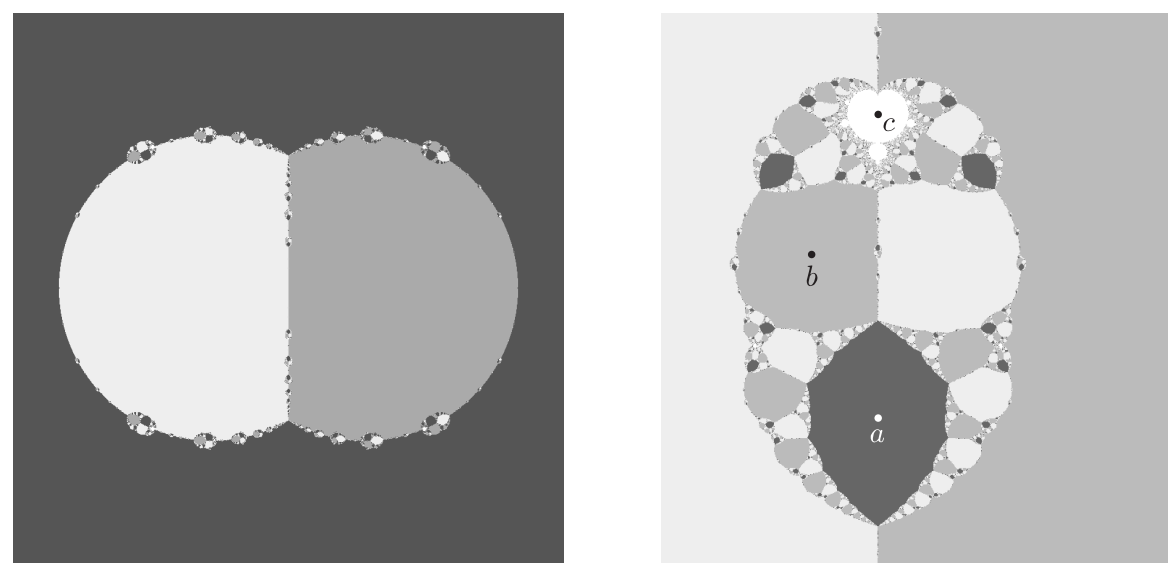

Fig. 2. The parameter $\lambda$-plane and a magnification around an island 


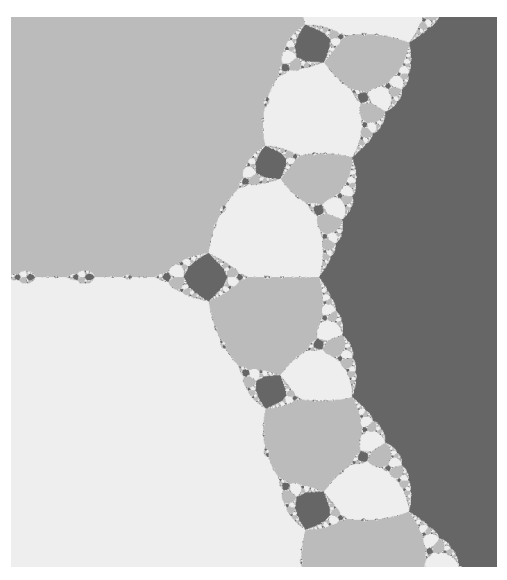

Fig. 3

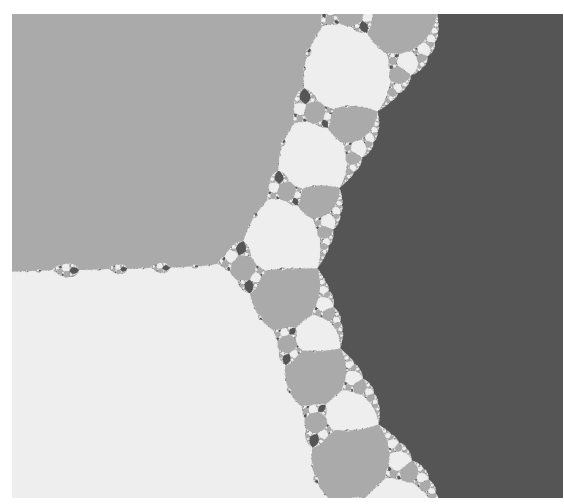

Fig. 4

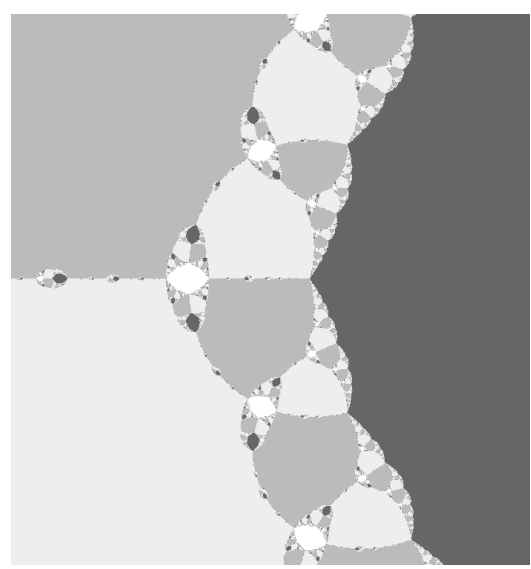

Fig. 5 
1. Description of the parameter space. For definitions of Julia set, filled Julia set, critical point, repulsive (attracting, superattracting, indifferent) periodic point, (immediate) attracting basin, external angles of a monic polynomial, etc., we refer the reader for example to [B] and [DH1].

Let $P: \mathbb{C} \rightarrow \mathbb{C}$ be a monic complex polynomial of degree $d$. Define

$$
N_{P}: \overline{\mathbb{C}} \rightarrow \overline{\mathbb{C}}, \quad z \mapsto z-\frac{P(z)}{P^{\prime}(z)},
$$

where $P^{\prime}(z)$ denotes the derivative of $P . \quad N_{P}$ is called Newton's method of $P$. It is well known and easy to check that the simple roots of $P$ are superattracting fixed points of $N_{P}$.

Note first that given two polynomials $P, Q$, a necessary and sufficient condition for their Newton's methods to be conjugate by a Möbius transformation (i.e. $N_{Q}=\varphi^{-1} \circ N_{P} \circ \varphi$ ) is $Q(z)=K \cdot P(a z+b)$, where $K, a, b$ are some complex numbers. Moreover, the conjugacy mapping $\varphi$ is exactly $z \mapsto a z+b$. Hence for any cubic polynomial $Q$ the Newton's method $N_{Q}$ is conjugate to a rational map of the following form:

$$
N_{\lambda}: z \mapsto \frac{2 z^{3}+\left(1 / 4-\lambda^{2}\right)}{3 z^{2}-\left(3 / 4+\lambda^{2}\right)}=\frac{2 z^{3}+\sigma_{3}}{3 z^{2}+\sigma_{2}},
$$

which is the Newton's method of the polynomial

$$
P_{\lambda}: z \mapsto(z+1 / 2-\lambda)(z+1 / 2+\lambda)(z-1)=\left(z-r_{1}\right)\left(z-r_{2}\right)\left(z-r_{3}\right)
$$

with $\sigma_{1}=r_{1}+r_{2}+r_{3}=0, \sigma_{2}=r_{1} r_{2}+r_{2} r_{3}+r_{1} r_{3}$ and $\sigma_{3}=r_{1} r_{2} r_{3}$. Moreover, $N_{\lambda}$ and $N_{\lambda^{\prime}}$ are conjugate iff $G(\lambda)=G\left(\lambda^{\prime}\right)$, where

$$
G: \mathbb{C} \rightarrow \mathbb{C}, \quad \lambda \mapsto \sigma_{2}^{3} / \sigma_{3}^{2} .
$$

Note that in particular $N_{\lambda}$ and $N_{-\lambda}$ are conjugate, and $N_{\lambda}$ and $N_{\bar{\lambda}}$ are conjugate by $z \mapsto \bar{z}$.

The critical points of $G$ are $\infty, \pm 3 / 2, \pm 1 / 2,0, \pm i \sqrt{3} / 2$ (where $\pm i \sqrt{3} / 2$ are double critical points). We have

$$
G( \pm 3 / 2)=G(0)=-27 / 4, \quad G( \pm 1 / 2)=G(\infty)=\infty, \quad G( \pm i \sqrt{3} / 2)=0 .
$$

Moreover,

$$
\begin{aligned}
G^{-1}([-\infty,-27 / 4])= & \mathbb{R}, \\
G^{-1}([-27 / 4,0])= & \{i y|| y \mid \leq \sqrt{3} / 2\} \cup\left\{-1 / 2+e^{i \theta} \mid \pi / 3 \leq \theta \leq 5 \pi / 3\right\} \\
& \cup\left\{1 / 2+e^{i \theta} \mid-2 \pi / 3 \leq \theta \leq 2 \pi / 3\right\}, \\
G^{-1}([0,+\infty])= & \{i y|| y \mid>\sqrt{3} / 2\} \cup\left\{-1 / 2+e^{i \theta}|| \theta \mid \leq \pi / 3\right\} \\
& \cup\left\{1 / 2+e^{i \theta}|| \theta \mid \geq 2 \pi / 3\right\} .
\end{aligned}
$$

Special cases:

1. $\lambda= \pm 1 / 2$; then $N_{\lambda}$ is conjugate to $N_{P}$ for $P: z \mapsto z^{3}-z$. 
2. $\lambda=0, \pm 3 / 2$; then $P_{\lambda}$ has a double root and $N_{\lambda}$ is a rational map of lower degree.

3. $\lambda= \pm i \sqrt{3} / 2$; then $N_{\lambda}$ is conjugate to $N_{P}$ for $P: z \mapsto z^{3}-1$.

Therefore the map

$$
\lambda \mapsto \frac{1}{1+\frac{4}{27} G(\lambda)}
$$

gives rise to an isomorphism between the space of cubic Newton maps up to conformal conjugacy and $\mathbb{C}$.

The mapping $N_{\lambda}$ has four critical points $-1 / 2+\lambda,-1 / 2-\lambda, 1$ and $x_{0}=0$. Among them $-1 / 2+\lambda,-1 / 2-\lambda, 1$ are fixed by $N_{\lambda}$. Only $x_{0}$ can have a free dynamics. We can then understand the dynamics of $N_{\lambda}$ by following the dynamics of $x_{0}$.

Denote by $A_{\lambda}^{+}$(resp. $\left.A_{\lambda}^{-}, A_{\lambda}^{1}\right)$ the immediate attracting basin for $N_{\lambda}$ of $-1 / 2+\lambda$ (resp. of $-1 / 2-\lambda, 1)$. Figure 2 is a computer generated picture of the parameter space. The point $\lambda$ is left light grey (resp. grey, dark grey) if $N_{\lambda}^{n}\left(x_{0}\right)$ converges to $-1 / 2-\lambda$ (resp. $-1 / 2+\lambda, 1$ ), and is colored white if not.

Define $\lambda$ to be a hyperbolic point if $N_{\lambda}^{n}\left(x_{0}\right)$ converges to an attracting periodic orbit. The set of hyperbolic points forms an open subset of $\mathbb{C}$. Its connected components are called hyperbolic components. It is conjectured that hyperbolic points form a dense set. The following analysis is very similar to the analysis of the family $S_{1}$ in [F], [M1].

There are several types of hyperbolic components:

Type A (adjacent critical points):

$B_{0}$ : with the critical point $x_{0}$ in the immediate attracting basin $A_{\lambda}^{+}$of $-1 / 2+\lambda$. There is only one such hyperbolic component.

$G_{0}$ : with the critical point $x_{0}$ in the immediate attracting basin $A_{\lambda}^{-}$of $-1 / 2-\lambda$. There is only one such hyperbolic component.

$R_{0}$ : with the critical point $x_{0}$ in the immediate attracting basin $A_{\lambda}^{1}$ of 1. There is only one such hyperbolic component. As a convention, we assume $\infty \in R_{0}$.

Type C (capture):

$C^{+}$: with $N_{\lambda}^{n}\left(x_{0}\right)$ converging to $-1 / 2+\lambda$ but $x_{0} \notin A_{\lambda}^{+}$.

$C^{-}$: with $N_{\lambda}^{n}\left(x_{0}\right)$ converging to $-1 / 2-\lambda$ but $x_{0} \notin A_{\lambda}^{-}$.

$C^{1}$ : with $N_{\lambda}^{n}\left(x_{0}\right)$ converging to 1 but $x_{0} \notin A_{\lambda}^{1}$.

Type D (disjoint attracting orbits): with $N_{\lambda}^{n}\left(x_{0}\right)$ converging to an attracting orbit disjoint from $\{-1 / 2+\lambda,-1 / 2-\lambda, 1\}$.

In all cases, the same proof as Milnor's will show that each hyperbolic component (except $R_{0}$ ) is an open topological 2-cell, and contains a unique 
$\lambda$ such that $N_{\lambda}$ is postcritically finite. As a consequence, the set of nonhyperbolic points is connected. It is conjectured to be also locally connected, and without interior.

The following lemmas, whose proof uses some results in Section 2, explain the half apple shape in $\lambda$-plane. Denote by $[-i \sqrt{3} / 2, i \sqrt{3} / 2]$ the set $\{i y \mid$ $|y| \leq \sqrt{3} / 2\}$.

Lemma 1.1. The imaginary axis $\{\lambda \mid \operatorname{Re}(\lambda)=0\}$ can only intersect hyperbolic components of type $R_{0}, D$ and $C^{1}$. Moreover, $\{\lambda \mid \operatorname{Re}(\lambda)=0\}-R_{0}$ $=[-i \sqrt{3} / 2, i \sqrt{3} / 2]$.

Pro of. Since $N_{\lambda}$ is a function of $\lambda^{2}$, it is real when $\operatorname{Re}(\lambda)=0$. When $\operatorname{Re}(\lambda)=0$, the two components $A_{\lambda}^{+}$and $A_{\lambda}^{-}$are symmetric to each other with respect to the real axis so do not intersect the real axis, and $A_{\lambda}^{1}$ is symmetric to itself with respect to the real axis. Since $x_{0}=0$ is also real, $N_{\lambda}^{k}\left(x_{0}\right)$ remains real for every integer $k>0$, and is not attracted by either $-1 / 2+\lambda$ or $-1 / 2-\lambda$. Hence $\lambda \notin B_{0} \cup G_{0}$ and $\lambda$ cannot be in a hyperbolic component of type $C^{+}, C^{-}$.

The other two preimages of $\infty$ are $\xi_{1,2}= \pm \sqrt{1 / 4+\lambda^{2} / 3}$. Set $\lambda=r i$, with $r \in \mathbb{R}$. According to Lemmas 2.3 and 2.5 below, the component $A_{\lambda}^{1}$ is connected, at least one of $\xi_{1}, \xi_{2}$ is contained in $\partial A_{\lambda}^{1}$, and $x_{0} \in A_{\lambda}^{1}$ if and only if both $\xi_{1}, \xi_{2} \in \partial A_{\lambda}^{1}$. In case $r^{2}<3 / 4, \xi_{1,2}$ are real. Since $A_{\lambda}^{1}$ is symmetric with respect to the real axis, the set $A_{\lambda}^{1} \cap \mathbb{R}$ is connected. So only one of $\xi_{1,2}$ can appear in $\partial A_{\lambda}^{1}$. We then have $x_{0} \notin A_{\lambda}^{1}$. In case $r^{2}>3 / 4$, $\xi_{1,2}$ are complex conjugate. They must both appear in $\partial A_{\lambda}^{1}$ because of the symmetry. We then have $x_{0} \in A_{\lambda}^{1}$. In case $r^{2}=3 / 4$, we have $x_{0}=\xi_{1}=\xi_{2} \in$ $\partial A_{\lambda}^{1}$, hence $x_{0} \notin A_{\lambda}^{1}$. This proves $\{\lambda \mid \operatorname{Re}(\lambda)=0\}-R_{0}=[-i \sqrt{3} / 2, i \sqrt{3} / 2]$.

LEMMA 1.2. Each of the three components of $\mathbb{C}-G^{-1}([-27 / 4,0])$ contains one of $B_{0}, G_{0}$ and $R_{0}$, with $1 / 2 \in B_{0},-1 / 2 \in G_{0}$ and $R_{0}$ unbounded. Moreover, $\pm i \sqrt{3} / 2 \in \partial R_{0} \cap \partial G_{0} \cap \partial B_{0}$ and $0 \in \partial G_{0} \cap \partial B_{0}$.

Proof. By the above lemma, $R_{0} \cup B_{0} \cup G_{0}$ is disjoint from $[-i \sqrt{3} / 2$, $i \sqrt{3} / 2]$. Because of the dynamical symmetry between the three roots, we have $G\left(R_{0}\right)=G\left(B_{0}\right)=G\left(G_{0}\right) \subset \overline{\mathbb{C}}-G[-i \sqrt{3} / 2, i \sqrt{3} / 2]$. Since $G^{-1}(G([-i \sqrt{3} / 2, i \sqrt{3} / 2]))=G^{-1}([-27 / 4,0])$, each of the three components of $\mathbb{C}-G^{-1}([-27 / 4,0])$ contains one of $B_{0}, G_{0}, R_{0}$. Now one connected component of $\left.\left.G^{-1}(] 0, \infty\right]\right)$ in $\overline{\mathbb{C}}$ is contained in $R_{0}$; the other two must be contained in $B_{0}$ and $G_{0}$ respectively.

For $\lambda=1 / 2$, we have $x_{0}=-1 / 2+\lambda \in A_{\lambda}^{+}$, hence $1 / 2 \in B_{0}$. Similarly, $-1 / 2 \in G_{0}$. We can then specify that $R_{0}$ is contained in the unbounded component of $\mathbb{C}-G^{-1}([-27 / 4,0]), B_{0}$ is contained in the bounded component of $\mathbb{C}-G^{-1}([-27 / 4,0])$ on the right and $G_{0}$ is contained in the bounded com- 
ponent of $\mathbb{C}-G^{-1}([-27 / 4,0])$ on the left. Moreover, since $\pm i \sqrt{3} / 2 \in \partial R_{0}$, via $G$, we get $\pm i \sqrt{3} / 2 \in \partial R_{0} \cap \partial G_{0} \cap \partial B_{0}$.

One can easily check that $0 \in \partial G_{0} \cap \partial B_{0}$ in the following way: take any $\lambda$ with $0<\lambda<1 / 2$. For any $x$ such that $-1 / 2+\lambda<x \leq 0$, we have $-1 / 2+\lambda<N_{\lambda}(x)<x$. Hence $N_{\lambda}^{k}(x)$ tends to a fixed point of $N_{\lambda}$, which has to be $-1 / 2+\lambda$, because we know where the fixed points of $N_{\lambda}$ are (namely, $-1 / 2+\lambda,-1 / 2-\lambda, 1$ and $\infty$ ). This shows $x_{0}=0 \in A_{\lambda}^{+}$and then $\lambda \in B_{0}$. So $] 0,1 / 2\left[\subset B_{0}\right.$. Similarly one can show that $]-1 / 2,0\left[\subset G_{0}\right.$. Now $G^{-1}\left(G\left([-1 / 2,0[))=G^{-1}(]-\infty,-27 / 4\right]\right)=\mathbb{R}-\{ \pm 3 / 2,0\}$, so $\{x \in \mathbb{R} \mid$ $|x|>3 / 2\} \subset R_{0},\{x \in \mathbb{R} \mid-3 / 2<x<0\} \subset G_{0}$ and $\{x \in \mathbb{R} \mid 0<x<3 / 2\}$ $\subset B_{0}$.

Denote by $\Omega$ the closed region in $\mathbb{C}$ bounded by the Jordan curve

$$
[-1 / 2,1 / 2] \cup\left\{-1 / 2+e^{i \theta} \mid 0 \leq \theta \leq \pi / 3\right\} \cup\left\{1 / 2+e^{i \theta} \mid 2 \pi / 3 \leq \theta \leq \pi\right\} .
$$

The region $\Omega$ is a fundamental domain for the Newton's method of cubic polynomials. To be more precise, every $N_{P}$ is conjugate by a Möbius transformation to $N_{\lambda}$ for a $\lambda \in \Omega$, and, for $\lambda$ and $\lambda^{\prime}$ in $\Omega$, the two mappings $N_{\lambda}$ and $N_{\lambda^{\prime}}$ are conjugate iff $\lambda$ and $\lambda^{\prime}$ are on the boundary of $\Omega$ with $\operatorname{Re}\left(\lambda^{\prime}\right)= \pm \operatorname{Re}(\lambda)$

Define $\Psi_{B}:\left(B_{0}, 1 / 2\right) \rightarrow(D, 0)$ such that $\Psi_{B}(\lambda)$ is the location of the co-critical point $\left(=3 \sigma_{3} / 2 \sigma_{2}\right)$ in the Böttcher coordinates of $A_{\lambda}^{+}$. Define $\Psi_{G}:\left(G_{0},-1 / 2\right) \rightarrow(D, 0)$ and $\Psi_{R}:\left(R_{0}, \infty\right) \rightarrow(D, 0)$ similarly.

Lemma 1.3. The map $\Psi_{B}$ (resp. $\left.\Psi_{G}\right)$ is a holomorphic double cover branched at $1 / 2$ (resp. -1/2). One of the two $1 / 2$-rays of $B_{0}$ is $\left.] 0,1 / 2\right]$, one of the 0 -rays of $B_{0}$ is the arc $\left\{-1 / 2+e^{i \theta} \mid 0 \leq \theta \leq \pi / 3\right\}$. Thus $\Omega$ is bounded by 0 - and $1 / 2$-rays of both $B_{0}$ and $G_{0}$.

Pr o of. Again the mapping properties of $\Psi_{B}$ and $\Psi_{G}$ are very similar to Milnor's study in [M1]. We omit the details here. The fact about the $1 / 2$ ray of $B_{0}$ is an easy consequence of the fact that both $N_{\lambda}$ and the Böttcher coordinate are real when $\lambda \in] 0,1 / 2\left[\right.$. The fact about the 0 -ray of $B_{0}$ is proved by proving that the 0-rays of $R_{0}$ stay in the imaginary axis (where the map is real again), and then transferring the result to $B_{0}$ by $G$.

Define $\Lambda$ to be the set of postcritically finite maps in $\Omega$. An interpretation of the Main Theorem in parameter space will be:

Corollary 1.4. For $A$ and $Y$ as in the Main Theorem, the mapping $\mathcal{M}$ satisfies

$$
\mathcal{M}(A) \supset \Lambda \cap\left(C^{-} \cup D\right), \quad \mathcal{M}(Y) \supset \Lambda \cap\left(C^{+} \cup C^{1}\right) .
$$

This corollary will be proved in Section 6.1. 
2. Topological structure of the dynamical plane. The results of this section in the postcritically finite case are mainly due to J. Head. We extend them to the non-postcritically finite case.

2.1. General structure. A cubic polynomial can be written as

$$
P(z)=k\left(z-p_{1}\right)\left(z-p_{2}\right)\left(z-p_{3}\right)
$$

with $p_{1}, p_{2}, p_{3}$ the roots of $P$. Let $N=N_{P}: z \mapsto z-P(z) / P^{\prime}(z)$ be the Newton's method of $P$. The degree of $N$ as a rational map is 3 except when $P$ has multiple roots.

If $P$ has a triple root $p$, its Newton map reduces to a Möbius transformation having $p$ as an attracting fixed point.

If $P$ has a double root $p$ and a simple root $q$, then $N_{P}$ reduces to a degree two rational map having $q$ as a superattracting fixed point and $p$ as an attracting fixed point. The Julia set $J$ of $N_{P}$ is a topological circle, separating the basin $A_{p}$ of $p$ and the basin $A_{q}$ of $q$. The restriction $\left.N\right|_{A_{p}}$ is analytically conjugate to a Blaschke product $z \mapsto z(z-s) /(1-\bar{s} z)(|s|<1)$ on $D_{1}$ while $\left.N\right|_{A_{q}}$ is analytically conjugate to $z \mapsto z^{2}$ on $D_{1}$. Moreover in a neighborhood of the Julia set $N_{P}$ is quasi-conformally conjugate to $z \mapsto z^{2}$.

These degenerate cases are exceptional. In the following we will assume that the roots $p_{1}, p_{2}, p_{3}$ are distinct.

We will work on the Riemann sphere $\overline{\mathbb{C}}$ with the spherical metric. For example $x_{n} \rightarrow \infty$ as $n \rightarrow \infty$ does not mean that $x_{n}$ diverges, but that $x_{n}$ converges to the point $\infty \in \overline{\mathbb{C}}$ with respect to the spherical metric.

We can calculate immediately the fixed points and critical points of $N$ $=N_{P}$ :

Lemma 2.1. 1. The fixed points of $N$ are $p_{1}, p_{2}, p_{3}, \infty$. The eigenvalue of $N$ at $\infty$ is $3 / 2$.

2. The critical points of $N$ are $p_{1}, p_{2}, p_{3}$ and $x_{0}=\left(p_{1}+p_{2}+p_{3}\right) / 3$. We have $P^{\prime \prime}\left(x_{0}\right)=0$, and $p_{1}, p_{2}, p_{3}$ are superattracting fixed points of $N$.

There is a very easy criterion for a rational map to be the Newton's method of a polynomial:

Lemma 2.2 (cf. [He]). Any rational map $F$ of degree d having d distinct superattracting fixed points is conjugate by a Möbius transformation to $N_{P}$ for a polynomial $P$ of degree $d$. Moreover, if $\infty$ is not superattracting for $F$ and $F$ fixes $\infty$, then $F=N_{P}$ for some polynomial $P$ of degree $d$.

In this paper we consider $N_{P}$ as a special kind of rational map, rather than as a root finding algorithm. Moreover we will only treat the cubic case, i.e. $d=3$.

Definition 1. A cubic rational map $N: \overline{\mathbb{C}} \rightarrow \overline{\mathbb{C}}$ is called a Newton map if it has three distinct critical fixed points, denoted by $p_{1}, p_{2}, p_{3}$. Up 
to conjugacy by a Möbius transformation, we may assume that $\infty$ is the unique fixed point of $N$ which is not critical. We denote by $x_{0}$ the free critical point of $N$.

In this subsection we concentrate only on general properties of a Newton map.

We know since Fatou and Julia that the dynamics of a rational map depends on the dynamical behavior of its critical points. In the case that interests us here, three critical points $p_{1}, p_{2}, p_{3}$ are already fixed. The critical point $x_{0}$ is the only one which can have various behaviors. We call $x_{0}$ the free critical point.

Denote by $A_{1}, A_{2}, A_{3}$ the immediate attracting basins of $p_{1}, p_{2}, p_{3}$ respectively. Their topological structure is extremely simple, according to a result of F. Przytycki:

Lemma 2.3 ([Prz $])$. Each $A_{i}$ is simply connected and $\infty \in \partial A_{i}, i=$ $1,2,3$.

The idea of the proof is to show that if $A_{i}$ is not simply connected then there are at least two fixed points of $N$ on the boundary. The proof uses only classical results, such as Montel's theorem. We can also construct a proof using the Poincaré metric of $A_{i}$, following Douady-Hubbard's method for landing points of rays (cf. [DH1], part I, pp. 70-71).

Combining the Böttcher theorem and a result of Douady-Hubbard we can give an analytic model for the dynamics of $\left.N\right|_{A_{i}}$ whenever $x_{0} \notin A_{i}$.

Lemma 2.4. Set $D=\{z \in \mathbb{C}|| z \mid<1\}$. Fix $i \in\{1,2,3\}$ such that $x_{0} \neq p_{i}$.

1 (Böttcher). There is a unique holomorphic mapping $\varphi_{i}: D-X_{i} \rightarrow A_{i}$ such that $\varphi_{i}(0)=p_{i}, \varphi_{i}^{\prime}(0) \neq 0$, and the following diagram commutes:

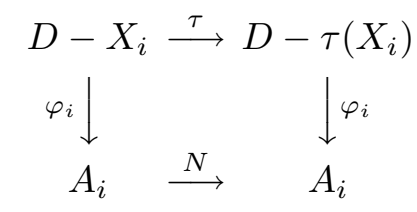

where $\tau$ denotes $z \mapsto z^{2}, X_{i}=\emptyset$ and $\varphi_{i}(D)=A_{i}$ if $x_{0} \notin A_{i}$, or else $X_{i}=\bigcup_{n \geq 0} \tau^{-n}\left\{r e^{2 \pi i \theta_{0}} \mid r_{0} \leq r \leq 1\right\}$ with $r_{0}, \theta_{0}$ the values for which $\varphi_{i}\left(r_{0} e^{2 \pi i\left(\theta_{0}+1 / 2\right)}\right)$ is the co-critical point.

2 (Douady-Hubbard). The mapping $\varphi_{i}: D-X_{i} \rightarrow A_{i}$ has radial limits at each point of $\partial D-X_{i}$ with rational angle (counted in turns). That is, for $t \in \mathbb{Q} / \mathbb{Z}$ and $e^{2 \pi i t} \notin X_{i}$, the limit $\lim _{r}{ }_{1} \varphi_{i}\left(r e^{2 \pi i t}\right)$ exists, and is contained in $\partial A_{i}$. In particular, if $\theta_{0} \neq 0,1 / 2$, we have

3. $\varphi_{i}(1)=\lim _{r}{ }_{1} \varphi_{i}(r)=\infty$, and $\lim _{r}{ }_{1} \varphi_{i}\left(r e^{\pi i}\right)$ exists and is a preimage of $\infty$. 
The existence and unicity of $\varphi_{i}$ in a neighborhood of 0 can be found for example in [M2]. The rest of assertion 1 is done by a pull-back argument. A proof of assertion 2 is contained in [DH1], part I, pp. 70-71. Point 3 follows from point 2 .

Assume that the free critical point $x_{0}$ is contained in one of $A_{i}$, say $A_{1}$. A typical example for this is $N_{Q}$ for $Q(z)=z^{3}-z$. We have $x_{0}=p_{1}=0$ and $p_{2,3}= \pm 1$. The mapping $N_{Q}$ is analytically conjugate to $\mathbf{f}: z \mapsto z^{3}+(3 / 2) z$ in the entire Riemann sphere. In general $x_{0} \in A_{1}$ is disjoint from $p_{1}$. In this case, the restriction $\left.N\right|_{A_{1}}$ is analytically conjugate to a Blaschke product $z \mapsto \lambda z^{2}(z-s) /(1-\bar{s} z)(|\lambda|=1,|s|<1)$ on $D_{1}$ (since $N: A_{1} \rightarrow A_{1}$ is a proper mapping of degree 3 with one simple critical point fixed), while $\left.N\right|_{J_{N}}$ is quasi-conformally conjugate to $\left.\mathbf{f}\right|_{J_{\mathbf{f}}}$ (in fact, $N$ is a cubic polynomial-like mapping in the complement of a disc containing $p_{1}$ and $x_{0}$; see [D3] or [DH3]).

Fix $i$ such that $x_{0} \notin A_{i}$. Since $p_{i}$ is a simple critical point of $N$, the mapping $N: A_{i} \rightarrow A_{i}$ is a double covering ramified at $p_{i}$. Moreover, $A_{i}$ is a connected component of $N^{-1}\left(A_{i}\right)\left(\partial A_{i} \subset J_{N}\right)$. But $N$ is of degree three. There must be another connected component $W_{i}$ of $N^{-1}\left(A_{i}\right)$, with $N: W_{i} \rightarrow A_{i}$ a homeomorphism. We can lift $\varphi_{i}$ by $N$ and claim that there is a unique conformal mapping $\psi_{i}: D \rightarrow W_{i}$ such that $N \circ \psi_{i}(z)=\varphi_{i}(z)$.

Similarly $N^{-1}(\infty)$ contains three points, which we denote by $\xi_{1}, \xi_{2}, \infty$. Since $\infty$ is not a critical point, we have $\xi_{1} \neq \infty$ and $\xi_{2} \neq \infty$. The case $\xi_{1}=\xi_{2}$ is quite exceptional, because in this case $\xi_{1}=\xi_{2}=x_{0}$, and $N$ is conjugate by a Möbius transformation to the Newton's method $N_{Q}$ for $Q: z \mapsto z^{3}-1$. Its dynamics is simple and has been well studied in the literature. (This is Cayley's original problem and is well studied. See for example [HP].) We will give some description of its dynamics in the following as well.

For $t \in \mathbb{T}$, we define the $t$-ray of $A_{i}$ and of $W_{i}$ to be

$$
a_{i}(t)=\left\{\varphi_{i}\left(r e^{2 \pi i t}\right) \mid 0 \leq r<1\right\}, \quad w_{i}(t)=\left\{\psi_{i}\left(r e^{2 \pi i t}\right) \mid 0 \leq r<1\right\} .
$$

If $a_{i}(t)$ (resp. $\left.w_{i}(t)\right)$ lands at a point of $\partial A_{i}$ (resp. $\left.\partial W_{i}\right)$, we denote the landing point by $\iota_{i}(t)\left(\operatorname{resp} . \sigma_{i}(t)\right)$. In other words, $\iota_{i}(t)=\varphi_{i}\left(e^{2 \pi i t}\right)\left(\sigma_{i}(t)=\right.$ $\left.\psi_{i}\left(e^{2 \pi i t}\right)\right)$. Moreover, set

$$
\begin{gathered}
\bar{a}_{i}(t)=\overline{a_{i}(t)}=\left\{\varphi_{i}\left(r e^{2 \pi i t}\right) \mid 0 \leq r \leq 1\right\}=a_{i}(t) \cup\left\{\iota_{i}(t)\right\}, \\
\bar{w}_{i}(t)=\overline{w_{i}(t)}=\left\{\psi_{i}\left(r e^{2 \pi i t}\right) \mid 0 \leq r \leq 1\right\}=w_{i}(t) \cup\left\{\sigma_{i}(t)\right\} .
\end{gathered}
$$

The conjugacy in Lemma 2.4 ensures that the rays are preserved by dynamics. More precisely, $N\left(a_{i}(t)\right)=a_{i}(2 t), N\left(w_{i}(t)\right)=a_{i}(t)$ and $N^{-1}\left(a_{i}(t)\right)=$ $a_{i}(t / 2) \cup a_{i}(t / 2+1 / 2) \cup w_{i}(t)$. Moreover, $\iota_{i}(t)$ exists if and only if one of $\iota_{i}(t / 2), \iota_{i}(t / 2+1 / 2), \sigma_{i}(t)$ exists.

In case $x_{0} \in A_{i}$, set $W_{i}=A_{i}$. For $e^{2 \pi i t} \notin X_{i}$, we can define $a_{i}(t)$ similarly, and we define $\iota_{i}(t)$ as before if $a_{i}(t)$ lands. 
Assume that either $x_{0} \notin A_{1} \cup A_{2} \cup A_{3}$, or $x_{0} \in A_{i}$ for some $i$ but the co-critical point is not on the 0 - or $1 / 2$-ray of $A_{i}$ (i.e. $\theta_{0} \neq 0,1 / 2$ in Lemma 2.4.1. So the 0 - and 1/2-rays are well defined and land in each of $A_{1}, A_{2}, A_{3}$. Assume that the 0 -rays $a_{1}(0), a_{2}(0), a_{3}(0)$ are connected at $\infty$ in counterclockwise order. The first striking result of J. Head is ([He], Proposition 3.2.1):

LeMma 2.5. In case $\xi_{1} \neq \xi_{2}$, one can renumber $p_{1}, p_{2}, p_{3}$ so that

$$
\begin{aligned}
& \xi_{1}=\iota_{1}(1 / 2)=\iota_{2}(1 / 2)=\sigma_{3}(1) \in \partial A_{1} \cap \partial A_{2} \cap \partial W_{3}, \\
& \xi_{2}=\sigma_{1}(1)=\sigma_{2}(1)=\iota_{3}(1 / 2) \in \partial W_{1} \cap \partial W_{2} \cap \partial A_{3} .
\end{aligned}
$$

Moreover, $x_{0} \notin A_{3}$.

Proof. Note that $\iota_{i}(1 / 2), \sigma_{i}(1) \in N^{-1}(\infty), i=1,2,3$. Since $N$ is locally injective at $\infty$, we must have $\iota_{i}(1 / 2), \sigma_{i}(1) \in\left\{\xi_{1}, \xi_{2}\right\}, i=1,2,3$. Two of $\iota_{1}(1 / 2), \iota_{2}(1 / 2), \iota_{3}(1 / 2)$ must coincide. Assume $\xi_{1}=\iota_{1}(1 / 2)=\iota_{2}(1 / 2)$. The rest is proved essentially by looking at the cyclic order of components attached at $\xi_{1}$, which should be preserved by $N$. See [He]. The cyclic order at $\xi_{1}$ shows also $W_{3} \neq A_{3}$. So $x_{0} \notin A_{3}$.

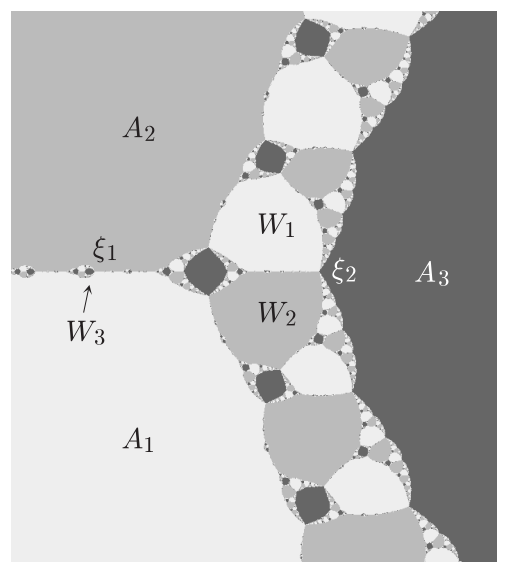

Fig. $3^{\prime} . A_{i}, W_{i}$ and $\xi_{1}, \xi_{2}$

An immediate consequence of this lemma is:

Proposition 2.6. The Julia set $J_{N}$ for a cubic Newton map $N$ is connected.

R e m ark. According to a result of M. Shishikura ([Sh1]), the Julia set of $N_{P}$ for any polynomial $P$ is always connected. His proof uses essentially the technique of holomorphic surgery. In the degree three case, however, we can give a more elementary argument, using ideas of H.-G. Meier ([Me]), who got the same result under some extra assumptions. 
P r o of (of Proposition 2.6). Note first that any simply connected open subset $U$ of $\overline{\mathbb{C}}$ has a connected boundary (for a conformal mapping $u: D \rightarrow$ $U$, the boundary $\partial U$ coincides with $\bigcap_{n} \overline{u(\{1-1 / n<|z|<1\}))}$.

We only need to examine the case $p_{1}, p_{2}, p_{3}$ distinct and $x_{0} \notin A_{1} \cup A_{2} \cup A_{3}$. By the above lemma the set $N^{-1}\left(\partial A_{1} \cup \partial A_{2}\right)$ is connected and contains $\partial A_{1} \cup \partial A_{2}$. We first prove that $N^{-k}\left(\partial A_{1} \cup \partial A_{2}\right)$ is connected and contains $N^{-(k-1)}\left(\partial A_{1} \cup \partial A_{2}\right)$ for every integer $k$.

Let $U$ be a connected component of $\overline{\mathbb{C}}-J_{N}$ such that $N^{k}(U)=A_{i}$ for some $k \in \mathbb{N}$ and some $i \in\{1,2\}$. Denote by $k(U)$ the minimal integer $k$ satisfying the above equality. Since $N^{k(U)}: U \rightarrow A_{i}$ is ramified at at most one point, $U$ is simply connected and $\partial U$ is connected. It is quite easy to see that $\partial N^{-1}(U)=N^{-1}(\partial U)$. Hence

$$
N^{-k}\left(\partial A_{1} \cup \partial A_{2}\right)=\partial N^{-k}\left(A_{1} \cup A_{2}\right)=\partial N^{-k}\left(\overline{A_{1} \cup A_{2}}\right) .
$$

By induction on $k$ we can prove that for any $k \in \mathbb{N}$ and any $U$ with $k(U)=k$, there is a preimage component $V$ of $A_{1}, A_{2}$ such that $k(V)<k(U)$ and $\bar{U} \cap \bar{V} \neq \emptyset$. So $\partial U \cup \partial V$ is connected.

We conclude that $\bigcup_{k=0}^{K} N^{-k}\left(\partial A_{1} \cup \partial A_{2}\right)$ is connected for all $K$. Thus

$$
\operatorname{closure}\left(\bigcup_{k \geq 0} N^{-k}\left(\partial A_{1} \cup \partial A_{2}\right)\right)
$$

is also connected.

The set (1) is clearly a subset of $J_{N}$. Just as $J_{N}$, the set (1) is a completely invariant compact set of $N$ and contains more than two points. Since $J_{N}$ is the unique minimal compact set with these properties (cf. [B]), we conclude that $J_{N}$ coincides with (1). It is thus connected.

Again from Lemma 2.5, we can get more information about relations between $\partial A_{i}, \partial W_{i}, i=1,2,3$.

Convention. Under the assumption that either $x_{0} \notin A_{1} \cup A_{2} \cup A_{3}$ or $x_{0} \in A_{i}$ for some $i$ but not in the 0 - and 1/2-ray of $A_{i}$, we always number $p_{1}, p_{2}, p_{3}$ in the way described in Lemma 2.5 , i.e. so that the 0 -rays of $A_{1}, A_{2}, A_{3}$ are connected at $\infty$ in counterclockwise order and $\xi_{1}=\iota_{1}(1 / 2)=$ $\iota_{2}(1 / 2)$. We call this the dynamical numbering of the three fixed points.

Lemma 2.7. Suppose $x_{0} \notin A_{1} \cup A_{2} \cup A_{3}$.

$1\left([\mathrm{He}]\right.$, Proposition 3.2.2). $\iota_{1}\left(-1 / 2^{n}\right)=\iota_{2}\left(1 / 2^{n}\right) \in \partial A_{1} \cap \partial A_{2}, n \in \mathbb{N}^{+}$.

2. There is an integer $l \geq 1$ such that for $n=0,1, \ldots, l-1$,

$$
\iota_{1}\left(1 / 2^{n}\right)=\iota_{2}\left(-1 / 2^{n}\right) \in \partial A_{1} \cap \partial A_{2}
$$

but $\iota_{1}\left(1 / 2^{l}\right)=\sigma_{2}\left(-1 / 2^{l-1}\right) \in \partial A_{1} \cap \partial W_{2}$ and $\iota_{2}\left(-1 / 2^{l}\right)=\sigma_{1}\left(1 / 2^{l-1}\right) \in$ $\partial W_{1} \cap \partial A_{2}$. And the last two points coincide if and only if one of them is equal to $x_{0}$. 
3. For the free critical point $x_{0}$, either $x_{0}=\iota_{1}\left(1 / 2^{l}\right)$, or it is separated from $\infty$ by the following closed curve:

$$
\begin{aligned}
\eta= & \bar{a}_{1}\left(1 / 2^{l-1}\right) \cup \bar{a}_{2}\left(-1 / 2^{l-1}\right) \cup \bar{a}_{1}\left(1 / 2^{l}\right) \cup \bar{a}_{2}\left(-1 / 2^{l}\right) \\
& \cup \bar{w}_{1}\left(1 / 2^{l-1}\right) \cup \bar{w}_{2}\left(-1 / 2^{l-1}\right) \cup \bar{w}_{1}\left(1 / 2^{l-2}\right) \cup \bar{w}_{2}\left(-1 / 2^{l-2}\right) .
\end{aligned}
$$

(Recall that for $t$ rational, $\left.\bar{a}_{i}(t)=a_{i}(t) \cup\left\{\iota_{i}(t)\right\}\right)$.

4. The impression of the prime end associated with $a_{i}(0)$ is $\infty, i=1,2,3$, and $\lim _{n \rightarrow \infty} \iota_{1}\left(-1 / 2^{n}\right)=\infty$.

$5\left([\mathrm{He}]\right.$, Proposition 3.2.2). $\partial A_{3} \cap\left(\partial A_{1} \cup \partial A_{2}\right)=\{\infty\}$.

Proof. Point 1 is proved in [He]. The idea is to look at the position of points in $N^{-1}\left(\xi_{1}\right)$ relative to the Jordan curve $\bar{a}_{1}(0) \cup \bar{a}_{2}(0) \cup \bar{a}_{2}(1 / 2) \cup$ $\bar{a}_{1}(1 / 2)$.

2. Suppose that for all $n \in \mathbb{N}, \iota_{1}\left(1 / 2^{n}\right)=\iota_{2}\left(-1 / 2^{n}\right)$. Choose $s_{0}<0$ and set $s_{n}=s_{0} / 2^{n}$. For $n \geq 2$, define $\Gamma_{n}$ to be the following Jordan curve, made by parts of rays and parts of equi-potentials of $A_{1}, A_{2}$ :

$$
\begin{aligned}
\Gamma_{n}= & \left\{\varphi_{1}\left(e^{s+2 \pi i / 2^{n}}\right) \mid s_{n} \leq s \leq 0\right\} \cup\left\{\varphi_{2}\left(e^{s-2 \pi i / 2^{n}}\right) \mid s_{n} \leq s \leq 0\right\} \\
& \cup\left\{\varphi_{2}\left(e^{s_{n}+2 \pi i \theta}\right) \mid \theta \in\left[-1 / 2^{n}, 1\right] \cup\left[0,1 / 2^{n}\right]\right\} \\
& \cup\left\{\varphi_{2}\left(e^{s+2 \pi i / 2^{n}}\right) \mid s_{n} \leq s \leq 0\right\} \cup\left\{\varphi_{1}\left(e^{s-2 \pi i / 2^{n}}\right) \mid s_{n} \leq s \leq 0\right\} \\
& \cup\left\{\varphi_{1}\left(e^{s_{n}+2 \pi i \theta}\right) \mid \theta \in\left[-1 / 2^{n}, 1\right] \cup\left[0,1 / 2^{n}\right]\right\} .
\end{aligned}
$$

Denote by $B_{n}$ the annulus bounded by the two loops $\Gamma_{n}, \Gamma_{n+1}$. Then $B_{2}, B_{3}, \ldots$ form a nested sequence of annuli with $\bar{A}_{3}$ contained in the end of the nest. It is easy to check that $N\left(B_{n}\right)=B_{n-1}$, and $N: B_{n} \rightarrow B_{n-1}$ is a holomorphic homeomorphism. Hence all annuli $B_{n}$ have the same complex modulus. By a classical result of complex analysis, there is only one point at the end of the nest. This gives a contradiction.

3. The curve $\eta$ is a connected component of $N^{-1}\left(\eta^{\prime}\right)$ where

$$
\eta^{\prime}=\bar{a}_{1}\left(1 / 2^{l-1}\right) \cup \bar{a}_{2}\left(-1 / 2^{l-1}\right) \cup \bar{a}_{1}\left(1 / 2^{l-2}\right) \cup \bar{a}_{2}\left(-1 / 2^{l-2}\right) .
$$

Looking at the preimages of components of $\overline{\mathbb{C}}-\eta^{\prime}$, we can see that $N$ maps the connected component $V$ of $\overline{\mathbb{C}}-\eta$ with $\infty \notin V$ onto the connected component $V^{\prime}$ of $\overline{\mathbb{C}}-\eta^{\prime}$ with $\infty \notin V^{\prime}$, and $\operatorname{deg}\left(N: \partial V \rightarrow \partial V^{\prime}\right)=2$. So $x_{0} \in V$ and $N: V \rightarrow V^{\prime}$ is a double covering ramified at $x_{0}$.

4. This will follow essentially from the same argument as the proof of point 2. Define

$$
\begin{aligned}
g= & \bar{a}_{1}(1 / 2) \cup \bar{a}_{2}(1 / 2) \cup \bar{a}_{1}\left(1 / 2^{l}\right) \cup \bar{a}_{2}\left(-1 / 2^{l}\right) \\
& \cup \bar{w}_{2}\left(-1 / 2^{l-1}\right) \cup \bar{w}_{2}(0) \cup \bar{a}_{3}(1 / 2) \cup \bar{w}_{1}(0) \cup \bar{w}_{1}\left(1 / 2^{l-1}\right)
\end{aligned}
$$

(see Figure $4^{\prime}$ ). We first construct a new graph in the following way: Take a small neighborhood $U$ of $p_{1}$ bounded by an equi-potential. Replace $g \cap U$ by the connected component of $\partial U-g$ intersecting $a_{1}(0)$. Make similar 
modifications in neighborhoods of $p_{2}$ and $p_{3}$. We get our graph $g_{0}$. The complement of $g_{0}$ has a component $S$ containing $\infty$ but no critical points. Now taking inductively the connected component of $N^{-1}\left(g_{k}\right)$ contained in $S$, we can construct a nested sequence of annuli having the same modulus and containing $\infty$ in the end of the nest. Hence the end consists of only one point $\infty$. For each $i=1,2,3$, we have also constructed a sequence of cross cuts cutting $a_{i}(0)$, and converging to $\infty$.

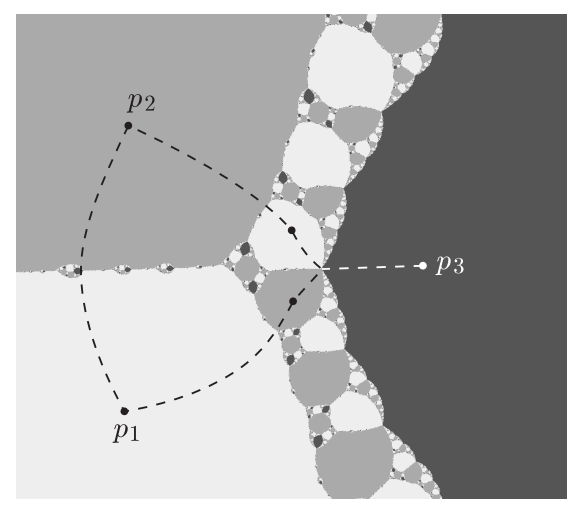

Fig. $4^{\prime}$. The graph $g$

5. In the first annulus constructed in assertion 4 one can find an arc which is a preimage of

$$
\bar{w}_{1}(1 / 2) \cup \bar{w}_{1}\left(1 / 2^{l-1}\right) \cup \bar{a}_{2}\left(-1 / 2^{l}\right),
$$

separating $\partial A_{1}$ and $\partial A_{3}$, and another arc separating $\partial A_{2}$ and $\partial A_{3}$.

The integer $l$ guarantees that for any $t \in \mathbb{T}, t<1 / 2^{l}, \bar{a}_{1}(t) \cap \bar{A}_{2}=\emptyset$. More precisely, the graph

$$
\begin{aligned}
\bar{a}_{1}(0) \cup \bar{a}_{1}\left(1 / 2^{l}\right) \cup & \bar{w}_{2}\left(-1 / 2^{l-1}\right) \cup \bar{w}_{2}(0) \cup \bar{w}_{1}(0) \\
& \cup \bar{w}_{1}\left(1 / 2^{l-1}\right) \cup \bar{a}_{2}\left(-1 / 2^{l}\right) \cup \bar{a}_{2}(0) \cup \bar{a}_{3}(0) \cup \bar{a}_{3}(1 / 2)
\end{aligned}
$$

separates $\overline{\mathbb{C}}$ into 4 or 3 commected components. In any case denote by $V$ the connected component containing $\iota_{1}(1 / 2)$. Then

Corollary 2.8. $\partial A_{1} \cap \partial A_{2} \subset \bar{V}$.

In general we do not know if $a_{i}(t)$ lands at a point of $\partial A_{i}$ for all $t$. But if the Julia set $J_{N}$ is locally connected, then according to the Carathéodory theorem (cf. [DH1], part I, p. 11) $\varphi_{i}$ (resp. $\psi_{i}$ ) extends continuously to $\partial D$. In this case the dynamics of any point $z \in \partial A_{i}$ is completely determined by the angle $t$ such that $a_{i}(t)$ lands at $z$. A priori it might happen that $a_{i}(t)$ and $a_{i}\left(t^{\prime}\right)$ land at the same point. But since we have three basins, the 
extension of $\varphi_{i}$ is in fact a homeomorphism, as we are going to see in the next proposition. The proof is due to M. Shishikura.

Proposition 2.9. Assume that $x_{0} \notin A_{1} \cup A_{2} \cup A_{3}$ and $J_{N}$ is locally connected. Then for $i=1,2,3$, the mappings $\varphi_{i}: D \rightarrow A_{i}$ and $\psi_{i}: D \rightarrow W_{i}$ extend homeomorphically to $\bar{D}$, with $\varphi_{i}(\partial D)=\partial A_{i}$ and $\psi_{i}(\partial D)=\partial W_{i}$. As a consequence, the sets $\partial A_{i}$ and $\partial W_{i}$ are Jordan curves.

Proof. We only need to study $\varphi_{i}, i=1,2,3$. By the Carathéodory theorem, every ray of $A_{i}$ lands at a point of $\partial A_{i}$.

Recall that $\infty \neq \xi_{1}$ and $\infty \neq \xi_{2}$. First of all we show that the point $\infty$ accepts no ray other than the 0 -ray of $A_{i}, i=1,2,3$. Suppose that $a_{i}(\theta)$ lands at $\infty$ with $0<\theta<1 / 2$. Then there is $n \in \mathbb{N}$ such that $0<$ $2^{n} \theta<1 / 2<2^{n+1} \theta<1$. The graph $\bar{a}_{i}(0) \cup \bar{a}_{i}\left(2^{n} \theta\right) \cup \bar{a}_{i}\left(2^{n+1} \theta\right)$ cuts $\overline{\mathbb{C}}$ into three components, each of them containing exactly one of $a_{i}(1 / 2)-\left\{p_{i}\right\}$, $a_{i}\left(2^{n+1} \theta-1 / 2\right)-\left\{p_{i}\right\}$ and $a_{i}\left(2^{n} \theta+1 / 2\right)-\left\{p_{i}\right\}$. But the last three rays should also land at the same point (which is either $\xi_{1}$ or $\xi_{2}$ ). This is impossible because the rays are disjoint, except at their extremities.

Next let us show that each $\xi_{1}, \xi_{2}$ accepts at most one ray from $A_{1}, A_{2}, A_{3}$. If $\xi_{1} \neq \xi_{2}$, the mapping $N$ is injective in a neighborhood of $\xi_{1}$ (resp. $\xi_{2}$ ). It then sends injectively each ray of $A_{i}(i=\{1,2,3\})$ landing at $\xi_{1}$ (resp. $\left.\xi_{2}\right)$ to a ray of $A_{i}$ landing at $\infty$. But there is only one such ray, so there is at most one ray from $A_{i}$ landing at $\xi_{1}$ (resp. $\xi_{2}$ ). If $\xi_{1}=\xi_{2}$, then $N$ is of degree 2 in a neighborhood of $\xi_{1}$. Since $a_{i}(1 / 2)$ and $w_{i}(0)$ both land at $\xi_{1}$, and $N\left(a_{i}(1 / 2)\right)=N\left(w_{i}(0)\right)=a_{i}(0)$, there is no other ray from $A_{i}$ landing at $\xi_{1}, i=1,2,3$.

The union $\bar{a}_{1}(0) \cup \bar{a}_{2}(0) \cup \bar{a}_{1}(1 / 2) \cup \bar{a}_{2}(1 / 2)$ forms a Jordan curve $C$. For $\theta \neq \theta^{\prime}$, there is an $n \in \mathbb{N}$ such that $\left.\left.2^{n} \theta \in\right] 0,1 / 2\right]$ and $\left.\left.2^{n} \theta^{\prime} \in\right] 1 / 2,1\right]$. If one of them is 0 or $1 / 2$, we know already that $a_{1}\left(2^{n} \theta\right)$ and $a_{1}\left(2^{n} \theta^{\prime}\right)$ land at different points. If neither of them is 0 or $1 / 2$, then $\bar{a}_{1}\left(2^{n} \theta\right)-\left\{p_{1}\right\}$ and $\bar{a}_{1}\left(2^{n} \theta^{\prime}\right)-\left\{p_{1}\right\}$ are contained in different components of $\overline{\mathbb{C}}-C$. So $a_{1}\left(2^{n} \theta\right)$ and $a_{1}\left(2^{n} \theta^{\prime}\right)$ land at different points. As a consequence, $a_{1}(\theta)$ and $a_{1}\left(\theta^{\prime}\right)$ land at different points.

This shows that $\varphi_{1}$ extends homeomorphically to the boundary of $D$. Clearly the same is true of $\varphi_{2}$. Concerning $\varphi_{3}$, we need to take another Jordan curve $C$, and then proceed in the same way. If $\xi_{1}=\xi_{2}$, we can take $C=\bar{a}_{1}(0) \cup \bar{a}_{3}(0) \cup \bar{a}_{1}(1 / 2) \cup \bar{a}_{3}(1 / 2)$. If $\xi_{1} \neq \xi_{2}$, let $l$ be the integer of Lemma 2.7.2, and take

$$
C=\bar{a}_{1}(0) \cup \bar{a}_{3}(0) \cup \bar{a}_{1}\left(1 / 2^{l}\right) \cup \bar{a}_{3}(1 / 2) \cup \bar{w}_{2}(0) \cup \bar{w}_{2}\left(-1 / 2^{l-1}\right) .
$$

We are now ready to determine the set $\partial A_{1} \cap \partial A_{2}$. Define

$$
T_{N}=\left\{t \in \mathbb{T} \mid \iota_{1}(t)=\iota_{2}(-t)\right\} .
$$


Corollary 2.10. Assume that $J_{N}$ is locally connected, and that $x_{0}$ is not contained in $A_{1} \cup A_{2} \cup A_{3}$. The mapping $\iota_{1}$ gives rise to a homeomorphism from $T_{N}$ to $\partial A_{1} \cap \partial A_{2}$ and conjugates $\tau$ to $N$.

P r o of. Clearly $\iota_{1}\left(T_{N}\right) \subset \partial A_{1} \cap \partial A_{2}$. The mapping $\iota_{1}$ is injective by the above proposition.

Now let $z \in \partial A_{1} \cap \partial A_{2}$. Again by the above theorem, there are two angles $t, s \in \mathbb{T}$ such that $z=\iota_{1}(t)=\iota_{2}(-s)$. Denote by $C$ the Jordan curve $\bar{a}_{1}(0) \cup \bar{a}_{2}(0) \cup \bar{a}_{1}(1 / 2) \cup \bar{a}_{2}(1 / 2)$. The binary expansion of $t$ (resp. $\left.-s\right)$ is determined by the position of $a_{1}\left(2^{n} t\right)-\{0\}$ (resp. $a_{2}\left(-2^{n} s\right)-\{0\}$ ) with respect to $\overline{\mathbb{C}}-C$. Since $a_{1}(t)$ and $a_{2}(-s)$ land at the same point, the angles $t$ and $-s$ have opposite expansion. Thus $t=s$.

In the next subsection we will see that $T_{N}$ is determined by its minimal element.

To end this subsection, we state a relative result of F. von Haeseler about the Jordan curve property of the boundary of an attracting basin (Theorem 2.14 of $[\mathrm{Ha}])$.

Proposition 2.11 ([Ha]). Let $U \subset S^{2}$ open and simply connected. Suppose that $F: \bar{U} \rightarrow \bar{U}$ and $H: \bar{D} \rightarrow \bar{U}(\bar{D}=\{|z| \leq 1\})$ are continuous mappings with the following properties:

1. $\#\left\{F^{-1}(x)\right\} \leq 2$ for each $x \in \partial U$.

2. $H: D \rightarrow U$ is a homeomorphism.

3. $F(H(z))=H\left(z^{2}\right)$ for each $z \in \bar{D}$.

4. F extends to an injective map in a neighborhood of $H(1)$ in $S^{2}$.

Then $H: \partial D \rightarrow \partial U$ is a homeomorphism if and only if there exists an open connected non-empty set $V \subset S^{2}-U$ such that $H(1) \in \partial V$.

Condition 1 is often difficult to check. For example Shishikura's proof of Proposition 2.9 does not use this theorem because of this difficulty. Condition 4 is not stated in [Ha] but is used implicitly in the proof, and a counterexample of Shishikura shows that it cannot be omitted. But in the case where $F$ is a branched covering of $S^{2}$ this condition is always satisfied.

2.2. A combinatorial study. Now we are going to study a closed subset $\mathcal{S}$ of $\mathbb{T}=\mathbb{R} / \mathbb{Z}$. Later we will see reasons for there being a one-to-one correspondence between the connected components of $\mathbb{C}-\bar{B}_{0} \cup \bar{G}_{0}$ in $\Omega$ and the connected components of $(\mathbb{T}-\mathcal{S})-] 1 / 2,1[$.

Definition. Let $\mathbb{T}$ be the quotient space $[0,1] /\{0 \sim 1\}$. The order in $\mathbb{T}$ is the quotient of the order in the half-open interval $] 0,1]$. An interval of $\mathbb{T}$ is the quotient of an interval in $] 0,1]$. Set $\tau: \mathbb{T} \rightarrow \mathbb{T}, t \mapsto 2 t(\bmod 1)$. For $a \in \mathbb{T}$, define

$$
G_{a}=\left\{t \in \mathbb{T} \mid \tau^{n}(t) \in[a, 1], n=0,1,2, \ldots\right\},
$$




$$
\mathcal{S}=\left\{a \in \mathbb{T} \mid a \in G_{a}\right\}=\left\{a \in \mathbb{T} \mid \tau^{n}(a) \in[a, 1], n=0,1,2, \ldots\right\} .
$$

Note that for $a \in] 1 / 2,1], G_{a}=\{1\}$.

The mapping $\tau$ has the following properties: For any interval $I$ with $1 / 2 \notin I, \tau(I)$ is again an interval and $\tau: I \rightarrow \tau(I)$ is a homeomorphism. Conversely, for any interval $I, \tau^{-1}(I)$ consists of two intervals $I_{1}$ and $I_{2}$ with $\left.\left.\left.\left.I_{1} \subset\right] 0,1 / 2\right], I_{2} \subset\right] 1 / 2,1\right]$ and $\tau: I_{1}, I_{2} \rightarrow I$ are homeomorphisms.

Lemma 2.12. Let $a \in] 0,1 / 2]$. The set $G_{a}$ is closed, forward invariant $\left(\tau\left(G_{a}\right)=G_{a}\right)$, without interior and with 1 as an accumulation point. Any connected component I of $\mathbb{T}-G_{a}$ is an open interval. Denote by $I_{0}$ the one containing $] 0, a\left[\right.$. For any $I \neq I_{0}, \tau(I)$ is again a connected component of $\mathbb{T}-G_{a}$.

Proof. The set $\mathbb{T}-G_{a}=\bigcup_{n=0}^{\infty} \tau^{-n}(] 0, a[)$ is open. We have $\tau\left(G_{a}\right) \subset$ $G_{a}$. On the other hand, for any $t \in G_{a}$, the point $1 / 2+t / 2$ is in $G_{a} \cap \tau^{-1}\left(G_{a}\right)$. Hence $t \in \tau\left(G_{a}\right)$.

Suppose that the interior of $G_{a}$ contains an interval $] s, t[$. There are minimal integers $k, p$ such that $s \leq p / 2^{k}<t$. Then $\tau^{k}$ is injective in ]$p / 2^{k}, t\left[\right.$ and $\left.\tau^{k}(] p / 2^{k}, t[)=\right] 0, \tau^{k} t\left[\subset G_{a}\right.$. But by definition $\left.G_{a} \cap\right] 0, a[=\emptyset$, a contradiction.

We have $\left(2^{n}-1\right) / 2^{n} \in G_{a}$ for $n \in \mathbb{N}$ and $\left(2^{n}-1\right) / 2^{n} \rightarrow 1$ as $n \rightarrow \infty$.

Let $I$ be a connected component of $\mathbb{T}-G_{a}$. Since $1 \notin I, I$ is an open interval. Assume $I \neq I_{0}$ and $\left.I=\right] t, t^{\prime}$. We have $1 / 2 \notin I$ since $1 / 2 \in G_{a}$. So $\tau(I)$ is again an interval, the interval $] \tau(t), \tau\left(t^{\prime}\right)[$. We want to show ]$\tau(t), \tau\left(t^{\prime}\right)\left[\cap G_{a}=\emptyset\right.$. If not, there is $s \in I$ such that $\tau(s) \in G_{a}$. Since $s \geq t \geq a, s \in G_{a}$ by definition. This contradicts the choice of $I$.

LEMma 2.13. Let $G \subset \mathbb{T}$ be a closed set satisfying:

(a) $\tau(G) \subset G$;

(b) $\left.I_{0}=\right] 0, \alpha[$ is a connected component of $\mathbb{T}-G$;

(c) 1 is an accumulation point; and

(d) for each connected component $I \neq I_{0}$ of $\mathbb{T}-G, \tau(I)$ is a connected component of $\mathbb{T}-G$.

Then $G=G_{\alpha}$ for some $\alpha \in \mathcal{S}$.

Proof. For any $t \in G$, we have $t \geq \alpha$ and $\tau^{n}(t) \in G$. So $G \subset G_{\alpha}$, and $\alpha \in \mathcal{S}$.

Let us now prove $\mathbb{T}-G \subset \mathbb{T}-G_{\alpha}$. First, $I_{0} \subset \mathbb{T}-G_{\alpha}$. Let $I$ be a connected component of $\mathbb{T}-G$. We have $1 \notin I$. So $I=] t, t^{\prime}[$ is an interval. We also have $1 / 2 \notin I$ for otherwise $\tau(I)$ would be a connected component of $\mathbb{T}-G$ containing 1. There are minimal integers $k$ and $p$ such that $t \leq p / 2^{k} \leq t^{\prime}$. Then $\tau^{k}: I \rightarrow \tau^{k}(I)$ is injective. Since $\tau^{j}(t) \neq 0$ for $j=0,1, \ldots, k-1$, $\tau^{j}(I) \neq I_{0}$. So $\tau^{k}(I)$ is a connected component of $\mathbb{T}-G$, with 1 in the 
extremity. Since 1 is an accumulation point, we have $\tau^{k}(I)=I_{0}$. In other words, for any $t \in I, \tau^{k}(t)<\alpha$. That is, $I \subset \mathbb{T}-G_{\alpha}$.

COROllary 2.14. Let $s \in \mathbb{T}$. The following four conditions for the angle $\alpha$ are equivalent:

1. $\alpha=\inf G_{s}$.

2. $\alpha \in \mathcal{S}$ such that $G_{s}=G_{\alpha}$.

3. $\alpha=\inf \mathcal{S} \cap[s, 1]$.

4. $\left.I_{0}=\right] 0, \alpha[$.

Proof. $1 \Rightarrow 2$ is a consequence of Lemmas 2.12 and 2.13. The rest is trivial.

Lemma 2.15. Let $a \in \mathcal{S}$. The connected components of $\mathbb{T}-G_{a}$ can be listed as follows: Set inductively $\left.H_{0}=I_{0}=\right] 0, a\left[\right.$, and $H_{n}=\tau^{-1}\left(H_{n-1}\right) \cap$ $[a, 1]$. Then $H_{n}$ is an open set with finitely many connected components $I_{j}$, and $\tau^{n}: I_{j} \rightarrow I_{0}$ is a homeomorphism. For $n \neq m, H_{n} \cap H_{m}=\emptyset$. As a consequence,

$$
\mathbb{T}-G_{a}=\bigsqcup_{n \in \mathbb{N}} H_{n}
$$

Proof. If $n<m$, then $\tau^{n}\left(H_{n}\right)=H_{0}$ and $\tau^{n}\left(H_{m}\right) \subset[a, 1]$. So $H_{n} \cap H_{m}$ $=\emptyset$. The rest of the proof is trivial.

Proposition 2.16. The set $\mathcal{S}$ is closed. The connected components of $\mathbb{T}-\mathcal{S}$ can be listed as (in binary expansions)

$$
\left.\begin{array}{rl}
\mathbb{T}-\mathcal{S} & \left.=\bigsqcup_{k=1}^{\infty} \bigsqcup_{0 . \varepsilon_{1} \ldots \varepsilon_{k} \in \mathcal{S}, \varepsilon_{k}=1}\right] 0 . \varepsilon_{1} \ldots \varepsilon_{k}, 0 . \overline{\varepsilon_{1} \ldots \varepsilon_{k}}[ \\
& \left.=\bigsqcup_{k=1}^{\infty} \bigsqcup_{0 . \varepsilon_{1} \ldots \varepsilon_{k}}\right] 0 . \mathcal{S}
\end{array}\right]
$$

In other words, any connected component of $\mathbb{T}-\mathcal{S}$ must be of the form ] $0 . \varepsilon_{1} \ldots \varepsilon_{k}, 0 . \overline{\varepsilon_{1} \ldots \varepsilon_{k}}\left[, \varepsilon_{k}=1\right.$, and any such interval with at least one of its extremities in $\mathcal{S}$ is a connected component of $\mathbb{T}-\mathcal{S}$. The set $\mathcal{S}$ is perfect, totally disconnected, and of Lebesgue measure zero.

Proof. By definition $\mathcal{S}$ is the set of points $t$ such that $t$ is the smallest in its orbit. Clearly $1,1 / 2 \in \mathcal{S}$, and $] 1 / 2,1[$ is a connected component of $\mathbb{T}-\mathcal{S}$. It is also clear that $\mathbb{T}-\mathcal{S}$ is an open set. So $\mathcal{S}$ is closed.

Let $I=] t, t^{\prime}$ [ be a connected component of $\mathbb{T}-\mathcal{S}, 0<t<t^{\prime}<1$. Let $k$ be the minimal positive integer such that there is a positive integer $p$ with $t \leq p / 2^{k} \leq t^{\prime}$ (such a $p$ is unique). Note that $\tau$ is strictly expanding on any interval contained in $\mathbb{T}-\{1,1 / 2\}$. In particular, $\tau^{i}:\left[t, p / 2^{k}\right] \rightarrow$ $\left[\tau^{i}(t), \tau^{i}\left(p / 2^{k}\right)\right]$ is an expanding homeomorphism, $i=1, \ldots, k-1$. Since 
$\tau^{i}(t) \geq t$ for $i=1, \ldots, k-1$, we have $\tau^{i}\left(p / 2^{k}\right) \geq p / 2^{k}$ for $i=1, \ldots, k-1$. Note that $\tau^{k}\left(p / 2^{k}\right)=1>p / 2^{k}$. So $p / 2^{k} \in \mathcal{S}$. Hence $t=p / 2^{k}$ or $t^{\prime}=p / 2^{k}$.

We now show that $t^{\prime}=p / 2^{k}$ is impossible. If $t^{\prime}=p / 2^{k}$, then $\tau^{k-1} I=$ ]$\tau^{k-1} t, 1 / 2$ [ contains a point $s_{1}$ of the form $\left(2^{n}-1\right) / 2^{n+1}$, which is in $\mathcal{S}$, and then $I$ contains a point $s_{2}$ such that $\tau^{k-1}\left(s_{2}\right)=s_{1}$. It is not difficult to see that $s_{2} \in \mathcal{S}$, we only need to show $\tau^{i}\left(s_{2}\right) \geq s_{2}$ for $i=1, \ldots, k-1$, and this is guaranteed by the same argument as above: for $i=1, \ldots, k-1$, the mapping $\tau^{i}:\left[t, s_{2}\right] \rightarrow\left[\tau^{i}(t), \tau^{i}\left(s_{2}\right)\right]$ is an expanding homeomorphism, and $\tau^{i}(t) \geq t$ since $t \in \mathcal{S}$. But $s_{2} \in \mathcal{S}$ contradicts the choice of $I$.

We conclude that $t=p / 2^{k}$. Now we want to show that for any $p / 2^{k} \in \mathcal{S}$, irreducible, we have $p /\left(2^{k}-1\right) \in \mathcal{S}$ and $] p / 2^{k}, p /\left(2^{k}-1\right)[\subset \mathbb{T}-\mathcal{S}$. (Applying this to $t$, we can conclude that $t^{\prime}=p /\left(2^{k}-1\right)$. As a consequence, $\mathcal{S}$ is perfect, since any isolated point of $\mathcal{S}$ would be on the common boundary of two connected components of $\mathbb{T}-\mathcal{S}$.) First, for $i=1, \ldots, k-1$, the mapping $\tau^{i}:\left[p / 2^{k}, p /\left(2^{k}-1\right)\right] \rightarrow\left[\tau^{i}\left(p / 2^{k}\right), \tau^{i}\left(p /\left(2^{k}-1\right)\right)\right]$ is an expanding homeomorphism, and $\tau^{i}\left(p / 2^{k}\right) \geq p / 2^{k}$; hence $\tau^{i}\left(p /\left(2^{k}-1\right)\right) \geq p /\left(2^{k}-1\right)$. Since $\tau^{k}\left(p /\left(2^{k}-1\right)\right)=p /\left(2^{k}-1\right)$, for any positive integer $n$ we have

$$
\tau^{n}\left(p /\left(2^{k}-1\right)\right)=\tau^{l}\left(p /\left(2^{k}-1\right)\right) \quad \text { for some } 0 \leq l \leq k-1 .
$$

So $p /\left(2^{k}-1\right) \in \mathcal{S}$. Now since $\tau^{k}:\left[p / 2^{k}, p /\left(2^{k}-1\right)\right] \rightarrow\left[0, p /\left(2^{k}-1\right)\right]$ is strictly expanding, for any $\left.t^{\prime \prime} \in\right] p / 2^{k}, p /\left(2^{k}-1\right)\left[\right.$ we have $0<\tau^{k}\left(t^{\prime \prime}\right)<t^{\prime \prime}$. So $t^{\prime \prime} \notin \mathcal{S}$ and then $] p / 2^{k}, p /\left(2^{k}-1\right)[$ is a connected component of $\mathbb{T}-\mathcal{S}$.

The same argument will show that if $p /\left(2^{k}-1\right) \in \mathcal{S}$ for some $p, k$, then ]$p / 2^{k}, p /\left(2^{k}-1\right)[$ is a connected component of $\mathbb{T}-\mathcal{S}$.

The Lebesgue measure of $\mathcal{S}$ will be calculated in Lemma 2.19.

Lemma 2.17. Let $a \in \mathcal{S}$. Assume that a is not a dyadic number, or equivalently a is not the left end point of a connected component of $\mathbb{T}-\mathcal{S}$. Then $G_{a}$ is a perfect set. Let $] \beta, \alpha[$ be a connected component of $] 0,1[-\mathcal{S}$. Assume $\beta=0 . \varepsilon_{1} \varepsilon_{2} \ldots \varepsilon_{k}, \varepsilon_{k}=1$. Then $\beta$ is an isolated point of $G_{\beta}$. Moreover, $G_{\beta}-G_{\alpha}=\bigsqcup_{I} E_{I}$, where the union is taken over all the connected components $I$ of $\mathbb{T}-G_{\alpha}$, and, for $\left.I_{0}=\right] 0, \alpha[$,

$$
E=E_{I_{0}}=\left\{0 . \varepsilon_{1} \ldots \varepsilon_{k}, 0 . \varepsilon_{1} \ldots \varepsilon_{k} \varepsilon_{1} \ldots \varepsilon_{k}, 0 . \varepsilon_{1} \ldots \varepsilon_{k} \varepsilon_{1} \ldots \varepsilon_{k} \varepsilon_{1} \ldots \varepsilon_{k}, \ldots\right\} .
$$

For a general $I$, let $n$ be the integer associated with $I$ defined in Lemma 2.15. Then $E_{I}=\tau^{-n}(E) \cap I$.

The proof is left to the reader.

We give another description of the number $\alpha$ in Corollary 2.14.

Lemma 2.18. Let $s=0 . \varepsilon_{1} \varepsilon_{2} \ldots \in \mathbb{T}$ (in binary expansion). Define a number $t$ as follows: If $s \in \mathcal{S}$, set $t=s$. Otherwise, there is a minimal integer $k$ such that $\left.\tau^{k}(s) \in\right] 0, s\left[\right.$. Set $t=0 . \overline{\varepsilon_{1} \ldots \varepsilon_{k}}$. Then $t=\alpha$. 
Proof. Assume $s \notin \mathcal{S}$. We have $\alpha=\inf \mathcal{S} \cap[s, 1[$, and $\alpha>s$. So $s \in] \beta, \alpha[$ with $] \beta, \alpha\left[\right.$ a connected component of $\mathbb{T}-\mathcal{S}$. If $\alpha=0 . \overline{\sigma_{1} \ldots \sigma_{l}}$, then $\beta=0 . \sigma_{1} \ldots \sigma_{l}$ by Lemma 2.16. It is then easy to check that $s=$ $0 . \sigma_{1} \ldots \sigma_{l} * * \ldots$, and $l$ is the minimal integer such that $\tau^{l}(s)<s$.

Lemma 2.19. For each $a \in \mathbb{T}$, the Lebesgue measure of $G_{a}$ is zero. The set $\mathcal{S}$ also has Lebesgue measure zero.

Pro of. Note that $\tau$ is ergodic with respect to the Lebesgue measure $\mu$. Let $F \subset \mathbb{T}$ with $\mu(F) \neq 0$. Set $G=\left\{t \in \mathbb{T} \mid \tau^{n}(t) \notin F, n=0,1, \ldots\right\}$. Then $G \subset \tau^{-1}(G)$ and $\bigcup_{n \in \mathbb{N}} \tau^{-n}(G)$ is $\tau$-invariant. Hence

$$
\mu\left(\bigcup_{n \in \mathbb{N}} \tau^{-n}(G)\right)=\mu(G) \leq \mu(\mathbb{T}-I)<1 .
$$

By ergodicity, $\mu(G)=0$.

We conclude that $\mu\left(G_{a}\right)=0$. The fact $\mu(\mathcal{S})=0$ follows then from the formula

$$
\mathcal{S}=\bigcup_{n \in \mathbb{N}} \mathcal{S} \cap\left[1 / 2^{n}, 1 / 2\right] \subset \bigcup_{n \in \mathbb{N}} G_{1 / 2^{n}} .
$$

2.3. Head's angle and location of the free critical point. In this subsection we introduce Head's angle for a Newton map $N$ with the Julia set $J_{N}$ locally connected and $x_{0} \notin A_{1} \cup A_{2} \cup A_{3}$. With this angle we will be able to give a combinatorial model of $\partial A_{1} \cap \partial A_{2}$, to point out the location of $x_{0}$ with respect to $A_{1}, A_{2}, A_{3}$ and to describe the dynamics of the connected components of $\overline{\mathbb{C}}-\overline{A_{1} \cup A_{2} \cup A_{3}}$.

Assume in this subsection that $J_{N}$ is locally connected and $x_{0} \notin A_{1} \cup$ $A_{2} \cup A_{3}$.

Definition. The Head's angle of $N$ is defined by

$$
\alpha=\alpha(N)=\inf \left\{t \in \mathbb{T} \mid \iota_{1}(t)=\iota_{2}(-t)\right\} .
$$

By Corollary 2.8, $\alpha>0$. Since $T_{N}$ is closed, $\alpha \in T_{N}$. Thus $\bar{a}_{1}(0) \cup$ $\bar{a}_{2}(0) \cup \bar{a}_{1}(\alpha) \cup \bar{a}_{2}(-\alpha)$ forms a Jordan curve.

The sets $\mathcal{S}$ and $G_{\alpha}$ were defined and studied in Subsection 2.2.

Proposition 2.20. We have $\alpha \in \mathcal{S}$ and $T_{N}=G_{\alpha}$. In other words, $\iota_{1}$ gives rise to a homeomorphism from $G_{\alpha}$ to $\partial A_{1} \cap \partial A_{2}$ and conjugates $\tau$ to $N$.

Pr o of. We check that $T_{N}$ satisfies the assumptions of Lemma 2.13. Let $I=] t, t^{\prime}\left[\right.$ be a connected component of $\mathbb{T}-T_{N}$, and $\left.I \neq\right] 0, \alpha[$. We have $1,1 / 2 \notin I$, and $\tau(t), \tau\left(t^{\prime}\right) \in T_{N}$. We want to show that $] \tau(t), \tau\left(t^{\prime}\right)\left[\cap T_{N}=\emptyset\right.$. Let $t<s<t^{\prime}$. If $\tau(s) \in T_{N}$, then $N\left(\iota_{1}(s)\right)=\iota_{1}(\tau(s)) \in \partial A_{2}$. So $\iota_{1}(s) \in$ $\partial A_{1} \cap \partial W_{2}$. 
By looking at the position of $\iota_{1}(\alpha / 2)$ (which is also a point of $\partial W_{2}$ ) and $\iota_{1}(s)$, we can see easily that $W_{2}$ and $\iota_{1}(s)$ are in different connected components of $\overline{\mathbb{C}}-\bar{a}_{1}(0) \cup \bar{a}_{2}(0) \cup \bar{a}_{1}(\alpha) \cup \bar{a}_{2}(-\alpha)$. So $\iota_{1}(s) \in \partial A_{2}$ and $s \in T_{N}$. But this contradicts the choice of $I$.

Remark. The converse question is: given any angle $\alpha \in \mathcal{S}$, is there a Newton map with Head's angle $\alpha$ ? We will see in the following sections that the answer is affirmative for $\alpha$ rational. (We conjecture that the answer is also affirmative for $\alpha$ irrational. But to construct such Newton maps we have to deal with postcritically infinite rational maps.) Moreover, for any periodic angle $\alpha \in \mathcal{S}$, there are many Newton maps with Head's angle $\alpha$. Hence one important observation of the above theorem is that the set $T_{N}$ (or $\partial A_{1} \cap \partial A_{2}$ ) does not depend on the special choice of $N$, but only on the angle $\alpha$.

From Lemma 2.17 we get immediately the following two corollaries:

Corollary 2.21 ([He], Lemma 3.6.2). The point $\iota_{1}(\alpha)$ is isolated in $\partial A_{1} \cap \partial A_{2}$ iff $\alpha$ is dyadic.

Corollary 2.22. $\partial A_{1} \cap \partial W_{2}$ is homeomorphic to

$$
\begin{aligned}
\left\{t \in \mathbb{T} \mid \iota_{1}(t)=\sigma_{2}(-2 t)\right\} & =\tau^{-1}\left(T_{N}\right)-T_{N} \\
& =\tau^{-1}\left(T_{N}\right) \cap\left[\alpha / 2, \alpha\left[=\left\{t / 2 \mid t \in T_{N} \cap[\alpha, 2 \alpha[\},\right.\right.\right.
\end{aligned}
$$

and similarly for $\partial A_{2} \cap \partial W_{1}$. The set $\partial W_{1} \cap \partial W_{2}$ is homeomorphic to $T_{N} \cap[2 \alpha, 1]$.

Proposition 2.23 ([He], Lemma 3.6.2). The following conditions are equivalent:

1. $x_{0}=\iota_{1}(\alpha)$.

2. $\alpha$ is not periodic.

Proof. If a critical point belongs to the Julia set then it cannot be periodic, otherwise we would have a superattracting cycle, which is disjoint from the Julia set.

If $\alpha$ is not periodic, define $\beta=\max \left\{t \mid \iota_{1}(t)=\sigma_{2}(-2 t)\right\}$. Then $\beta=\alpha$, for otherwise $] \tau(\beta), \tau(\alpha)$ [ is a connected component of $\mathbb{T}-T_{N}=\mathbb{T}-G_{\alpha}$. But the right end point of each connected component of $\mathbb{T}-G_{\alpha}$ is a preimage by $\tau$ of $\alpha$. Hence $\iota_{1}(\alpha) \in \bar{a}_{2}(-\alpha) \cap \bar{w}_{2}(-2 \alpha)$. That is, $N$ is not injective in a neighborhood of $\iota_{1}(\alpha)$. Since $x_{0}$ is the only free critical point of $N$, we must have $x_{0}=\iota_{1}(\alpha)$.

Assume now $\alpha$ is periodic. Define

$$
\beta=\inf \left\{t \in \mathbb{T} \mid \iota_{1}(s) \notin \bar{W}_{2} \text { for all } s \in[t, 1]\right\} .
$$


Lemma 2.24 ([He], Lemma 3.6.3). Let $\alpha=p /\left(2^{k}-1\right)$. Then $] \tau(\beta), \tau(\alpha)[$ is a connected component of $\mathbb{T}-T_{N}$, and $\beta=p / 2^{k}$. In other words, $] \beta, \alpha[$ is the connected component of $\mathbb{T}-\mathcal{S}$ with $\alpha$ as the right end point.

Pr o of. We have $\beta<\alpha$ and $\iota_{1}(\beta) \in \partial W_{2}$. So $] \tau(\beta), \tau(\alpha)$ [ is a connected component of $\mathbb{T}-T_{N}=\mathbb{T}-G_{\alpha}$. Hence $] \tau^{k}(\beta), \tau^{k}(\alpha)[=] 0, \alpha[($ Lemma 2.15). Thus $\beta \in \mathcal{S}$ and $\tau^{k}(t)<t$ for all $\left.t \in\right] \beta, \alpha[$. We conclude that $] \beta, \alpha[\cap \mathcal{S}=\emptyset$. The rest follows from Proposition 2.16.

Denote by $U$ the union of the open bounded components surrounded by the following four arcs:

$$
\begin{array}{ll}
\gamma_{0}=\left\{\iota_{1}(t) \mid \beta \leq t \leq \alpha\right\}, & \eta_{0}=\left\{\iota_{2}(t) \mid 1-\alpha \leq t \leq 1-\beta\right\}, \\
\widetilde{\gamma}_{0}=\left\{\sigma_{1}(t) \mid 2 \beta \leq t \leq 2 \alpha\right\}, & \widetilde{\eta}_{0}=\left\{\sigma_{2}(t) \mid 1-2 \alpha \leq t \leq 1-2 \beta\right\} .
\end{array}
$$

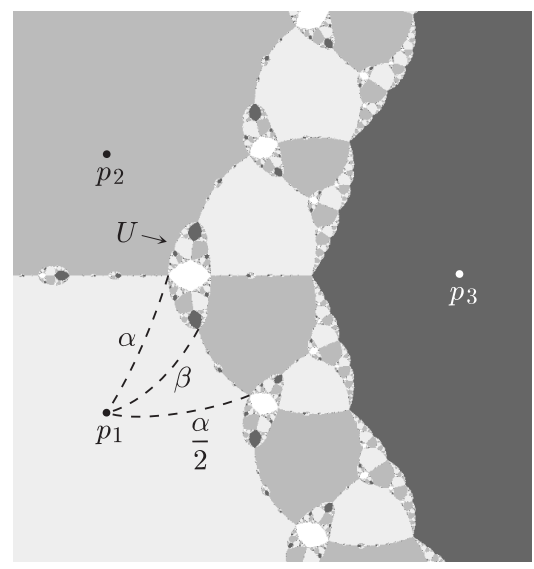

Fig. $5^{\prime} . \alpha$-ray, $\beta$-ray and the region $U$

Proposition 2.25 ([He]). Assume that $\alpha$ is periodic. If $U$ is connected, then $x_{0} \in U$, the set $N(U)$ is a connected component of $\overline{\mathbb{C}}-\overline{A_{1} \cup A_{2}}$, and $N$ induces a degree two covering from $U$ to $N(U)$, ramified at $x_{0}$. The following statements are equivalent:

1. $U$ is not connected.

2. $U$ has two connected components $U_{1}, U_{2}$ and $\bar{U}_{1} \cap \bar{U}_{2}=\left\{x_{0}\right\}$.

3. Either $x_{0}=\iota_{1}(t)$ or $x_{0}=\iota_{2}(-t)$ for some $\beta<t<\alpha$.

In this case $N\left(U_{1}\right)=N\left(U_{2}\right)$ is a connected component of $\overline{\mathbb{C}}-\overline{A_{1} \cup A_{2}}$.

The proof is left to the reader.

Corollary 2.26. Suppose that $J_{N}$ is locally connected and $x_{0}$ is not contained in $A_{1} \cup A_{2} \cup A_{3}$. Let $\alpha$ be the Head's angle of $N$. There is a bijection between connected components of $\mathbb{T}-G_{\alpha}$ and connected components 
in $\overline{\mathbb{C}}-\bar{A}_{1} \cup \bar{A}_{2}$. More precisely, let $\left.I=\right] t, t^{\prime}[$ be a connected component of $\mathbb{T}-G_{\alpha}$. Then $\left\{\iota_{1}(s) \mid s \in\left[t, t^{\prime}\right]\right\} \cup\left\{\iota_{2}(-s) \mid s \in\left[t, t^{\prime}\right]\right\}$ forms a Jordan curve, with one connected component $E_{I}$ disjoint from $\bar{A}_{1} \cup \bar{A}_{2}$. For $\left.I_{0}=\right] 0, \alpha[$, we have $E_{I_{0}} \supset A_{3} \cup W_{1} \cup W_{2}$. For $I \neq I_{0}, N: E_{I} \rightarrow E_{\tau(I)}$ is a homeomorphism.

Denote by $H$ the set $E_{I_{0}}-\bar{A}_{3}$. It is an open connected set. Denote by $H_{1,2}$ the two connected components of $H-\bar{W}_{1} \cup \bar{W}_{2}$ with $\infty$ as a point on the boundary. Recall that $E_{I}$ is defined in the above corollary, and $\infty$ is the unique common point of $\bar{A}_{1}, \bar{A}_{2}, \bar{A}_{3}$.

Corollary 2.27. The mapping $N$ induces a homeomorphism from $H_{1,2}$ to $H$. For any connected component $F$ of $H-\bar{W}_{1} \cup \bar{W}_{2}$ with neither $\infty$ nor $\iota_{1}(\alpha)$ on the boundary, the mapping $N$ induces a homeomorphism from $F$ to some $E_{t, t t^{\prime}}$, with $t$ greater than $\alpha$. The angle $t^{\prime}$ is smaller than $2 \alpha$ iff the boundary of $F$ intersects the closure of $A_{1}$ or $A_{2}$. If $\alpha$ is periodic, then the set $U\left(\right.$ resp. $\left.U_{1}, U_{2}\right)$ as in Proposition 2.25 is a connected component of $H-\bar{W}_{1} \cup \bar{W}_{2}$, and $N(U)$ (resp. $\left.N\left(U_{1}\right), N\left(U_{2}\right)\right)$ is equal to $E_{] 2 \beta, 2 \alpha[}$.

Corollary 2.28 ([He]). Suppose that $\alpha$ is periodic of period $k$. Then there is an open neighborhood $U^{\prime}$ of $\bar{U}$ such that $U^{\prime}$ is relatively compact in $N^{k}\left(U^{\prime}\right)$ and $\left(N^{k}, U^{\prime}, N^{k}\left(U^{\prime}\right)\right)$ is a polynomial-like mapping of degree two (in the sense of Douady-Hubbard, [DH3]), with $\iota_{1}(\alpha)$ as one fixed point of the corresponding Julia set.

P r o of. Note that $\iota_{1}(\alpha)$ is an accumulation point of $\partial A_{1} \cap \partial A_{2}$, and $\iota_{1}(\beta)$ is an accumulation point of $\partial A_{1} \cap \partial W_{2}$ (Corollary 2.21). We can then construct the boundary of $U^{\prime}$ with part of equi-potentials and part of rays with angle close to $\alpha, \beta$. We omit the details.

One can deduce even deeper properties of $H_{1,2}$ by considering the homeomorphisms $N: H_{1,2} \rightarrow H$. We again omit the details.

Here we state our conjecture about islands and Head's angles (we use the notations defined in Section 1):

Conjecture. Given any component $] \beta, \alpha[$ of $\mathbb{T}-\mathcal{S}$, different from ] $1 / 2,1\left[\right.$, there is a unique connected component of $\Omega-\overline{B_{0} \cup G_{0}}$ such that the maps in the closure of this component are precisely the maps with Head's angle $\beta$ or $\alpha$.

In the rest of the paper we will only be interested in postcritically finite Newton maps (i.e. $x_{0}$ has a finite orbit). They have locally connected Julia sets (cf. [TY]), and a well defined rational Head's angle, when $x_{0} \notin$ $A_{1} \cup A_{2} \cup A_{3}$.

3. Cubic branched coverings with three fixed critical points. We start the construction of postcritically finite Newton maps. The technique 
is to construct first postcritically finite branched coverings from $S^{2}$ to itself, and then deduce the existence of rational maps via Thurston equivalence (see definition below). By the end of this paper we will be able to construct all postcritically finite Newton maps.

3.1. Definition and Thurston equivalence. We recall here Thurston's theory of postcritically finite branched coverings. The results are valid for any degree. For details and proofs, see [DH2].

A branched covering $F: S^{2} \rightarrow S^{2}$ of degree $d$ has in general $2 d-2$ critical points and $2 d-2$ critical values. In the following, we always assume that branched coverings are orientation preserving and of degree greater than one.

Definition (postcritical set). Set

$$
\Omega_{F}=\{\text { critical points of } F\} \quad \text { and } \quad P_{F}=\bigcup_{n>0} F^{n}\left(\Omega_{F}\right)
$$

$F$ is called a postcritically finite branched covering if $P_{F}$ is finite.

Definition (Thurston's equivalence). We say that two postcritically finite branched coverings $F$ and $G$ are equivalent, denoted by $F \sim G$, if there exist two orientation preserving homeomorphisms $\theta_{1}, \theta_{2}: S^{2} \rightarrow S^{2}$ such that

$$
\begin{aligned}
& \theta_{i}\left(P_{F}\right)=P_{G}(i=1,2), \\
& \theta_{1}=\theta_{2} \text { on } P_{F}, \theta_{1} \text { and } \theta_{2} \text { are isotopic relative to } P_{F}
\end{aligned}
$$

(we write rel $P_{F}$ ), and the following diagram commutes:

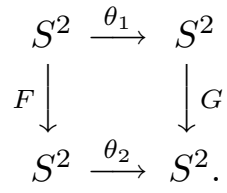

We are interested in whether a branched covering is equivalent to a rational map in the above sense. Let $F$ be a postcritically finite branched covering from $S^{2}$ to itself. All the homotopies and isotopies in the following are rel $P_{F}$.

Definition. A simple closed curve in $S^{2}-P_{F}$ is called peripheral if it bounds a disc containing at most one point of $P_{F}$. A multicurve $\Gamma$ is a collection of disjoint simple closed curves in $S^{2}-P_{F}$ such that none of them is peripheral and no two curves are homotopic to each other in $S^{2}-P_{F}$. A multicurve $\Gamma$ is $F$-invariant if

$$
F^{-1}(\Gamma)=\left\{\text { connected components of } F^{-1}(\gamma) \mid \gamma \in \Gamma\right\}
$$

consists of peripheral curves and curves which are homotopic to curves in $\Gamma$. 
Definition. For a multicurve $\Gamma$, the associated linear transformation $F_{\Gamma}$ is a linear map from $\mathbb{R}^{\Gamma}=\left\{\sum_{\gamma \in \Gamma} c_{\gamma} \gamma \mid c_{\gamma} \in \mathbb{R}\right\}$ to itself defined by

$$
F_{\Gamma}(\gamma)=\sum_{\gamma^{\prime} \subset F^{-1}(\gamma)} \frac{1}{\operatorname{deg}\left(F: \gamma^{\prime} \rightarrow \gamma\right)}\left[\gamma^{\prime}\right]_{\Gamma} \quad \text { for } \gamma \in \Gamma
$$

where the sum is over all components $\gamma^{\prime}$ of $F^{-1}(\gamma)$, and $\left[\gamma^{\prime}\right]_{\Gamma}$ denotes the curve in $\Gamma$ homotopic to $\gamma^{\prime}$ if it exists and $\left[\gamma^{\prime}\right]_{\Gamma}=0$ otherwise. We denote by $\lambda_{\Gamma}$ the leading eigenvalue of $F_{\Gamma}$, i.e. the Perron-Frobenius eigenvalue.

Definition. 1. An $F$-invariant multicurve $\Gamma$ with $\lambda_{\Gamma} \geq 1$ is called a Thurston obstruction for $F$.

2. A Thurston obstruction $\Gamma$ for $F$ is called minimal if every $\gamma \in \Gamma$ is homotopic to a curve of $F^{-1}(\Gamma)$, and any invariant proper submulticurve of $\Gamma$ has leading eigenvalue less than one.

3. A multicurve $\Gamma=\left\{\gamma_{1}, \ldots, \gamma_{n}\right\}$ is called a Levy cycle if each $F^{-1}\left(\gamma_{i+1}\right)$ contains a component $\gamma_{i}^{\prime}$ homotopic in $S^{2}-P_{F}$ to $\gamma_{i}$ and $F: \gamma_{i}^{\prime} \rightarrow \gamma_{i+1}$ is of degree one $(i=0, \ldots, n-1)$, where $\gamma_{0}=\gamma_{n}$.

4. A Levy cycle $\Gamma$ is a degenerate Levy cycle if the connected components of $S^{2}-\bigcup_{i} \gamma_{i}$ are

$$
B_{1}, B_{2}, \ldots, B_{n}, C
$$

with $B_{i}$ discs, $C$ not a disc, and each $F^{-1}\left(B_{i+1}\right)$ has a component $B_{i}^{\prime}$ isotopic to $B_{i}\left(\right.$ rel $\left.P_{F}\right)$, and $F: B_{i}^{\prime} \rightarrow B_{i+1}$ is a homeomorphism $(i=0,1, \ldots, n-1)$, where $B_{0}=B_{n}(C=\emptyset$ if $\Gamma$ consists of only one curve $)$.

Note that every Thurston obstruction can be reduced to a minimal one.

TheOREM 3.1 (Thurston, cf. [DH2]). Suppose that $F: S^{2} \rightarrow S^{2}$ is a postcritically finite branched covering with a hyperbolic orbifold (see [DH2] for definition). Then $F$ is equivalent to a rational map if and only if $F$ has no Thurston obstruction. Having a Levy cycle is a sufficient condition for having a Thurston obstruction.

Applying the theorem to the case that interests us in this work, we get

COROLLARY 3.2. Every cubic postcritically finite branched covering with three fixed critical points has a hyperbolic orbifold. If it is Thurston-equivalent to a rational map $N$, then $N$ must be a Newton map, i.e. a cubic rational map with three fixed critical points.

3.2. From a Thurston obstruction to a one-curve Levy cycle. In this section we will show that if a cubic postcritically finite branched covering fixing at least 3 critical points is not equivalent to a rational map, it has either a degenerate Levy cycle or an extremely simple Levy cycle, namely one with only one curve. 
Let $F$ be a cubic postcritically finite branched covering from $S^{2}$ to itself. All the homotopies and isotopies in the following are rel $P_{F}$.

A multicurve $\Gamma_{1}$ may have the following properties:

(I) Each $\gamma \in \Gamma_{1}$ is homotopic to a curve in $F^{-1}\left(\Gamma_{1}\right)$.

(II) For each $\gamma \in \Gamma_{1}$, at least one component of $F^{-1}(\gamma)$ is homotopic to a curve in $\Gamma_{1}$.

(III) For some disc-component $D$ of $S^{2}-\Gamma_{1}$, the set $F^{-1}(D)$ has at least one non-disc component.

(IV) For a connected component $B$ of $S^{2}-\Gamma_{1}$, every component of $F^{-1}(B)$ is isotopically contained in a component of $S^{2}-\Gamma_{1}$.

Note that (IV) is a consequence of (I). It is going to be used many times in what follows.

THEOREM 3.3. Assume that $F$ is a cubic postcritically finite branched covering with three fixed distinct critical points $p_{1}, p_{2}, p_{3}$. Denote by $x_{0}$ the other critical point and by $x_{1}=F\left(x_{0}\right)$ the critical value. Suppose that $F$ has a minimal Thurston obstruction $\Gamma$. Then at least one of the following happens:

1. $\Gamma$ contains a degenerate Levy cycle.

2. $\Gamma$ can be decomposed into the disjoint union of $\{\gamma\}$ and $\Gamma_{2}$ with $\{\gamma\}$ a Levy cycle, and $\Gamma_{2} F$-invariant (hence $\lambda\left(\Gamma_{2}\right)<1$ ). Moreover, each component of $S^{2}-\gamma$ contains exactly two of the four critical values $p_{1}, p_{2}, p_{3}, x_{1}$, $F^{-1}(\gamma)$ consists of three nested curves $\gamma^{\prime}, \gamma^{\prime \prime}, \gamma^{\prime \prime \prime}$ with $\gamma^{\prime \prime}$ homotopic to $\gamma$ (with the same orientation) and $\gamma^{\prime}, \gamma^{\prime \prime \prime}$ either peripheral or homotopic to curves in $\Gamma_{2}$ (see Figure 6).
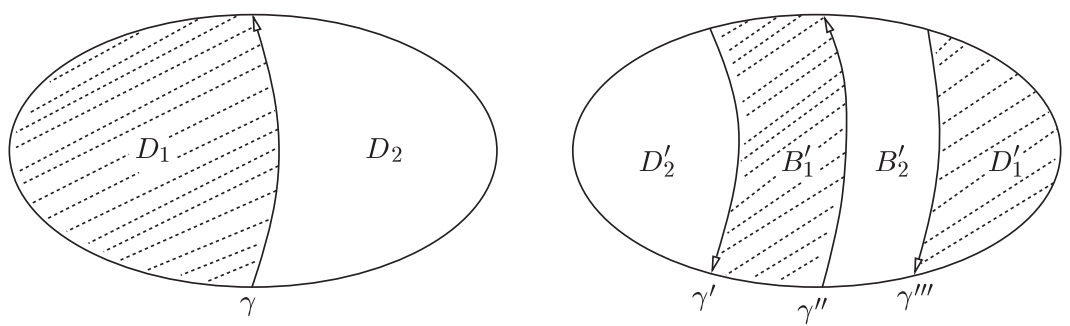

Fig. 6. $\gamma, F^{-1}(\gamma)=\gamma^{\prime} \cup \gamma^{\prime \prime} \cup \gamma^{\prime \prime \prime}, D_{i}$ and $F^{-1}\left(D_{i}\right)=D_{i}^{\prime} \cup B_{i}^{\prime}, i=1,2$

Before proving the theorem, we rewrite Theorem III.3.4 of [ST] in the following more convenient form:

Proposition 3.4 (minimal-maximal principle; [ST], see also [Ta]). Let $F$ be a postcritically finite branched covering, and $\Gamma$ a minimal Thurston obstruction for $F$. Then $\Gamma$ can be decomposed in a unique way into $\Gamma_{1} \cup \Gamma_{2}$ 
with $\Gamma_{2}$ the maximal invariant proper submulticurve of $\Gamma$ ( $\Gamma_{2}$ may be empty). Either $\Gamma_{1}$ is a degenerate Levy cycle or $\Gamma_{1}$ has the properties (I)-(IV).

Proof (of Theorem 3.3). Assume that $\Gamma$ does not contain any degenerate Levy cycle. Then $\Gamma$ is decomposed into $\Gamma_{1} \cup \Gamma_{2}$, with $\Gamma_{2}$ the maximal invariant proper submulticurve and $\Gamma_{1}$ satisfying (I)-(IV).

Note first that if a subset $B \subset S^{2}$ satisfies $B \cap\left\{p_{1}, p_{2}, p_{3}\right\}=\emptyset$, then $F^{-1}(B) \cap\left\{p_{1}, p_{2}, p_{3}\right\}=\emptyset$. Moreover, if $B$ is disc-like, then $B$ contains at most one critical value of $F$ and hence $F^{-1}(B)$ are discs.

Set

$$
\begin{aligned}
& \Gamma^{\prime}=\left\{\gamma \in \Gamma \mid \gamma=\partial D, D \text { a disc-component of } S^{2}-\Gamma,\right. \\
& \left.\qquad D \cap\left\{p_{1}, p_{2}, p_{3}\right\}=\emptyset\right\} .
\end{aligned}
$$

Then $\Gamma^{\prime}$ is $F$-invariant. We have $\Gamma^{\prime} \neq \Gamma$, for otherwise one could decompose $\Gamma^{\prime}$ so that a submulticurve of $\Gamma^{\prime}$ would satisfy (III) (by Proposition 3.4). This is impossible from the definition of $\Gamma^{\prime}$. Hence $\Gamma^{\prime} \subset \Gamma_{2}$. Set

$$
S^{2}-\Gamma_{1}=D_{1} \cup \ldots \cup D_{k} \cup A_{1} \cup \ldots \cup A_{l}
$$

where the $D_{i}$ denote the disc-components of $S^{2}-\Gamma_{1}$ and $A_{i}$ the non-disc components. We have $k \geq 2$. We claim

Lemma 3.5. Each $D_{i}$ contains exactly two critical values of $F$.

Proof. First for each $i, D_{i} \cap\left\{p_{1}, p_{2}, p_{3}\right\} \neq \emptyset$. Otherwise the union of $\Gamma_{2}$ with

$$
\Gamma^{\prime \prime}=\left\{\partial D_{i} \mid D_{i} \cap\left\{p_{1}, p_{2}, p_{3}\right\}=\emptyset\right\}
$$

is $F$-invariant. Hence $\Gamma^{\prime \prime}=\Gamma_{1}$ (since $\Gamma_{2}$ is maximal). But $\Gamma^{\prime \prime}$ does not satisfy (III), a contradiction.

Next, if there is $D=D_{i}$ containing only one of $p_{1}, p_{2}, p_{3}$, say $p_{1}$, and if $x_{1} \notin D$, then $F^{-1}(D)=D^{\prime} \cup D^{\prime \prime}$ with $D^{\prime}, D^{\prime \prime}$ disc-like, $\operatorname{deg}\left(F: D^{\prime} \rightarrow D\right)$ $=1, D^{\prime} \cap\left\{p_{1}, p_{2}, p_{3}\right\}=\emptyset$ and $\operatorname{deg}\left(F: D^{\prime \prime} \rightarrow D\right)=2, p_{1} \in D^{\prime \prime}$. By property (IV), $D^{\prime}$ is isotopically contained in one component $B$ of $S^{2}-\Gamma_{1}$. But $D^{\prime}$ cannot be isotopic to $B$ because either $B$ is one of $D_{j}$ in which case $B \cap\left\{p_{1}, p_{2}, p_{3}\right\} \neq \emptyset$, or $B$ is not a disc. So $\partial D^{\prime}$ is not homotopic to any curve in $\Gamma_{1}$. By property (II), the curve $\partial D^{\prime \prime}$ must be homotopic to some curve in $\Gamma_{1}$. By (IV), $D^{\prime \prime}$ must be isotopically contained in $D$, which is a disc-component of $S^{2}-\Gamma_{1}$. We conclude that $\partial D^{\prime \prime}$ is homotopic to $\partial D$. As a consequence, $\{\partial D\} \cup \Gamma_{2}$ is $F$-invariant with leading eigenvalue equal to $\max \left\{1 / 2, \lambda_{\Gamma_{2}}\right\}$, which is smaller than 1 . This contradicts the maximality of $\Gamma_{2}$. $\mathbf{m}_{\text {Lemma } 3.5}$

Let us return to the proof of Theorem 3.3. We have $k=2$ and we may assume $x_{1}, p_{3} \in D_{1}, p_{1}, p_{2} \in D_{2}$. Set $\gamma_{1}=\partial D_{1}$ and $\gamma_{2}=\partial D_{2}$. For $i=1$ or $i=2$, there are two possibilities for $F^{-1}\left(D_{i}\right)$ : either 
(a) $F^{-1}\left(D_{i}\right)$ consists of only one disc $D_{i}^{\prime}$ with $\operatorname{deg}\left(F: D_{i}^{\prime} \rightarrow D_{i}\right)=3$, or

(b) $F^{-1}\left(D_{i}\right)=D_{i}^{\prime} \cup B_{i}^{\prime}$, with $D_{i}^{\prime}$ a disc, $B_{i}^{\prime}$ an annulus, $\operatorname{deg}\left(F: D_{i}^{\prime} \rightarrow D_{i}\right)$ $=1$ and $\operatorname{deg}\left(F: B_{i}^{\prime} \rightarrow D_{i}\right)=2$. Moreover, $B_{i}^{\prime} \cap\left\{p_{1}, p_{2}, p_{3}\right\}=D_{i} \cap$ $\left\{p_{1}, p_{2}, p_{3}\right\} \neq \emptyset$.

If (a) happens for some $i$ then $D_{i}^{\prime}$ is isotopic to $D_{i}$ by the condition (IV) and $\partial D_{i}^{\prime}$, being the only preimage of $\partial D_{i}$, is homotopic to itself by (II). Thus $\left\{\partial D_{i}\right\} \cup \Gamma_{2}$ is $F$-invariant with leading eigenvalue equal to $\max \left\{1 / 3, \lambda_{\Gamma_{2}}\right\}$ $<1$. This contradicts again the maximality of $\Gamma_{2}$.

Hence (b) is the only possibility for both $D_{1}$ and $D_{2}$.

Suppose now $\gamma_{1} \neq \gamma_{2}$. Set

$$
\begin{gathered}
A=S^{2}-\bar{D}_{1} \cup \bar{D}_{2}, \quad F^{-1}(A)=A_{1} \cup A_{2} \cup A_{3}, \\
F^{-1}\left(\gamma_{1}\right)=\gamma_{11} \cup \gamma_{12} \cup \gamma_{13}, \quad F^{-1}\left(\gamma_{2}\right)=\gamma_{21} \cup \gamma_{22} \cup \gamma_{23} .
\end{gathered}
$$

Then the situation is shown in Figure 7.
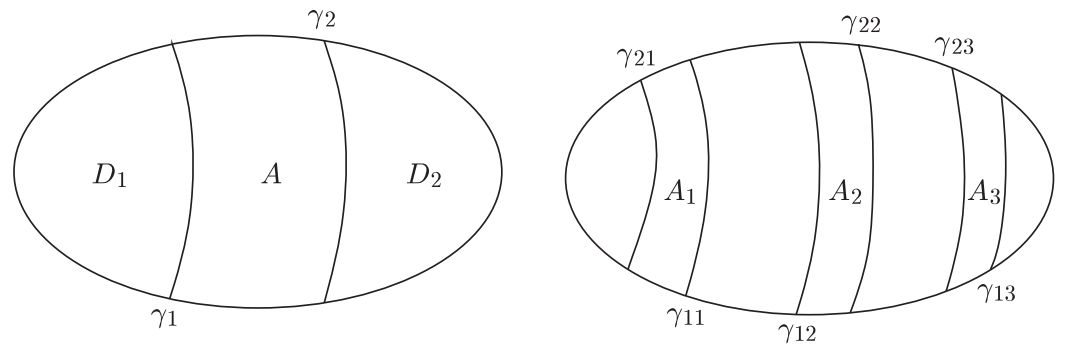

Fig. 7. $F^{-1}(A)$ and $F^{-1}\left(D_{i}\right)$

Since $B_{i}^{\prime}$ must be isotopically contained in $D_{i}, i=1,2$ (condition (IV)), we find that $\gamma_{21}, \gamma_{11}, \gamma_{23}, \gamma_{13}$ are either peripheral or homotopic to curves in $\Gamma_{2}$. By (II) we must have $\gamma_{12}$ homotopic to $\gamma_{1}$ and $\gamma_{22}$ homotopic to $\gamma_{2}$. Hence $\left\{\gamma_{1}\right\} \cup \Gamma_{2}$ is $F$-invariant with leading eigenvalue 1 . This contradicts the minimality of $\Gamma$.

Finally, $\gamma_{1}$ equals $\gamma_{2}$ and $\Gamma_{1}$ consists of only one curve, namely $\gamma_{1}$. Moreover, the connected components of $F^{-1}\left(\gamma_{1}\right)$ are disposed as in Figure 6.

R e m a r k. We will see later that degenerate Levy cycles do not occur as Thurston obstructions for branched coverings constructed from matings and captures (see next two sections). The next subsection improves the above theorem under a stronger condition.

3.3. No Thurston obstruction when the basins are attached. Often we want to construct a postcritically finite Newton map with a specific dynamics; for example, we may want $x_{6}=\lambda_{3}$ or so. Since it is much easier to construct a postcritically finite branched covering than a rational map, we start with that branched covering and check if it is equivalent to a rational map. 
The next theorem is developed for this purpose. Both the statement and the proof are similar to a theorem of J. Head ([He], Theorem 4.4.1). The author would like to thank M. Shishikura for having simplified the original proof.

TheOREm 3.6. Let $F: S^{2} \rightarrow S^{2}$ be a cubic postcritically finite branched covering with three distinct fixed critical points $p_{1}, p_{2}$ and $p_{3}$. Assume that there are disjoint open connected subsets $A_{1}, A_{2}$ and $A_{3}$ in $S^{2}$, together with continuous maps $\varphi_{i}$ from the closed unit disc $\bar{D}$ to the closure of $A_{i}$, $i=1,2,3$, such that $\varphi_{i}: D \rightarrow A_{i}$ is a homeomorphism, $\varphi_{1}(1)=\varphi_{2}(1)=$ $\varphi_{3}(1)=\infty$, and for $i=1,2,3$, the following diagram commutes:

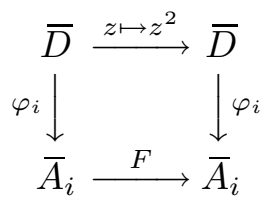

Then either $F$ has a degenerate Levy cycle, or $F$ is equivalent to a Newton map.

Proof. Under the assumption of the theorem we can define $W_{i}$ as the connected component of $F^{-1}\left(A_{i}\right)$ disjoint from $A_{i}$, for $i=1,2,3$. Similarly, we can define $\xi_{1}, \xi_{2}, \infty$ as the first preimages by $F$ of $\infty$. For $i=1,2,3$, the mapping $F: \bar{W}_{i} \rightarrow \bar{A}_{i}$ is a homeomorphism. Set $\psi_{i}=F^{-1} \circ \varphi_{i}$. The mappings $\varphi_{i}$ and $\psi_{i}$ enable us to define $t$-rays in $\bar{A}_{i}$ and $\bar{W}_{i}$, for any angle $t \in \mathbb{T}$. By the same topological argument as we used in Lemma 2.5, one can get similarly

$$
\xi_{1} \in \partial A_{1} \cap \partial A_{2} \cap \partial W_{3}, \quad \xi_{2} \in \partial W_{1} \cap \partial W_{2} \cap \partial A_{3} .
$$

We can renumber $p_{1}, p_{2}$ if necessary so that the 0-rays of $\bar{A}_{1}, \bar{A}_{2}, \bar{A}_{3}$ are connected at $\infty$ in counterclockwise order.

Denote by $x_{0}$ the unique critical point of $F$ distinct from $p_{1}, p_{2}, p_{3}$. Set $x_{i}=F^{i}\left(x_{0}\right)$. For any $t \in \mathbb{T}$, denote by $a_{i}(t)$ the closure of the $t$-ray of $A_{i}$, and by $w_{i}(t)$ the closure of the $t$-ray of $W_{i}(i=1,2,3)$. We have $F^{-1}\left(a_{i}(t)\right)=a_{i}(t / 2) \cup a_{i}(t / 2+1 / 2) \cup w_{i}(t)$.

We claim that the angle $\alpha$ is positive, where

$$
\alpha=\inf \left\{t \in \mathbb{T} \mid a_{1}(t) \cap \bar{A}_{2} \neq \emptyset\right\} .
$$

For otherwise there is a sequence of positive angles $t_{n}$ tending to 0 such that $a_{1}\left(t_{n}\right)$ intersects $a_{2}\left(-t_{n}\right)$. But the union $a_{1}\left(t_{n}\right) \cup a_{2}\left(t_{n}\right)$ tends to the arc $a_{1}(0) \cup a_{2}(0)$. There will be no place to put $A_{3}$ in.

Thus there is a minimal integer $l$ such that

$$
a_{1}\left(1 / 2^{l}\right) \cap w_{2}\left(-1 / 2^{l-1}\right) \neq \emptyset
$$

(compare with Lemma 2.7). 
Assume now that $F$ is not equivalent to a rational map, and $F$ has no degenerate Levy cycle. By Theorem 3.3 there is a one-curve Levy cycle $\{\gamma\}$ for $F$ such that each component of $S^{2}-\gamma$ contains two critical values of $F$. And $F^{-1}(\gamma)=\gamma^{\prime} \cup \gamma^{\prime \prime} \cup \gamma^{\prime \prime \prime}$ (Figure 6) with $\gamma^{\prime \prime}$ the unique component in $F^{-1}(\gamma)$ homotopic to $\gamma$. Set $\delta=\gamma^{\prime \prime}$. Denote by $[\gamma]$ the set of closed curves in $S^{2}$ homotopic to $\gamma$ rel $P_{F}$.

First we want to show that $\gamma$ can be chosen such that

$$
\gamma \cap\left(a_{1}(1 / 2) \cup a_{2}(1 / 2) \cup w_{1}(0) \cup w_{2}(0)\right)=\emptyset .
$$

Choose $\gamma \in[\gamma]$ such that $\# \gamma \cap\left(a_{1}(0) \cup a_{2}(0)\right)$ is minimal. Then

$$
\# \delta \cap\left(a_{1}(0) \cup a_{2}(0)\right) \leq \# F^{-1}(\gamma) \cap\left(a_{1}(0) \cup a_{2}(0)\right)=\# \gamma \cap\left(a_{1}(0) \cup a_{2}(0)\right) \text {. }
$$

(It is easy to see that for any two sets $A, B$ such that $\left.F\right|_{B}$ is of degree one we have $F\left(F^{-1}(A) \cap B\right)=A \cap F(B)$ and $\# F^{-1}(A) \cap B=\# A \cap F(B)$.) But since $\delta \in[\gamma]$, we have $\# \delta \cap\left(a_{1}(0) \cup a_{2}(0)\right) \geq \# \gamma \cap\left(a_{1}(0) \cup a_{2}(0)\right)$. Hence

$$
\# \delta \cap\left(a_{1}(0) \cup a_{2}(0)\right)=\# F^{-1}(\gamma) \cap\left(a_{1}(0) \cup a_{2}(0)\right)=\# \gamma \cap\left(a_{1}(0) \cup a_{2}(0)\right) \text {. }
$$

Since

$$
\begin{aligned}
& \# \gamma \cap\left(a_{1}(0) \cup a_{2}(0)\right)=\# \delta \cap\left(a_{1}(0) \cup a_{2}(0)\right) \\
& \quad \leq \# \delta \cap\left(a_{1}(0) \cup a_{2}(0) \cup a_{1}(1 / 2) \cup a_{2}(1 / 2) \cup w_{1}(0) \cup w_{2}(0)\right) \\
& \quad=\# F(\delta) \cap\left(a_{1}(0) \cup a_{2}(0)\right)=\# \gamma \cap\left(a_{1}(0) \cup a_{2}(0)\right),
\end{aligned}
$$

we have $\delta \cap\left(a_{1}(1 / 2) \cup a_{2}(1 / 2) \cup w_{1}(0) \cup w_{2}(0)\right)=\emptyset$. Hence $\gamma$ can be chosen such that

$$
\gamma \cap\left(a_{1}(1 / 2) \cup a_{2}(1 / 2) \cup w_{1}(0) \cup w_{2}(0)\right)=\emptyset,
$$

and $\# \gamma \cap\left(a_{1}(0) \cup a_{2}(0)\right)$ is minimal. As a consequence,

$$
\delta \cap\left(a_{1}(1 / 2) \cup a_{2}(1 / 2) \cup w_{1}(0) \cup w_{2}(0)\right)=\emptyset,
$$

and $\# \delta \cap\left(a_{1}(0) \cup a_{2}(0)\right)$ is minimal.

Define by induction $\gamma_{k}$ to be the unique component of $F^{-k}(\gamma)$ homotopic to $\gamma$. Then for each $k$, the number $\# \gamma_{k} \cap\left(a_{1}(0) \cup a_{2}(0)\right)$ is minimal, and

$$
\gamma_{k} \cap\left(\bigcup_{j=1}^{k} F^{-j}\left(a_{1}(0) \cup a_{2}(0)\right)-a_{1}(0) \cup a_{2}(0)\right)=\emptyset .
$$

Let $l$ be the minimal integer such that $a_{1}\left(1 / 2^{l}\right)$ intersects $w_{2}\left(-1 / 2^{l-1}\right)$. Denote by $C$ the Jordan curve

$$
\begin{aligned}
a_{1}(1 / 2) \cup a_{1}\left(1 / 2^{l}\right) & \cup w_{2}\left(-1 / 2^{l-1}\right) \\
\cup & w_{2}(0) \cup w_{1}(0) \cup w_{1}\left(1 / 2^{l-1}\right) \cup a_{2}\left(-1 / 2^{l}\right) \cup a_{2}(1 / 2)
\end{aligned}
$$

in case $x_{0} \notin a_{1}\left(1 / 2^{l}\right)$ (which is the Jordan curve in the graph $g$, see Figure $4^{\prime}$ ), or the Jordan curve

$$
a_{1}(1 / 2) \cup a_{1}\left(1 / 2^{l}\right) \cup a_{2}\left(-1 / 2^{l}\right) \cup a_{2}(1 / 2),
$$


in case $l>1$ and $x_{0} \in a_{1}\left(1 / 2^{l}\right)$, or the arc $a_{1}(1 / 2) \cup a_{2}(1 / 2)$ in case $x_{0} \in$ $a_{1}(1 / 2)$.

In any case $\gamma_{l} \cap C=\emptyset$. So $\gamma_{l}$ is contained in one component of $S^{2}-C$. But each of these components contains at most one critical point of $F$. The curve $\gamma_{l}$ cannot separate $p_{1}, p_{2}, p_{3}$ and $x_{0}$ two by two. On the other hand, since $\gamma_{l-1}$ is homotopic to $\gamma$ rel $P_{F}$, by Theorem 3.3 each component of $S^{2}-\gamma_{l-1}$ contains exactly two points of the set $\left\{p_{1}, p_{2}, p_{3}, x_{1}\right\}$, hence each component of $S^{2}-\gamma_{l}$ must contain exactly two points of the set $\left\{p_{1}, p_{2}, p_{3}, x_{0}\right\}$. This is impossible.

Corollary 3.7. Assume in addition that the unique free critical point $x_{0}$ of $F$ is either periodic, or eventually falls into the set $\left\{p_{1}, p_{2}, p_{3}, \infty\right\}$. Then $F$ is equivalent to a Newton map.

Pr o of. We need to show that, under the extra hypothesis, the mapping $F$ cannot have a degenerate Levy cycle. Recall that a degenerate Levy cycle produces a cycle of disc-components $B_{1}, \ldots, B_{n}$ with $\# B_{i} \cap P_{F} \geq 2$, and each $B_{i}$ has a degree-one preimage $B_{i}^{\prime}$ isotopic to $B_{i-1}$ (with $B_{0}=B_{n}$ ), hence $\bigcup_{i} B_{i}$ contains at least one periodic cycle of $P_{F}$ without any critical points. This is impossible when $x_{0}$ is periodic or falls into $\left\{p_{1}, p_{2}, p_{3}, \infty\right\}$.

This corollary enables us to construct some Newton maps. Let $\alpha$ be a rational angle in $\mathcal{S}$, i.e. $2^{n} \alpha(\bmod 1) \subset[\alpha, 1]$ for all $n$. We can construct a branched covering $F$ modeled topologically by the description in Subsection 2.3. If $\alpha$ is dyadic, then $x_{0}$ coincides with the end point of $a_{1}(\alpha)$. If $\alpha$ is periodic of period $k$, we can modify $F$ in $U$ so that $F^{k}\left(x_{0}\right)=x_{0}$. In both cases $F$ is equivalent to a Newton map by the above corollary.

Remark 3.8. We would like to conclude at this point that

1. The Newton map $N$ equivalent to $F$ has Head angle $\alpha$. Unfortunately, we do not know how to check it directly.

2. If $\alpha$ is a rational element of $\mathcal{S}$ but not on the boundary of any connected component of $\mathbb{T}-\mathcal{S}$ (for example, $\alpha=1 / 6$ ), we can still construct a branched covering $F$ modeled topologically by Subsection 2.3. But we do not know whether $F$ has no degenerate Levy cycle.

In the next sections we will develop mating and capture techniques for the purpose of constructing all postcritically finite Newton maps. At the end of this paper, as a consequence of these more sophisticated results, we will be able to give positive answers to the above two questions.

4. Newton maps regarded as matings of polynomials. Mating is a way to construct branched coverings from pairs of polynomials. If a rational map is equivalent to a mating, we can get information about its dynamics from the information about those of the corresponding polynomials. In this 
section we use Theorem 3.3 to study the relationship between Newton maps and matings of cubic polynomials.

4.1. Definition of matings and general results. Let us recall the definitions of several kinds of matings, using the terminology of [ST], and state some general results.

DEFINITION 4.1 (formal mating). Let $f$ and $g$ be two monic polynomials of degree $d$. Set

$$
\widetilde{\mathbb{C}}=\mathbb{C} \cup\left\{\infty \cdot e^{2 \pi i \theta} \mid \theta \in \mathbb{T}=\mathbb{R} / \mathbb{Z}\right\}
$$

We can extend $f$ and $g$ continuously to $\widetilde{\mathbb{C}}$ by setting

$$
f\left(\infty \cdot e^{2 \pi i \theta}\right)=\infty \cdot e^{2 d \pi i \theta}, \quad g\left(\infty \cdot e^{2 \pi i \theta}\right)=\infty \cdot e^{2 d \pi i \theta}
$$

Let

$$
S_{f, g}^{2}=\widetilde{\mathbb{C}}_{f} \sqcup \widetilde{\mathbb{C}}_{g} /\left\{\left(\infty \cdot e^{2 \pi i \theta}, f\right) \approx\left(\infty \cdot e^{-2 \pi i \theta}, g\right)\right\}
$$

(where $\approx$ means identification). The formal mating of $f$ and $g$ is defined to be the branched covering $f \Perp g: S_{f, g}^{2} \rightarrow S_{f, g}^{2}$ such that

$$
f \Perp g=f \text { on } \widetilde{\mathbb{C}}_{f} \text { and } f \Perp g=g \text { on } \widetilde{\mathbb{C}}_{g} .
$$

The set $\left\{\left(\infty \cdot e^{2 \pi i \theta}, f\right) \mid \theta \in \mathbb{T}=\mathbb{R} / \mathbb{Z}\right\}$ in $S_{f, g}^{2}$ is called the equator of $f \Perp g$.

In case there is no ambiguity, we write $S^{2}$ instead of $S_{f, g}^{2}$.

From now on we assume that $f$ and $g$ are postcritically finite polynomials of the same degree and set $F=f \Perp g$. Then $F$ is also postcritically finite.

See [DH1] for the definitions of the filled Julia set $K_{f}$ for a polynomial $f$ and the external rays in $\mathbb{C}$. For $\theta \in \mathbb{T}$, denote by $R_{f}(\theta)$ the closure in $\widetilde{\mathbb{C}}_{f}$ of the external ray of angle $\theta$ (note that if $f$ is a postcritically finite polynomial, then $K_{f}$ is connected and locally connected, and $R_{f}(\theta)$ is well defined).

In $S_{f, g}^{2}$, the external rays $R_{f}(\theta)$ and $R_{g}(-\theta)$ are connected at the point $\left(\infty \cdot e^{2 \pi i \theta}, f\right)$.

Definition (ray-equivalence). For $x$ and $y$ in $\widetilde{\mathbb{C}}_{f}$, we define $x \sim_{f} y$ to be the equivalence relation generated by $x, y \in R_{f}(\theta)$ for some $\theta$. The equivalence relation $\sim_{g}$ on $\widetilde{\mathbb{C}}_{g}$ is similarly defined. In $S_{f, g}^{2}$, let $\sim_{F}$ be the equivalence relation generated by $\sim_{f}$ on $\widetilde{\mathbb{C}}_{f}$ and $\sim_{g}$ on $\widetilde{\mathbb{C}}_{g}$. These relations are called the ray-equivalence relations for $f$ and $g$ and $F$ resp. Denote by $[x]_{F}$ or simply $[x]$ the ray-equivalence class for $\sim_{F}$ of $x \in S^{2}$.

DEFINITION (matability). We say that two postcritically finite polynomials $f$ and $g$ are matable if $F=f \Perp g$ (or a modification of $F$ ) is Thurstonequivalent to a rational map (we have to modify $F$ slightly if there is a periodic tree-like ray-equivalence class $[x]$ with $\#[x] \cap P_{F} \geq 2$ ). 
DeFinition (topological mating). For the ray-equivalence relation $\sim_{F}$, denote by $\pi=\pi_{f, g}: S^{2} \rightarrow S^{2} / \sim_{F}$ the quotient projection, and by $F^{*}$ the quotient map of $F$. We say that $F^{*}$ is the topological mating of $f$ and $g$.

If we denote by $\gamma_{f}(\theta)$ (resp. $\gamma_{g}(\theta)$ ) the landing point of the external ray $R_{f}(\theta)$ (resp. $R_{g}(\theta)$ ). The quotient space $S^{2} / \sim_{F}$ is then homeomorphic to the quotient space

$$
K_{f} \sqcup K_{g} /\left\{\gamma_{f}(\theta) \approx \gamma_{g}(-\theta)\right\},
$$

i.e. the space obtained by gluing together the two filled Julia sets along their boundaries.

If two polynomials are matable, i.e. their formal mating is equivalent to some rational map $R$, then there is a constructive way to get a topological model of $R$, namely by topological mating. This is the following theorem of M. Rees:

THEOREM 4.1 ([R3]). Suppose that the formal mating $F$ of the two monic postcritically finite polynomials $g_{1}$ and $g_{2}$ is equivalent to a rational map $R$. Then there is a homeomorphism $\Phi$ from the quotient space $S^{2} / \sim_{F}$ to $\overline{\mathbb{C}}$ such that $\Phi \circ \pi$ is conformal on $\operatorname{int}\left(K_{g_{1}}\right) \cup \operatorname{int}\left(K_{g_{2}}\right)$, and that the following diagrams commute:

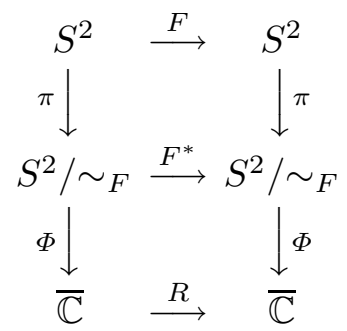

where $F^{*}$ is the topological mating of $g_{1}, g_{2}$. In particular, $F$ is semi-conjugate to $R$. Each fiber of the semi-conjugacy is a ray-equivalence class of $F$.

For a proof of the theorem, we suggest [Sh2].

By the theorem a necessary condition for two polynomials to be matable is that the quotient space $S^{2} / \sim_{F}$ is homeomorphic to the sphere. Although it is not in general a sufficient condition (as shown by the examples in [ST]), in some special cases it is: for example, in the quadratic case ([R3], [Ta]), or in the case that interests us in this work, as we are going to see in Corollary 4.5 and Theorem 4.6.

4.2. Matings which are equivalent to Newton maps. We study here the question whether the mating of two cubic polynomials is equivalent to a Newton map. We will do this by analyzing the ray-equivalence relation.

Denote by $\mathbf{f}$ the polynomial $z \mapsto z^{3}+(3 / 2) z$. It is the unique monic cubic polynomial (up to affine conjugacy) with two fixed critical points. These two fixed critical points are $u=i / \sqrt{2}$ and $v=-i / \sqrt{2}$. The point 0 is the unique 
repulsive fixed point of $\mathbf{f}$. The external 0-ray of $\mathbf{f}$ lands at this fixed point from the right side of the vector $\overrightarrow{v u}$, whereas the external 1/2-ray lands at it from the left side of $\overrightarrow{v u}$.

This polynomial will play a central role in our study of Newton maps. The Julia set of $\mathbf{f}$ is shown on the right of Figure 1.

If a cubic rational map with three distinct fixed critical points (i.e. a Newton map) is equivalent to the mating of two monic cubic polynomials $f_{1}$ and $f_{2}$, then one of the polynomials, say $f_{1}$, must have two fixed critical points, and is hence affine conjugate to $\mathbf{f}$, and $f_{2}$, the other, must have one fixed critical point.

Any cubic monic polynomial with one fixed critical point must be affine conjugate to the polynomial $g_{a}$, for some $a \in \mathbb{C}$, where

$$
g_{a}(z)=z^{3}-3 a^{2} z+2 a^{3}+a .
$$

The two critical points of $g_{a}$ are $a$ and $-a$. We have $g_{a}(a)=a$. We call $a$ the distinguished fixed critical point of $g_{a}$, and $-a$ the free critical point of $g_{a}$. Note that $g_{a}$ and $g_{-a}$ are conjugate by $\phi: z \mapsto-z$. Since $\phi \circ \mathbf{f} \circ \phi^{-1}=\mathbf{f}$, the two matings $\mathbf{f} \Perp g_{a}$ and $\mathbf{f} \Perp g_{-a}$ are topologically conjugate. To see the matability we only need to check one of them. The filled Julia set of $g_{a}$ is denoted by $K_{a}$ and its boundary by $J_{a}$. In case $K_{a}$ is locally connected (which is true if $g_{a}$ is postcritically finite, see [DH1], part I, pp. 24-29), we choose the $\varphi$-map $\varphi_{a}: \mathbb{C}-D \rightarrow \mathbb{C}-\operatorname{int}\left(K_{a}\right)$ such that $\varphi_{a}(z) / z \rightarrow 1$ as $z \rightarrow \infty$. Denote by $w$ the distinguished fixed critical point of $g_{a}$ (i.e. $w=a$ ). With these conventions the external 0-ray $\left\{\varphi_{a}(r) \mid 1 \leq r<\infty\right\}$ always lands at a point of the boundary of the immediate attracting basin of $w$. We thus get

Lemma 4.2. Any cubic mating $f_{1} \Perp f_{2}$ with three fixed critical points must be topologically conjugate to $\mathbf{f} \Perp g_{a}$ for $a \in \mathbb{C}$. The value of $a^{2}$ is unique for $f_{1} \Perp f_{2}$. If $\mathbf{f}$ and $g_{a}$ are matable, then $\mathbf{f} \Perp g_{a}$ is equivalent to a Newton map.

To study the ray-equivalence relation of $\mathbf{f} \Perp g_{a}$, we first state a general result about the Hubbard tree which is associated with each postcritically finite polynomial.

Every monic postcritically finite polynomial $g$ of degree $d$ has a forward invariant Hubbard tree $H_{g}$, which is the convex hull of $P_{g}$ in $K_{g}$. For example $H_{\mathbf{f}}$ for $\mathbf{f}$ is the segment connecting $i / \sqrt{2}$ and $-i / \sqrt{2}$. For a detailed description of Hubbard trees, we refer the reader to [DH1], I, pp. 30-36.

Proposition 4.3. Every point $x$ in the Julia set $J_{g}$ with more than one external ray eventually falls into the Hubbard tree $H_{g}$, under iterations by $g$.

Proof. Set $H=H_{g}, \beta=\gamma(0)$ the fixed point with external angle 0 ,

$$
\beta, \beta_{1}=\gamma\left(\frac{1}{d}\right), \beta_{2}=\gamma\left(\frac{2}{d}\right), \ldots, \beta_{d-1}=\gamma\left(\frac{d-1}{d}\right)
$$


the preimages of $\beta, H^{\prime}$ the "convex hull" of $\left\{\beta, \beta_{1}, \ldots, \beta_{d-1}\right\}$ in $K_{g}$ and $\widehat{H}$ the convex hull in $K_{g}$ of $P_{g} \cup\left\{\beta, \beta_{1}, \beta_{2}, \ldots, \beta_{d-1}\right\}$. According to DouadyHubbard's tree theory, $H, H^{\prime} \subset \widehat{H}$. Moreover, if $t$ and $t^{\prime}$, belonging to different components of

$$
[0,1]-\left\{\frac{1}{d}, \frac{2}{d}, \ldots, \frac{d-1}{d}\right\}
$$

are external angles of the same point $x$, then $x \in H^{\prime}$.

Suppose now that $x \in J_{g}$ and $x$ has at least two external angles $t, t^{\prime}$ $\left(t \neq t^{\prime}\right)$. Then in the expansions of $t, t^{\prime}$ in base $d$,

$$
t=0 . \varepsilon_{0} \varepsilon_{1} \ldots \varepsilon_{k} \ldots, \quad t^{\prime}=0 . \varepsilon_{0}^{\prime} \varepsilon_{1}^{\prime} \ldots \varepsilon_{k}^{\prime} \ldots,
$$

there is $k$ such that $\varepsilon_{k} \neq \varepsilon_{k}^{\prime}$ and hence $d^{k} t$ and $d^{k} t^{\prime}$ are in different components of (6) and thus $g^{k}(x) \in H^{\prime}$.

Now we need to show that every $x \in H^{\prime}$ having more than one external ray eventually falls into $H$. First of all, there is a unique point $a \in H$ such that $([\beta, a]-\{a\}) \cap H=\emptyset$, with $a=\beta$ if $\beta \in H$, where $[\beta, a]$ denotes the unique regular arc (see [DH1], I, p. 33 for definition) connecting $\beta$ and $a$. Since $[\beta, a]-\{a\}$ contains no critical values, we have $g\left(H^{\prime}\right) \subset H \cup[\beta, a]$.

If $\beta \in H$ then $a=\beta$ and $g\left(H^{\prime}\right) \subset H$, so we are done in this case.

Assume now $\beta \notin H$. Assume first that $[\beta, a]-\{a\}$ contains no critical points.

If $g(a) \neq a$, then $[\beta, a] \subset[\beta, g(a)],[a, g(a)] \subset H$ and $g:[\beta, a] \rightarrow[\beta, g(a)]$ is an expanding homeomorphism, so every point in $[\beta, a]-\{\beta\}$ eventually falls into $[a, g(a)] \subset H$ and then stays in $H$ since $g(H) \subset H$. If $g(a)=a$, let $a^{\prime}$ be the fixed point in $[\beta, a]$ closest to $\beta$. Then every point in $\left[\beta, a^{\prime}\right]-\{\beta\}$ is attracted to $a^{\prime}$. Hence $a^{\prime}$ is an attracting fixed point, which must be in $H$. So $a^{\prime}=a$. Therefore points in $[\beta, a]-\{\beta\}$ are contained in the attracting basin of $a$, hence disjoint from $J_{g}$. Consequently, $g\left(H^{\prime} \cap J_{g}\right) \subset H \cup\{\beta\}$.

Assume now $[\beta, a]-\{a\}$ contains critical points $c_{1}, \ldots, c_{l}$. They are ordered in the arc $[\beta, a]$ as $\beta<c_{1}<c_{2}<\ldots<c_{l}<a$. The map $g$ is injective in each of the subarcs. Since $g\left(c_{i}\right) \in H$, each of the subarcs, except $\left[\beta, c_{1}\right]$, is mapped homeomorphically onto an arc contained in $H$. Furthermore $g\left(\left[\beta, c_{1}\right]\right) \supset[\beta, a]$. For the same reason as above, there cannot be fixed points in $\left[\beta, c_{1}\right]-\{\beta\}$. So every point in $\left[\beta, c_{1}\right]-\{\beta\}$ is mapped eventually into $\left[c_{1}, a\right] \cup H$, and is mapped into $H$ after one more iteration.

Now let us examine the ray-equivalence relation of $F=\mathbf{f} \Perp g_{a}$. We will let $E$ denote the equator of $F$.

Lemma 4.4. Let $F=\mathbf{f} \Perp g_{a}$ with $g_{a}$ postcritically finite. There is an integer $K<\infty$ such that $\#[x] \cap E \leq K$ for each ray-equivalence class $[x]$. There is an equivalence class $[x]$ containing a closed loop if and only if $\gamma_{a}(0)=\gamma_{a}(1 / 2)$. None of the ray-equivalence classes contain more than one 
postcritical point. Furthermore, each ray-equivalence class contains at most one point of the Hubbard tree of $\mathbf{f}$, and at most one point of the Hubbard tree of $g_{a}$.

Pro of. According to Proposition 4.3 every point $x \in J_{a}$ (resp. $x \in J_{\mathbf{f}}$ ) with more than one external angle eventually falls into the Hubbard tree $H_{a}\left(\operatorname{resp} . H_{\mathbf{f}}\right)$.

The set $H_{\mathbf{f}} \cap J_{\mathbf{f}}$ is rather simple: it contains only one point $\beta=\gamma_{\mathbf{f}}(0)=$ $\gamma_{\mathbf{f}}(1 / 2)$ and $\beta$ has exactly two external angles 0 and $1 / 2$. Hence the rayequivalence relation $\sim_{\mathbf{f}}$ for $\mathbf{f}$ is also very simple: each equivalence class $[x]_{\mathbf{f}}$ contains at most two rays. Moreover, if $x \in J_{\mathrm{f}}$ has two external angles $\theta$, and $\theta^{\prime}$ then, by the above proposition, $x$ is a preimage of $\beta$, and $\theta$ and $\theta^{\prime}$ are preimages of 0 and $1 / 2$ under the mapping $\theta \mapsto 3 \theta$.

The ray-equivalence relation $\sim_{a}$ for $g_{a}$ is a little more complicated. Since $H_{a}$ is a topologically finite tree, it has a finite number of branch points, each with a finite number of branches. Denote by $k$ the maximal number of branches that a point of $H_{a}$ can have. According to Douady and Hubbard's counting-angle algorithm, we know that a point $x \in J_{a}$ has at most $2 k$ external angles (the free critical point of $g_{a}$ may double the maximal number of angles). Hence the equivalence class $[x]_{a}$ contains at most $2 k$ rays. Moreover, the point $\gamma_{a}(0)$ (resp. $\gamma_{a}(1 / 2)$ ) has either one or two rays: the 0 -ray and/or the $1 / 2$-ray.

To study $\sim_{F}$ we need some notation. For $x \in J_{\mathbf{f}} \sqcup J_{a}$ denote by $\operatorname{Ang}(x)$ the set of external angles of $x$. Denote by $R_{F}(\theta)$ the set $\overline{R_{\mathbf{f}}(\theta) \cup R_{a}(1-\theta)}$.

For any $[x]_{F}$ containing more than one ray we may suppose that there is $l \geq 0$ such that the intersection $\left[F^{l}(x)\right] \cap\left(H_{\mathbf{f}} \sqcup H_{a}\right)$ contains a point $y$ with more than one external angle, and these angles are either irrational or periodic under the mapping $\Theta_{3}: \mathbb{T} \rightarrow \mathbb{T}, \theta \mapsto 3 \theta$. We will show that there are only three topologically equivalent shapes for the set $\left[F^{l}(x)\right]=[y]$.

If $y \in H_{\mathbf{f}}$ then $[y]=R_{F}(0) \cup R_{F}(1 / 2)$. It is a closed curve (type 1 ) if $\gamma_{a}(0)=\gamma_{a}(1 / 2)$, and an arc consisting of two rays (type 2$)$ if not.

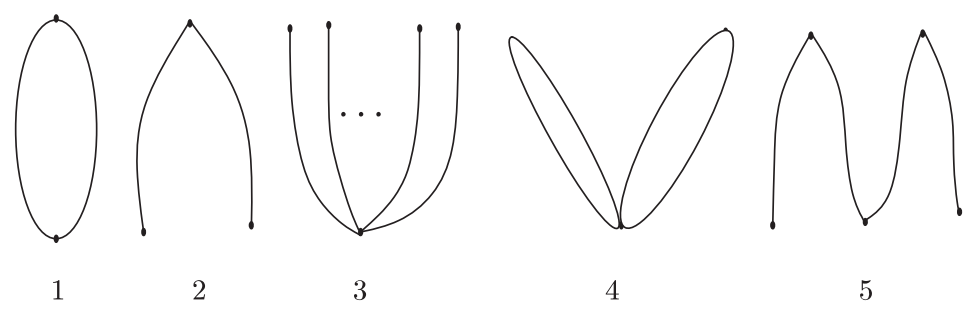

Fig. 8. Five possibilities of $[x]_{F}$

If $y \in H_{a}$ and $y=\gamma_{a}(0)$, then $[y]$ is of type 1 . 
If $y \in H_{a}$ and $y \neq \gamma_{a}(0)$, then, for each $\theta \in \operatorname{Ang}(y)$, the point $\gamma_{\mathbf{f}}(1-\theta)$ has only one ray, since $1-\theta$ is neither a preimage of 0 nor of $1 / 2$. Hence

$$
[y]=\bigcup_{\theta \in \operatorname{Ang}(y)} R_{F}(1-\theta) .
$$

It is a contractible graph, and $[y] \cap H_{a}=\{y\},[y] \cap H_{\mathbf{f}}=\emptyset$ (type 3 ), and $\#([y] \cap E) \leq k$.

Now for $[x]$. Denote by $z_{0}$ the free critical point of $g_{a}$ (i.e. $z_{0}=-a$ ). If $[x]$ does not contain a preimage of $z_{0}$ then it has exactly the same shape as $[y]$, since $F^{l}:[x] \rightarrow[y]$ is a homeomorphism. If $[x]$ does contain a preimage of $z_{0}$ then $F^{l}:[x] \rightarrow[y]$ is a double covering. If $[y]$ is of type 1 , then $[x]$ consists of two closed loops (four rays) connected at a point in $J_{a}$ (type 4). If $[y]$ is of type $2,[x]$ consists of four rays connected in a form of the letter $\mathrm{M}$, hence is contractible (type 5 ). If $[y]$ is of type 3 , then $[x]$ has almost the same shape as $[y]$ except

$$
\#([x] \cap E)=2 \#([y] \cap E) \leq 2 k
$$

(type 3).

The first assertion of the lemma is now proved by taking $K=\max \{4,2 k\}$. The second is also proved by checking each of the five possible types. For the third one, if $z_{0}$ is periodic, then each point of $P_{F}$ is in the interior of the two filled Julia sets, hence contains only itself in its $\sim_{F}$-class. If $z_{0}$ is strictly preperiodic, then $\left[z_{0}\right] \cap P_{F}=\emptyset$ since otherwise there is $i>0$ such that $F^{i}\left(z_{0}\right) \in\left[z_{0}\right]$ and there is $j \geq 0$ minimal such that $F^{j+i}\left(z_{0}\right)$ is periodic but $F^{j}\left(z_{0}\right)$ is not, and they both belong to $\left[F^{j}\left(z_{0}\right)\right]$. This is impossible. Therefore for each $i>0,\left[F^{i}\left(z_{0}\right)\right] \cap P_{F}=\left\{F^{i}\left(z_{0}\right)\right\}$. We are done.

The last statement of the lemma is a direct consequence of the above discussion.

The following result about matings of general polynomials can be easily proved using Moore's theorem (one can find a detailed proof in either [R3] or $[\mathrm{ST}])$ :

For $F=f \Perp g$ and $E$ the equator of $F$, if there is $K<\infty$ such that $\#[x]_{F} \cap E \leq K$ for each $x \in S_{f, g}^{2}$, and if none of the equivalence classes $[x]_{F}$ contains a closed loop, then $S^{2} / \sim_{F}$ is homeomorphic to $S^{2}$. We write $S^{2} / \sim_{F} \approx S^{2}$.

As a consequence of the above observation and Lemma 4.4 we have

Corollary 4.5. Let $F=\mathbf{f} \Perp g_{a}$ with $g_{a}$ postcritically finite. Then $S^{2} / \sim_{F} \approx S^{2}$ iff $\gamma_{a}(0) \neq \gamma_{a}(1 / 2)$.

We are now ready to give the mating criterion:

TheOREM 4.6. Let $F=\mathbf{f} \Perp g_{a}$ with $g_{a}$ postcritically finite. The two polynomials $\mathbf{f}$ and $g_{a}$ are matable if and only if $\gamma_{a}(0) \neq \gamma_{a}(1 / 2)$ (hence if 
and only if $S^{2} / \sim_{F} \approx S^{2}$ ). Or equivalently, $\mathbf{f}$ and $g_{a}$ are not matable iff $\gamma_{a}(0)=\gamma_{a}(1 / 2)$.

Remark. The location of values of parameters $a \in \mathbb{C}$ with $\gamma_{a}(0)=$ $\gamma_{a}(1 / 2)$ can be determined easily, and will be described in Section 5. For instance, if $\operatorname{Re}(a)=0, a \neq 0$, and $g_{a}$ is postcritically finite, then $\gamma_{a}(0)=$ $\gamma_{a}(1 / 2)$.

P r o of (of Theorem 4.6). By definition of matability we have to check when $F$, or a modification of $F$, is equivalent to a rational map. Lemma 4.4 asserts that no ray-equivalence class contains more than one point of $P_{F}$. So no modification is necessary. Since $F$ has three fixed critical points, it has a hyperbolic orbifold and we can apply Thurston's criterion (Theorem 3.1) to it and claim that $F$ is not equivalent to a rational map iff it has a Thurston obstruction.

If $\gamma_{a}(0)=\gamma_{a}(1 / 2)$, then in $S_{\mathbf{f}, g_{a}}^{2}$ the set $R_{\mathbf{f}}(0) \cup R_{\mathbf{f}}(1 / 2) \cup R_{a}(0) \cup$ $R_{a}(1 / 2)$ forms a Levy cycle for $F$. This Levy cycle will generate a Thurston obstruction for $F$ (Theorem 3.1).

Suppose now that $F$ has a minimal Thurston obstruction $\Gamma$. A convergence theorem stated in $[\mathrm{Ta}]$ (Proposition 2.7, p. 597) shows that the pull backs of any degenerate Levy cycle will converge to a periodic cycle of ray-classes, each containing at least two postcritical points. Combining this with Lemma 4.4 we conclude that $F$ cannot have a degenerate Levy cycle. Applying Theorem 3.3 to $\Gamma$, one can see that $\Gamma$ is decomposed into $\{\gamma\} \cup \Gamma_{2}$ with $\Gamma_{1}=\{\gamma\}$ a Levy cycle. By the same convergence theorem stated in [Ta], the pull backs of $\gamma$ will converge to a closed curve contained in a periodic ray-equivalence class. The only possibility is then (Lemma 4.4) $\gamma_{a}(0)=\gamma_{a}(1 / 2)$, and $R_{a}(0) \cup R_{a}(1 / 2)$ together with $R_{\mathbf{f}}(0) \cup R_{\mathbf{f}}(1 / 2)$ gives the closed curve.

4.3. From polynomials to Newton maps via matings. Now we ask the converse question: when is a postcritically finite Newton map $N$ equivalent to the mating of two cubic polynomials? The answer is quite simple, namely, when $p_{3} \notin \operatorname{Or}\left(x_{0}\right)$.

Let us recall some results of Section 2.1. The three fixed critical points of $N$ are labeled $p_{1}, p_{2}$ and $p_{3}$. The free critical point is denoted by $x_{0}$. The unique non-attracting fixed point is normalized to be $\infty$. The attracting basin of $p_{i}$ is denoted by $A_{i}$. Assume $x_{0} \notin A_{1} \cup A_{2} \cup A_{3}$. Denote by $W_{i}$ the component of $N^{-1}\left(A_{i}\right)$ disjoint from $A_{i}, i=1,2,3$. The three 0-rays $a_{1}(0)$, $a_{2}(0)$ and $a_{3}(0)$ are connected at $\infty$ in counterclockwise order. Moreover, $N^{-1}(\infty)=\left\{\infty, \xi_{1}, \xi_{2}\right\}$ with

$$
\xi_{1} \in \partial A_{1} \cap \partial A_{2} \cap \partial W_{3} .
$$


We have $\# \partial A_{1} \cap \partial A_{2}=\infty$ and $\bar{A}_{3} \cap\left(\bar{A}_{1} \cup \bar{A}_{2}\right)=\{\infty\}$ when $\xi_{1} \neq \xi_{2}$. Set $x_{i}=N^{i}\left(x_{0}\right), \operatorname{Or}\left(x_{0}\right)=\left\{x_{i} \mid i \geq 0\right\}$.

Recall from Section 4.2 that $u=i / \sqrt{2}, v=-i / \sqrt{2}$ denote the two critical points of $\mathbf{f}$, so that the external 0-ray of $\mathbf{f}$ comes from the right side of the vector $\overrightarrow{v u}$. Denote by $w$ the distinguished fixed critical point of $g_{a}$, by $z_{0}$ the other critical point, by $K_{a}$ the filled Julia set of $g_{a}$ and by $H_{a}$ its Hubbard tree. Denote by $A_{u}, A_{v}, A_{w}, \varphi_{u}, \varphi_{v}, \varphi_{w}, R_{u}(t), R_{v}(t), R_{w}(t)$ the corresponding immediate basin, the linearization mapping, and the internal $\ell$-ray of $u, v, w$, respectively. Recall that the external 0-ray of $g_{a}$ lands at a point of $\bar{A}_{w}$. Except in the case where $R_{F}(1 / 3)$ and $R_{F}(2 / 3)$ both land at the same point (which must be $z_{0}$ ), only one of $R_{F}(1 / 3)$ and $R_{F}(2 / 3)$ lands at a point of $\partial A_{w}$ (since $g_{a}: \partial A_{w} \rightarrow \partial A_{w}$ is a degree two covering).

Denote by $[N]$ the conformal conjugacy class of $N$. Note that the property $\left\{p_{2}, p_{3}\right\} \cap \operatorname{Or}\left(x_{0}\right)=\emptyset$ is preserved by conjugacy.

THEOREM 4.7. There is a well defined surjective mapping $m_{1}$ from

$$
\begin{aligned}
& A=\left\{a \mid \operatorname{Re}(a)>0, g_{a}\right. \text { is postcritically finite, } \\
& \left.\qquad \gamma_{a}(0) \neq \gamma_{a}(1 / 2), \gamma_{a}(2 / 3) \in \partial A_{w}\right\}
\end{aligned}
$$

onto

$$
\left\{[N] \mid N \text { is postcritically finite, } x_{0} \notin A_{1} \cup A_{2} \cup A_{3},\right.
$$

$$
\left.\left\{p_{2}, p_{3}\right\} \cap \operatorname{Or}\left(x_{0}\right)=\emptyset\right\}
$$

satisfying: for $[N]=m_{1}(a)$, and any $N$ in the conjugacy class, there is a mapping $\phi: S^{2} \rightarrow \overline{\mathbb{C}}$ with the following properties:

1. $\phi$ is continuous.

2. For any $z \in \overline{\mathbb{C}}$, the set $\phi^{-1}(z)$ is a ray-equivalence class of $\mathbf{f} \Perp g_{a}$.

3. $\phi \circ\left(\mathbf{f} \Perp g_{a}\right)=N \circ \phi$, and $\phi$ is conformal in $\operatorname{int}\left(K_{\mathbf{f}}\right) \cup \operatorname{int}\left(K_{a}\right)$.

4. $\phi(w)=p_{1}$.

Furthermore $\# m_{1}^{-1}([N])=2$ if $\infty \in \operatorname{Or}\left(x_{0}\right)$ and $\# m_{1}^{-1}([N])=1$ otherwise. Similarly if we switch $p_{1}$ and $p_{2}$, and change $2 / 3$ to $1 / 3$, we can get another mapping $m_{2}$ with the same properties.

Corollary 4.8. A postcritically finite Newton map $N$ is equivalent to a mating if and only if

$$
\operatorname{Or}\left(x_{0}\right) \not \supset p_{3} .
$$

R e $\mathrm{m}$ ark. Since $\mathbf{f} \Perp g_{0}$ is equivalent to $\mathbf{f}$, one can naturally extend $m_{1}$ by setting $m_{1}(0)=[\mathbf{f}]$.

Pro of (of Theorem 4.7). Take a point $a \in A$. The two polynomials $\mathbf{f}$ and $g_{a}$ are matable by Theorem 4.6. Thus $\mathbf{f} \Perp g_{a}$ is equivalent to a rational map $N$, which is certainly a Newton map. Set $F=\mathbf{f} \Perp g_{a}$. According to Theorem 4.1, there is a continuous semi-conjugacy $\phi=\Phi \circ \pi: S^{2} \rightarrow \overline{\mathbb{C}}$ from 
$F$ to $N$. The mapping $\phi$ is conformal $\operatorname{in} \operatorname{int}\left(K_{\mathbf{f}}\right) \cup \operatorname{int}\left(K_{a}\right)$. Each fiber of $\phi$ is a ray-equivalence class of $F$. Now the condition $\gamma_{a}(2 / 3) \in \partial A_{w}$ shows that one end of $R_{F}(1 / 3)$ lands at a point of $\partial A_{u}$ and the other end lands at a point of $\partial A_{w}$. So $\phi\left(\partial A_{u}\right)$ and $\phi\left(\partial A_{w}\right)$ have at least two common points, $\infty$ and $\phi\left(R_{F}(1 / 3)\right)$. Thus $\phi\left(A_{u}\right) \cup \phi\left(A_{w}\right)$ must be $A_{1} \cup A_{2}$. Considering the cyclic order at $\infty$, we can then determine that $\phi(u)=p_{2}, \phi(v)=p_{3}$, and $\phi(w)=p_{1}$.

Since $\operatorname{Or}\left(x_{0}\right)=\phi\left(\operatorname{Or}\left(z_{0}\right)\right) \subset \phi\left(K_{a}\right)$, we have $\left\{p_{2}, p_{3}\right\} \cap \operatorname{Or}\left(x_{0}\right)=\emptyset$. That is, $[N] \in(8)$.

Set $m_{1}(a)=[N]$. The mapping $m_{1}$ is then well defined from $A$ into the set (8).

To get the surjectivity of $m_{1}$, we need to apply the following ThurstonLevy mating criterion ([Th], [Le], [W]):

Let $F$ be a postcritically finite branched covering of degree $d$. Assume that $F$ has no degenerate Levy cycle. Then $F$ is equivalent to the mating of two polynomials $f$ and $g$ if and only if there is a closed curve $\gamma \subset S^{2}-P_{F}$ such that $F^{-1}(\gamma)=\gamma^{\prime}$ is again a single closed curve and $\gamma^{\prime}$ is homotopic to $\gamma$ rel $P_{F}$ and has the same orientation. Moreover, given such a $\gamma$, the two polynomials $f$ and $g$ are uniquely determined, up to the symmetric conjugacy: $\psi_{j} \circ f \circ \psi_{j}^{-1}$ and $\psi_{j}^{-1} \circ g \circ \psi_{j}$, where $\psi_{j}: z \mapsto e^{2 \pi i j / d} z, j=$ $1, \ldots, d-1$.

Let us get back to the proof of Theorem 4.7. Take $[N]$ in (8), and $N$ a representative of the class. Set

$$
\ell=\left\{p_{2}\right\} \cup a_{2}(0) \cup\left\{p_{3}\right\} \cup a_{3}(0) \cup\{\infty\} .
$$

Suppose first that $\infty \notin \operatorname{Or}\left(x_{0}\right)$. Then $\ell \cap P_{N}=\left\{p_{2}, p_{3}\right\}$ (where $P_{N}$ denotes the postcritical set of $N$ ), and $N^{-1}(\ell)$ is a connected arc homotopic to $\ell$ rel $P_{N}$. A curve $\gamma$ around $\ell$ in a small neighborhood of $\ell$ satisfies the condition of the above mating criterion, and will give a uniquely determined value $a^{2}$ such that $N \sim \mathbf{f} \Perp g_{a}$. We have $\gamma_{a}(0) \neq \gamma_{a}(1 / 2)$ according to Theorem 4.6. The mapping $\phi$ is also well defined, satisfying the four properties required. It is easy to show that $\gamma_{a}(2 / 3) \in \partial A_{w}$. Since $\mathbf{f} \Perp g_{a} \sim \mathbf{f} \Perp g_{-a}$ (see the paragraph preceding Lemma 4.2), we may choose $a$ such that $\operatorname{Re}(a) \geq 0$.

On the other hand, for $a \in i \mathbb{R}$, one can use the fact that $g_{a}$ preserves the imaginary axis to check that either $\gamma_{a}(0)=\gamma_{a}(1 / 2)$, or the free critical point of $g_{a}$ is in the basin of the fixed critical point. In other words, there is no postcritically finite $g_{a}$ with $\operatorname{Re}(a)=0, a \neq 0$ and $\gamma_{a}(0) \neq \gamma_{a}(1 / 2)$.

Thus one can choose $a$ with $\operatorname{Re}(a)>0$ such that $N \sim \mathbf{f} \Perp g_{a}$. Therefore $[N]=m_{1}(a)$ with $a \in A$.

Suppose now $\infty \in \operatorname{Or}\left(x_{0}\right)$. There are exactly two non-homotopic ways $\left(\right.$ rel $P_{N}$ ) to modify $\ell$ in a small neighborhood of $\infty$ in order to avoid $\infty$. Applying the mating criterion to them, we get two values $a, a^{\prime} \in A$ such that $N \sim \mathbf{f} \Perp g_{a} \sim \mathbf{f} \Perp g_{a^{\prime}}$. Thus $m_{1}(a)=m_{1}\left(a^{\prime}\right)=[N]$. 
To see that $a \neq a^{\prime}$, let $k$ be the minimal integer such that $N^{k}\left(x_{0}\right)=\infty$. Then $g_{a}^{k}\left(z_{0}\right)$ is the landing point of the external 0-ray whereas $g_{a^{\prime}}^{k}\left(z_{0}\right)$ is the landing point of the external 1/2-ray (or vice versa). Thus the dynamics of $z_{0}$ under $g_{a}$ and $g_{a^{\prime}}$ are not conjugate. We are done.

Finally, we should check that there are no other values in $m_{1}^{-1}([N])$. Suppose $a \in A$ such that $\mathbf{f} \Perp g_{a} \sim N$, and the semi-conjugacy $\phi$ maps $w$ to $p_{1}$. Then the Hubbard tree $H_{\mathbf{f}}$ of $\mathbf{f}$ must be projected by $\phi$ to $\ell$ (the mapping $\phi$ maps the $t$-ray of $A_{u}$ to the $t$-ray of $A_{2}$, etc.). The rest follows from the uniqueness described in the mating criterion.

EXAmples. 1. $N_{P}$ for $P: z \mapsto z^{3}-z$ is equivalent to $\mathbf{f} \Perp g_{0}$, since $a=0$ is the only point in $\mathbb{C}$ such that $g_{a}$ has a double critical point.

2. $N_{P}$ for $P: z \mapsto z^{3}-1$ is equivalent to $\mathbf{f} \Perp g_{1 / 2}$, since $\pm 1 / 2$ are the only values of $a \in \mathbb{C}$ such that one critical point of $g_{a}$ has a repelling fixed point as its image.

5.Newton maps regarded as captures from $K_{\mathrm{f}}$. Capture is a way to construct branched coverings from a single polynomial. In this section we will describe all Newton maps which are equivalent to a capture. Part of the work was done by J. Head. The rest is a consequence of our results about matings.

Recall that $\mathbf{f}$ denotes the polynomial $z \mapsto z^{3}+(3 / 2) z$, and $u=i / \sqrt{2}$ and $v=-i / \sqrt{2}$ are the two fixed critical points of $\mathbf{f}$. There are two ways to define external rays on $\mathbb{C}-K_{\mathbf{f}}$. We choose the one such that the 0-ray comes from the right side of the vector $\overrightarrow{v u}$. Denote by $R_{\mathbf{f}}(\theta)$ the external ray of angle $\theta$, and by $\gamma_{\mathbf{f}}(\theta)$ its landing point on $\partial K_{\mathbf{f}}$. Denote by $A_{u}$ the immediate attracting basin of $u$. The mapping $\left.\mathbf{f}\right|_{A_{u}}$ is conjugate to $z \mapsto z^{2}$. Denote by $R_{u}(t)$ the corresponding internal $t$-ray. The set of connected components of $K_{\mathbf{f}}-\bar{A}_{u}$ can be parametrized by the internal angle $t$ of the attaching point to $\bar{A}_{u}$ of that conected component. It is called the $t$-limb. The angle $t$ is dyadic. If $t \neq 0$, the mapping $\mathbf{f}$ carries the $t$-limb homeomorphically to the $2 t(\bmod 1)-\operatorname{limb}$. The critical point $v$ is contained in the 0 -limb.

Denote by $\xi$ the preimage of 0 on $\partial A_{u}$ with internal angle $1 / 2$. Note that the three rays $R_{u}(1 / 2), R_{\mathbf{f}}(1 / 3)$ and $R_{\mathbf{f}}(1 / 6)$ all land at $\xi$, and the two rays $R_{u}(0)$ and $R_{\mathbf{f}}(0)$ both land at 0 , the unique repelling fixed point of $\mathbf{f}$.

Notation. Denote by $\mathcal{Y}$ the intersection of $K_{\mathrm{f}}$ with the closed region not containing $v$ bounded by the curve $R_{u}(0) \cup R_{u}(1 / 2) \cup R_{\mathbf{f}}(1 / 6) \cup R_{\mathbf{f}}(0) \cup\{\infty\}$. For any $y \in \mathcal{Y}-\left\{0, \gamma_{\mathbf{f}}(1 / 6)\right\}$, denote by $y_{0}$ and $y_{0}^{\prime}$ the two points in $K_{\mathbf{f}}$ distinct from $y$ such that $\mathbf{f}\left(y_{0}\right)=\mathbf{f}\left(y_{0}^{\prime}\right)=\mathbf{f}(y)$. Denote by $Y$ the subset of $\mathcal{Y}-\left\{0, \gamma_{\mathbf{f}}(1 / 6)\right\}$ consisting of (pre)periodic points $y$ such that neither $y_{0}$ nor $y_{0}^{\prime}$ is periodic.

Let $y \in Y$ and set $y_{k}=\mathbf{f}^{k}\left(y_{0}\right)$. There are then minimal integers $l \geq 1$ and $p \geq 1$ such that $y_{l+p}=y_{l}$. Just as in the construction of Hubbard trees, there 
is a unique convex hull $T$ in $K_{\mathbf{f}}$ of the finite set $\left\{u, v, \xi, y_{0}^{\prime}, y_{0}, y_{1}, \ldots, y_{l+p-1}\right\}$. Set $T(y)=T \cup R_{\mathbf{f}}(0) \cup R_{\mathbf{f}}(1 / 3) \cup\{\infty\}$. It has the following properties:

1. $T(y)$ is a topological finite graph.

2. $T(y)$ contains the finite set $\left\{\infty, u, v, \xi, y_{0}^{\prime}, y_{0}, y_{1}, \ldots, y_{l+p-1}\right\}$.

3. The extremities of $T(y)$ are contained in $\left\{v, y_{0}^{\prime}, y_{0}, y_{1}, \ldots, y_{l+p-1}\right\}$. The two points $y_{0}, y_{0}^{\prime}$ are extremities of $T(y)$.

4. $T(y)$ is forward invariant under f i.e. $\mathbf{f}(T(y)) \subset T(y)$.

5. The polynomial $\mathbf{f}$ is injective on each edge of $T(y)$.

6 . The local degree of $\mathbf{f}$ as a graph-mapping is 2 at $\infty, u$ and $v$, and 1 at any other vertex of $T(y)$.

7. If $y \neq y^{\prime}$, then $T(y)$ and $T\left(y^{\prime}\right)$ do not coincide.

For each $y \in Y$, the next lemma establishes the existence and uniqueness of a cubic branched covering with a specified dynamics. It is essentially due to J. Head.

Lemma 5.1 (Head). For any point $y$ in $Y$, there is a cubic branched covering $F=F_{y}$ of $S^{2}$ with the following properties: $F$ has four critical points $x_{0}, p_{1}, p_{2}$ and $p_{3}$, where $p_{1}, p_{2}$ and $p_{3}$ are also fixed points and labeled in the way such that their immediate basins $A_{1}, A_{2}$ and $A_{3}$ are connected at $\infty$ in counterclockwise order, and that $\partial A_{1} \cap \partial A_{2}$ contains more than one point. There is a mapping $\varphi$ from $T(y)$ to $S^{2}$, satisfying

1. $\varphi$ is continuous and injective on $T(y)-\left\{y_{0}^{\prime}\right\}$, with $\varphi\left(y_{0}\right)=\varphi\left(y_{0}^{\prime}\right)=x_{0}$.

2. $\varphi$ preserves the cyclic order around each branch point.

3. $\varphi(\infty)=p_{1}, \varphi(u)=p_{2}$ and $\varphi(v)=p_{3}$.

4. $\left.\varphi \circ \mathbf{f}\right|_{T(y)}=\left.F \circ \varphi\right|_{T(y)}$.

Moreover, any cubic branched covering which satisfies the above conditions is Thurston-equivalent to $F_{y}$. Any such branched covering is called a capture of $\mathbf{f}$ with co-critical point at $y$.

Proof. For the construction of $F_{y}$, we will follow the procedure given by J. Head ([He]) except that we replace her abstract tree by $T(y)$.

Take an embedding $\varphi$ of $T(y) /\left\{y_{0} \sim y_{0}^{\prime}\right\}$ to $S^{2}$ such that $\varphi(0)=\infty$ and $\varphi$ preserves the cyclic order at branch points. The homotopy type of $\varphi$ is unique. Write $\varphi(\infty)=p_{1}, \varphi(u)=p_{2}, \varphi(v)=p_{3}, \varphi\left(y_{0}\right)=\varphi\left(y_{0}^{\prime}\right)=x_{0}$ and $\varphi\left(T(y) /\left\{y_{0} \sim y_{0}^{\prime}\right\}\right)=G$. Note that $S^{2}-G$ has three connected components, each homeomorphic to a disc.

Let us construct a cubic branched covering $F=F_{y}: S^{2} \rightarrow S^{2}$. Set $\left.F\right|_{G}=\varphi \circ \mathbf{f} \circ \varphi^{-1}$. For each connected component $U$ of $S^{2}-G, F(\partial U)$ is a subtree of $G$ so $S^{2}-F(\partial U)$ is homeomorphic to an open disc. By Schoenflies' theorem, there is a homeomorphic extension of $F$ from $\bar{U}$ to $S^{2}$. Take an extension of $F$ for each connected component of $S^{2}-G$. This gives a cubic branched covering. The critical points are $x_{0}, p_{1}, p_{2}$ and $p_{3}$. We are done. 
The uniqueness of $F_{y}$ up to Thurston-equivalence is proved by J. Head in Proposition 4.3 .1 of [He], where she uses a more general result of B. Wittner ([W], Theorem 4.4.8). We will skip the proof here.

Recall that a Newton map is a cubic rational map with three fixed critical points $p_{1}, p_{2}$ and $p_{3}$, labeled in the way that their immediate attracting basins are attached at a common fixed point in counterclockwise order, and that the immediate basins of $p_{1}, p_{2}$ have more than one common point on the boundary. Up to conformal conjugacy, we can assume that the point $\infty$ in $\overline{\mathbb{C}}$ is the unique non-critical fixed point. Denote as usual by $x_{0}$ the unique free critical point, and by $[N]$ the conformal conjugacy class of $N$.

THEOREM 5.2. There is a well defined surjective mapping $c_{2}$ (capture) from $Y \cup\{u\}$ onto the set

$$
\begin{aligned}
& \{[N] \mid N \text { is postcritically finite, } \\
& \left.\qquad x_{0} \text { is neither periodic nor a preimage of } p_{1}\right\}
\end{aligned}
$$

such that for $[N]=c_{2}(y)$, there is a mapping $\phi: T(y) \rightarrow \overline{\mathbb{C}}$ with the following properties:

1. $\phi$ is continuous and injective on $T(y)-\left\{y_{0}^{\prime}\right\}$, with $\phi\left(y_{0}\right)=\phi\left(y_{0}^{\prime}\right)=x_{0}$.

2. $\phi$ preserves the cyclic order around each branch point.

3. $\phi(\infty)=p_{1}, \phi(u)=p_{2}$ and $\phi(v)=p_{3}$.

4. $\left.\phi \circ \mathbf{f}\right|_{T(y)}=\left.N \circ \phi\right|_{T(y)}$.

Moreover, $\# c_{2}^{-1}([N])=1$ if $\left\{p_{2}, p_{3}, \infty\right\} \cap \operatorname{Or}\left(x_{0}\right) \neq \emptyset$.

Proof. First set $c_{2}(u)=[\mathbf{f}]$.

We prove that for each $y \in Y$, the branched covering $F_{y}$ is Thurstonequivalent to a Newton map $N$, with $[N]$ in (10).

We decompose $Y$ into four disjoint subsets:

$Y_{\infty}=\left\{y \in Y \mid y_{0}\right.$ is a preimage of 0 , the unique non-critical fixed point $\}$. $Y_{\mathrm{sp}}=\left\{y \in Y \mid y_{0}\right.$ is strictly preperiodic, but not eventually fixed $\}$.

$Y_{2}=\left\{y \in Y \mid y_{0}\right.$ is a preimage of $\left.u\right\}$.

$Y_{3}=\left\{y \in Y \mid y_{0}\right.$ is a preimage of $\left.v\right\}$.

The case $y \in Y_{2} \cup Y_{3} \cup Y_{\infty}$ was done by J. Head ([He]), with a proof similar to that of our Theorem 3.6. The idea is that if $F=F_{y}$ has a one curve Levy cycle $\{\gamma\}$, then

$$
\gamma \cap\left(\overrightarrow{p_{1} \xi} \cup \xi \overrightarrow{p_{2}} \cup p_{2} \vec{x}_{0} \cup x_{0} \vec{p}_{3}\right)=\emptyset .
$$

So $\gamma$ cannot separate the four critical points of $F$ two by two. This contradicts Theorem 3.3. Hence $F$ has no non-degenerate Levy cycle. But $F$ cannot have any degenerate Levy cycle either. Recall that, by definition 
(Section 3), a degenerate Levy cycle must decompose $S^{2}$ into $n \geq 2$ disccomponents $B_{1}, \ldots, B_{n}$, and zero or one non-disc component. Moreover, $F\left(P_{F} \cap B_{i}\right)=P_{F} \cap B_{i+1(\bmod n)}$. So each $P_{F} \cap B_{i}$ contains only periodic points. By definition, the set $\partial B_{i}$ should be non-peripheral rel $P_{F}$, so $P_{F} \cap B_{i}$ contains at least two points, each of them being periodic of period at least 2 (note that $n \geq 2$ ). However, if $x_{0}$ eventually falls into $\left\{p_{1}, p_{2}, \infty\right\}$, then the postcritical set $P_{F}$ does not contain any non-fixed periodic point.

Thus $F$ is equivalent to a Newton map $N$. Moreover, because of the dynamical numbering of the critical points, the point $p_{i}$ of $F$ must correspond to $p_{i}$ of $N, i=1,2,3$. So $[N]$ is in (10).

To find the mapping $\phi$, we start from $N$. Denote by $a_{i}(0)$ the 0 -ray of $A_{i}$ as usual. Given any Newton map $N$ such that $N^{k}\left(x_{0}\right) \in\left\{p_{2}, p_{3}\right\}$ for some minimal integer $k>0$, in the connected graph $N^{-k}\left(\bar{a}_{2}(0) \cup \bar{a}_{3}(0)\right)$ one can find a minimal invariant graph $T$ such that $T$ contains the whole postcritical set $P_{N}$ except for $p_{1}$, and $T-\left\{x_{0}\right\}$ is a topologically finite tree. It is easy to find $z \in Y$ such that there is a homeomorphism $\phi$ from $T(z) /\left\{z_{0} \sim z_{0}^{\prime}\right\}$ to $T$, preserving the cyclic order at each branch point, mapping $\infty$ to $p_{1}, u$ to $p_{2}$, and $v$ to $p_{3}$, and satisfying $\phi \circ \mathbf{f}=N \circ \phi$. By Lemma 5.1, the rational map $N$ is Thurston-equivalent to $F_{z}$. Since $N \sim F_{y}$, we have $y=z$.

For $y \in Y_{\mathrm{sp}}$, the same argument shows that $F_{y}$ cannot have a nondegenerate Levy cycle. But it does not indicate whether $F$ has a degenerate Levy cycle. We will take another route, using an argument that works for $y \in Y_{\infty}$ as well.

We will see that for any $y \in Y_{\mathrm{sp}} \cup Y_{\infty}$, there is a point $a \in \mathbb{C}$ such that $F_{y}$ is equivalent to the mating $\mathbf{f} \Perp g_{a}$. Moreover, $\mathbf{f} \Perp g_{a}$ is equivalent to a Newton map.

Note that $Y_{\mathrm{sp}} \cup Y_{\infty} \subset \partial K_{\mathbf{f}}$. Let $y \in Y_{\mathrm{sp}} \cup Y_{\infty}$. Let $\theta$ be one external angle of $y$. An easy calculation shows that $\theta \in] 0,1 / 6[$ (by taking account of the internal angle of $y$ relative to $u$ ). Choose $a \in \mathbb{C}$ such that $1-\theta$ is one of the external angles of the co-critical point (i.e. the point $2 a$ ) of $g_{a}$. It is not difficult to see that such a value $a$ exists and is unique and, because $1-\theta \in] 5 / 6,1\left[\right.$, we have $\gamma_{a}(0) \neq \gamma_{a}(1 / 2)$ (where $\gamma_{a}(\eta)$ denotes the landing point of the external $\eta$-ray of $g_{a}$; we refer the reader to [M1] and [F] for details). The topological mating of $\mathbf{f}$ and $g_{a}$ gives a branched covering $F^{\prime}$ with the properties stated in Lemma 5.1. By uniqueness $F^{\prime}$ is Thurstonequivalent to $F_{y}$. Since $\mathbf{f}$ and $g_{a}$ are matable (Theorem 4.6), $F^{\prime}$ is equivalent to a Newton map. So is $F_{y}$.

Now the semi-conjugacy $\phi$ given by Theorem 4.7 will be injective on $T(y)-\left\{y_{0}^{\prime}\right\}$ since no pair of its points are in the same ray-equivalence class of $\mathbf{f} \Perp g_{a}$.

It is quite easy to see that $[N]$ belongs to the set (10). 
Now set $c_{2}(y)=[N]$. The mapping $c_{2}$ is then well defined from $Y$ into (10).

We now consider the surjectivity of $c_{2}$. Take $N$ with its class in (10). If $x_{0}$ coincides with one of the roots, then the map $N$ is in the conformal class $[\mathbf{f}]=c_{2}(u)$. We may now assume $x_{0} \notin\left\{p_{1}, p_{2}, p_{3}\right\}$. We will find $y \in Y$ such that $N$ is equivalent to $F_{y}$.

The case where $x_{0}$ is a preimage of $p_{2}$ or $p_{3}$ follows from the above construction of $\phi$.

For the other cases $x_{0}$ must be contained in the Julia set $J_{N}$ and is strictly preperiodic. We again use matings to construct $T(y)$. The mapping $N$ is topologically conjugate to the topological mating of $\mathbf{f}$ and $g_{a}$ for some $a \in \mathbb{C}$. The free critical point of $g_{a}$ is contained in the Julia set of $g_{a}$. Let $\theta$ be an external angle of the co-critical point. By our choice of $p_{2}$ and $p_{3}$ we have $\left.\theta \in\right] 5 / 6,1\left[\right.$. Denote by $y$ the point in $K_{\mathbf{f}}$ with external angle $1-\theta$. Then $y \in Y$ and $T(y)-\left\{y_{0}^{\prime}\right\}$ is embedded in the sphere of the topological mating by the semi-conjugacy. Applying Lemma 5.1 again, we get the equivalence between $N$ and $F_{y}$.

Concerning the study of $c_{2}^{-1}([N])$, we just need to see that for $j \in$ $\{2,3, \infty\}$, and $y, z \in Y_{j}, y \neq z$, the two graphs $T(y)$ and $T(z)$ cannot be homeomorphic by an orientation preserving map. In fact, both $y$ and $z$ have finite addresses relative to $u$ (i.e. a sequence of internal angles). Iterate the separating point between $T(y)$ and $T(z)$ until it becomes $u$ (or $v$ ), then iterate enough times until the two directions are separated by the union of the 0 -ray and the $1 / 2$-ray of $A_{u}$ (or $A_{v}$ ). This shows that $T(y)$ and $T(z)$ do not have the same cyclic order at $u$ (or $v$ ).

\section{Gluing captures and matings in the parameter space}

6.1. Regions and rays in parameter spaces. Tree classification. Recall that $g_{a}$ denotes the polynomial $z^{3}-3 a^{2} z+2 a^{3}+a$ and $K_{a}$ denotes the filled Julia set of $g_{a}$, more precisely

$$
K_{a}=\left\{z \in \mathbb{C} \mid g_{a}^{n}(z) \nrightarrow \infty \text { as } n \rightarrow \infty\right\} .
$$

In $[\mathrm{M} 1]$ and $[\mathrm{F}]$, the following notions and results are introduced and described in detail. Denote by $\mathcal{C}$ the connectedness locus of the parameter $a$-plane, i.e.

$$
\mathcal{C}=\left\{a \in \mathbb{C} \mid K_{a} \text { is connected }\right\} .
$$

The set $\mathcal{C}$ is shown on the left of Figure 1. Note that $-a$ is the unique free critical point of $g_{a}$, so the connectedness of $K_{a}$ is equivalent to $-a \in K_{a}$ (this is due to a classical result of Fatou: the Julia set of a polynomial is connected if and only if none of the critical points escapes to $\infty$ ). The set $\mathcal{C}$ is compact, full, and contains all postcritically finite polynomials $g_{a}$. 
There is a natural uniformizing mapping $\Phi$ from $\mathbb{C}-\mathcal{C}$ to $\mathbb{C}-\bar{D}$. It carries two foliations to $\mathbb{C}-\mathcal{C}$, one with external rays:

$$
\mathcal{R}_{\theta}=\left\{\Phi^{-1}\left(r e^{2 \pi i \theta}\right) \mid 1<r<\infty\right\},
$$

and one with equi-potentials: $\mathcal{E}_{r}=\left\{\Phi^{-1}\left(r e^{2 \pi i \theta}\right) \mid \theta \in \mathbb{T}\right\}$. The mapping $\Phi$ has the following property: if, for some $\theta$, the ray $\mathcal{R}_{\theta}$ passes through a point $a \in \mathbb{C}$, then in the dynamical plane of $g_{a}$, the external ray $R_{\theta}(a)$ for the polynomial $g_{a}$ is well defined above a certain potential level and passes through the co-critical point $2 a$ (recall that $g_{a}(-a)=g_{a}(2 a)$ ).

The $\operatorname{limit}_{\lim _{r \rightarrow 1}} \Phi^{-1}\left(r e^{2 \pi i \theta}\right)$ exists when $\theta$ is rational. We denote it by $\gamma_{\mathcal{C}}(\theta)$. It is considered as the landing point of $\mathcal{R}_{\theta}$. It is possible that $a$ is the landing point of more than one ray $\mathcal{R}_{\theta}$. We call the set $\left\{\theta \mid \gamma_{\mathcal{C}}(\theta)=a\right\}$ the set of external angles of $a$. If one external angle of $a$ is eventually fixed under angle tripling, then $a$ has no other external angle.

The union $\mathcal{R}_{1 / 3} \cup \mathcal{R}_{1 / 6} \cup \mathcal{R}_{2 / 3} \cup \mathcal{R}_{5 / 6}$ together with their landing points decompose the $a$-plane into three connected components. The one containing 0 coincides with the set

$$
\left\{a \in \mathbb{C} \mid \gamma_{a}(0), \gamma_{a}(1 / 2) \text { are well defined and distinct }\right\}
$$

(cf. [M1]). The two lines $\mathbb{R}$ and $i \mathbb{R}$ divide this component into four symmetrical parts. The lower right and the upper left quarters correspond to values of $a$ such that $\gamma_{a}(2 / 3) \in \partial A_{w}$.

Denote by $\mathcal{A}$ the closure of $\mathcal{C}$ intersecting the lower right quarter. Then the set $A \cup\{0\}$ is precisely the postcritically finite locus in $\mathcal{A}$ (see Figure 1).

Now let us return to the parameter space of Newton maps. Recall that in Section 1 we have defined a set $\Omega$ which is the fundamental domain of the space of cubic Newton maps.

Notation. Let $\Lambda$ be the set of $\lambda \in \Omega-\{ \pm 1 / 2\}$ such that $N_{\lambda}$ is postcritically finite (i.e. $x_{0}$ has finite orbit under $N_{\lambda}$ ). Note that for any $\lambda \in \Lambda$, the free critical point $x_{0}$ of $N_{\lambda}$ is not contained in $\left\{p_{1}, p_{2}, p_{3}\right\}$.

Here is a more precise statement of the main theorem.

ThEOREM 6.1. There is a well defined surjective mapping $\mathcal{M}: A \sqcup Y \rightarrow \Lambda$ with the following properties:

1. For $a \in A$ and $\lambda=\mathcal{M}(a)$, the mating $\mathbf{f} \Perp g_{a}$ is equivalent to $N_{\lambda}$, and $x_{0}$ is not a preimage of $p_{2}$ or $p_{3}$.

2. For $y \in Y$ and $\lambda=\mathcal{M}(y)$, the capture of $\mathbf{f}$ with co-critical point at $y$ is equivalent to $N_{\lambda}$, and $x_{0}$ is neither periodic nor a preimage of $p_{1}$.

3. $\mathcal{M}$ induces a bijection from $A \sqcup Y /\left\{\gamma_{\mathcal{C}}(\eta) \sim \gamma_{\mathbf{f}}(1-\eta)\right\}$ to $\Lambda$.

Proof. What we need is to combine Theorems 4.7 and 5.2. 
Just as in the decomposition of $Y$, we set

$A_{\mathrm{p}}=\{a \in A \mid-a$ is periodic $\}$,

$A_{\mathrm{sp}}=\{a \in A \mid-a$ is strictly preperiodic, but not eventually fixed $\}$,

$A_{1}=\{a \in A \mid-a$ is a preimage of $a\}$,

$A_{\infty}=\{a \in A \mid-a$ is eventually fixed, but not a preimage of $a\}$,

$\Lambda_{1}=\left\{\lambda \in \Lambda \mid x_{0}\right.$ is a preimage of $\left.p_{1}\right\}$,

$\Lambda_{\mathrm{p}}=\left\{\lambda \in \Lambda \mid x_{0}\right.$ is periodic for $\left.N_{\lambda}\right\}$,

$\Lambda_{\infty}=\left\{\lambda \in \Lambda \mid x_{0}\right.$ is a preimage of $\left.\infty\right\}$,

$\Lambda_{\mathrm{sp}}=\left\{\lambda \in \Lambda \mid x_{0}\right.$ is strictly preperiodic for $N_{\lambda}$, but not eventually fixed $\}$,

$\Lambda_{2}=\left\{\lambda \in \Lambda \mid x_{0}\right.$ is a preimage of $\left.p_{2}\right\}$,

$\Lambda_{3}=\left\{\lambda \in \Lambda \mid x_{0}\right.$ is a preimage of $\left.p_{3}\right\}$.

There is a bijection $h_{1}$ from (8) of Theorem 4.7 to $\Lambda_{\infty} \sqcup \Lambda_{\mathrm{p}} \sqcup \Lambda_{\mathrm{sp}} \sqcup \Lambda_{1}$ and a bijection $h_{2}$ from (10) of Theorem 5.2 to $\Lambda_{\infty} \sqcup \Lambda_{\mathrm{sp}} \sqcup \Lambda_{2} \sqcup \Lambda_{3}$ such that $h_{i}([N])=\lambda$ iff $N_{\lambda} \in[N](i=1,2)$.

For $a \in A$, set $\mathcal{M}(a)=h_{1}\left(m_{1}(a)\right)$. Then $\mathcal{M}(A)=\Lambda_{\infty} \sqcup \Lambda_{\mathrm{p}} \sqcup \Lambda_{\mathrm{sp}} \sqcup \Lambda_{1}$ according to Theorem 4.7 and the definition of $h_{1}$. Set $\lambda=\mathcal{M}(a)$. There is then a continuous semi-conjugacy $\phi$ from $\mathbf{f} \Perp g_{a}$ to $N_{\lambda}$ with ray-equivalence classes as fibers. Moreover, $\phi(w)=p_{1}$ and $\phi(-a)=x_{0}$. We also claim that $\phi$ gives rise to a conjugacy from the orbit of $-a$ to the orbit of $x_{0}$. This is trivial except in the case $a \in A_{\mathrm{sp}}$, when we just need to notice that each rayequivalence class contains at most one postcritical point (since each periodic non-fixed point in the Julia set of $\mathbf{f}$ has only one external ray landing at it). As a consequence, $\lambda \in \Lambda_{j}$ iff $a \in A_{j}, j \in\{\infty, \mathrm{p}, \mathrm{sp}, 1\}$.

Similarly, for $y \in Y$, set $\mathcal{M}(y)=h_{2}\left(c_{2}(y)\right)$, where $c_{2}$ is defined in Theorem 5.2. Then $\mathcal{M}(Y)=\Lambda_{\infty} \sqcup \Lambda_{\mathrm{sp}} \sqcup \Lambda_{2} \sqcup \Lambda_{3}$, and $\mathcal{M}(y) \in \Lambda_{j}$ iff $y \in Y_{j}$, $j \in\{\infty, \mathrm{sp}, 2,3\}$.

Denote by $\sim$ the equivalence relation in $A \sqcup Y$ generated by $\gamma_{\mathcal{C}}(\eta) \sim$ $\gamma_{\mathbf{f}}(1-\eta)$. We want to show that for each $\lambda \in \Lambda$, the set $\mathcal{M}^{-1}(\lambda)$ coincides with an equivalence class of $\sim$.

The class of a point in $A_{\mathrm{p}} \sqcup A_{1} \sqcup Y_{2} \sqcup Y_{3}$ consists of the point itself.

For $\lambda \in \Lambda_{\mathrm{p}} \cup \Lambda_{1}$, we have $\# \mathcal{M}^{-1}(\lambda)=\# \mathcal{M}^{-1}(\lambda) \cap A=1$. Similarly, for $\lambda \in \Lambda_{2} \cup \Lambda_{3}, \# \mathcal{M}^{-1}(\lambda)=\# \mathcal{M}^{-1}(\lambda) \cap Y=1$.

For $\lambda \in \Lambda_{\infty}, \mathcal{M}^{-1}(\lambda) \cap Y=\{y\}$ by Theorem 5.2. Moreover, $y \in Y_{\infty}$. It has then exactly two external angles $\eta, \eta^{\prime}$. Set $a=\gamma_{\mathcal{C}}(1-\eta)$ and $a^{\prime}=$ $\gamma_{\mathcal{C}}\left(1-\eta^{\prime}\right)$. Since $\eta, \eta^{\prime}$ are eventually fixed, the point $a$ (resp. $\left.a^{\prime}\right)$ has no other external angle. For $\mathbf{f} \Perp g_{a}$ (resp. $\mathbf{f} \Perp g_{a^{\prime}}$ ), the ray-equivalence class of the co-critical point contains the point $y$ in the Julia set of $\mathbf{f}$. Thus the graph $T(y)-\left\{y_{0}\right\}$ is injected into the sphere $S^{2} / \sim_{\text {ray }}$. By the uniqueness stated in Lemma 5.1 , both $\mathbf{f} \Perp g_{a}$ and $\mathbf{f} \Perp g_{a^{\prime}}$ are equivalent to $F_{y}$. So 
$a, a^{\prime} \in \mathcal{M}^{-1}(\lambda)$. But $\# \mathcal{M}^{-1}(\lambda) \cap A=2$ (Theorem 4.7), and finally we get $\mathcal{M}^{-1}(\lambda)=\left\{a, a^{\prime}, y\right\}$. This is an equivalence class of $\sim$.

For $\lambda \in \Lambda_{\mathrm{sp}}, \mathcal{M}^{-1}(\lambda) \cap A=\{a\}$ by Theorem 4.7. Moreover, $a \in A_{\mathrm{sp}}$. It has then finitely many external rays $\eta_{1}, \ldots, \eta_{k}$. Each of them is preperiodic but not eventually fixed under angle tripling. Set $y_{i}=\gamma_{\mathbf{f}}\left(1-\eta_{i}\right)$. This point has no other external angles. Moreover, $\mathbf{f} \Perp g_{a}$ and $F_{y_{i}}$ are equivalent. Thus $\mathcal{M}(a)=\mathcal{M}\left(y_{i}\right), i=1, \ldots, k$.

Conversely, let $y \in \mathcal{M}^{-1}(\lambda) \cap Y$. By Theorem 5.2 there is $a^{\prime} \in A$ such that $F_{y}, \mathbf{f} \Perp g_{a^{\prime}}$ and $N_{\lambda}$ are equivalent to each other. But $\mathcal{M}^{-1}(\lambda) \cap A$ contains only one element. So $a^{\prime}=a$. By the relationship between $a^{\prime}$ and $y$, we conclude that $y$ coincides with one of the $y_{i}$. Thus $\mathcal{M}^{-1}(\lambda)=$ $\left\{a, y_{1}, \ldots, y_{k}\right\}$. This is again an equivalence class of $\sim$.

Thus $\mathcal{M}^{-1}(\lambda)$ is an equivalence class for each $\lambda$. We are done.

Remark. According to the remarks following Theorems 4.7 and 5.2, one can extend $\mathcal{M}$ in a natural way to $(A \cup\{0\}) \sqcup(Y \cup\{u\})$ by setting $\mathcal{M}(0)=-1 / 2$ and $\mathcal{M}(u)=1 / 2$.

Proof of Corollary 1.4. Note that for $\lambda \in \operatorname{int}(\Omega)$, the three fixed critical points of $N_{\lambda}$ have a dynamical numbering $p_{1}, p_{2}$ and $p_{3}$ as in Section 2 , satisfying

$$
p_{1}=-1 / 2-\lambda, \quad p_{2}=-1 / 2+\lambda, \quad p_{3}=1 .
$$

To prove this, we show first that it is true for $\lambda \in \operatorname{int}(\Omega) \cap i \mathbb{R}$, with the help of symmetry, and then for all $\lambda \in \operatorname{int}(\Omega)$ since the rays $a_{1}(0), a_{2}(0)$, $a_{3}(0), a_{1}(1 / 2), a_{2}(1 / 2)$ and the points $\infty, \xi_{1}$ move holomorphically in $\lambda$ (see Section 2.1 for notations). Since $\mathcal{M}(A)$ contains $\lambda$ such that $x_{0}$ is attracted by $p_{1}$ or $x_{0}$ is periodic, $\mathcal{M}(A) \supset \Lambda \cap\left(C^{-} \cup D\right)$ by definition of $C^{-}$and $D$. The rest is similar.

Conjecture. The mapping $\mathcal{M}$ extends to a homeomorphism

$$
\mathcal{A} \sqcup \mathcal{Y} /\left\{\gamma_{\mathcal{C}}(\eta) \sim \gamma_{\mathbf{f}}(1-\eta)\right\} \rightarrow \Omega
$$

satisfying $\mathcal{M}\left(0_{\mathcal{A}}\right)=-1 / 2, \mathcal{M}\left(u_{\mathcal{Y}}\right)=1 / 2$ and $\mathcal{M}\left(\left[\gamma_{\mathbf{f}}(1 / 6)\right]\right)=0$.

We can now classify all postcritically finite Newton maps by topologically finite graphs embedded in $S^{2}$. The importance of these graphs is that they use finite information to describe the structure of the Fatou components of $\overline{\mathbb{C}}-J_{N}$, of which there are in general countablely many. We will use the property that each $g_{a}, a \in A$, has a forward invariant topologically finite tree, the Hubbard tree.

Denote by $H_{a}^{1}$ the convex hull in $K_{a}$ of the Hubbard tree and $\gamma_{a}(0)$ (the landing point of the external 0-ray), and by $T(a)$ the tree $H_{\mathbf{f}} \sqcup H_{a}^{1} /\left\{\gamma_{\mathbf{f}}(0) \sim\right.$ $\left.\gamma_{a}(0)\right\}$ embedded in $S^{2}$ so that the cyclic order around $\gamma_{\mathbf{f}}(0)$ is $u, v, H_{a}^{1}$. 
Corollary 6.2. Denote by $\mathcal{T}$ the set of embedded graphs

$$
\{T(a) \mid a \in A\} \sqcup\{T(y) \mid y \in Y\} .
$$

Then $\mathcal{M}$ induces a surjective mapping $\mathcal{M}_{\mathcal{T}}$ from $\mathcal{T}$ to $\Lambda$. Moreover, for $T \in \mathcal{T}$ and $\lambda=\mathcal{M}_{\mathcal{T}}(T)$, the Newton map $N_{\lambda}$ has a forward invariant graph $H$ homeomorphic to $T$, containing the postcritical set of $N$. And $\left.N\right|_{H}$ is conjugate to the induced self-mapping on $T$ (which is $\mathbf{f} \Perp g_{a}$ in case $T=T(a)$ and $\mathbf{f}$ in case $T=T(y))$. Furthermore, the fibers of $\mathcal{M}_{\mathcal{T}}$ are completely determined by the fibers of $\mathcal{M}$.

COROLlary (of the corollary). Each postcritically finite Newton map has a forward invariant topologically finite graph, containing the postcritical set.

6.2. Finding Head's angles from captures and matings. In Section 2.3 we have seen the importance of Head's angles and how (conjecturally) they classify the components of $\Omega-\overline{B_{0} \cup G_{0}}$. Here we try to determine Head's angles from matings and captures.

Denote by $\mathcal{H}_{0}$ the connected component of the interior of $\mathcal{C}$ containing 0 . It is a hyperbolic component. We call it the main hyperbolic component of the $a$-plane.

There is a natural uniformizing mapping $\Psi$ from the set $\left\{a^{2} \mid a \in \mathcal{H}_{0}\right\}$ to $D$. We denote again by $\Psi$ the composed mapping $a \mapsto \Psi\left(a^{2}\right)$ from $\mathcal{H}_{0}$ to $D$. This mapping carries two foliations to $\mathcal{H}_{0}-\{0\}$, one by internal rays, the other by equi-potentials. Note that for any $t \in \mathbb{T}$ there are two rays with angle $t$. By the construction of $\Psi$, this mapping has the following property: if a point $a \in \mathcal{H}_{0}$ is on the internal ray of angle $t$, then in the dynamical plane of $g_{a}$, the internal ray $R_{t}(a)$ in the immediate basin of the fixed critical point $a$ is well defined below a certain potential level, and passes through $2 a$, the co-critical point of $g_{a}$.

The mapping $\Psi$ extends to a continuous mapping from $\mathbb{C}$ to $\bar{D}$ which is constant on each connected component of $\mathcal{C}-\overline{\mathcal{H}}_{0}$ (cf. [M1] and [F]).

Denote by $r_{t}$ the set $\left\{\Psi^{-1}\left(r e^{2 \pi i t}\right) \mid r \in\left[0,1[\} \cap \mathcal{A}\right.\right.$. Denote by $\mathcal{A}_{t}$ the set $\left\{\Psi^{-1}\left(e^{2 \pi i t}\right)\right\} \cap \mathcal{A}$. It is called the t-limb of $\mathcal{A}$.

The set $\mathcal{A}$ then coincides with the set $r_{1 / 2} \cup r_{1} \cup \bigcup_{t \in] 1 / 2,1[}\left(r_{t} \cup \mathcal{A}_{t}\right)$. It is also the intersection of the connectedness locus $\mathcal{C}$ with the sector region bounded by the curve $\mathcal{R}_{5 / 6} \cup r_{1 / 2} \cup r_{1} \cup \mathcal{R}_{0}$. Similarly, the set $\mathcal{Y}-\left\{\gamma_{\mathbf{f}}(1 / 6)\right\}$ coincides with the set of points whose internal angle relative to $u$ is in $] 0,1 / 2[$.

The subset $\mathcal{S}$ of $\mathbb{T}$ was defined and studied in Subsection 2.2. The Head's angle for a Newton map was defined and studied in Subsection 2.3.

Proposition 6.3. Given $I=] \beta, \alpha[$ a connected component of $\mathbb{T}-\mathcal{S}$ with $\alpha, \beta$ rational and $0<\beta<\alpha<1 / 2$, denote by $\Lambda_{[\beta, \alpha]}$ the set of $\lambda \in \Lambda$ such 
that the Head's angle of $N_{\lambda}$ is contained in $\{\beta, \alpha\}$. We have

$$
\mathcal{M}^{-1}\left(\Lambda_{[\beta, \alpha]}\right)=\bigcup_{t \in[\beta, \alpha]}\left(A \cap \mathcal{A}_{t+1 / 2}\right) \sqcup \bigcup_{t \in[\beta, \alpha]}\left(Y \cap \mathcal{Y}_{1 / 2-t}\right) .
$$

P r o of. We can easily check the following two facts using Milnor's algorithm ([M1], Lemma 2.5).

1. For any $t \in\left[\beta, \alpha\left[\cap \mathbb{Q}\right.\right.$, and any $a \in A \cap \mathcal{A}_{t+1 / 2}$, the point $x_{\alpha} \in \partial A_{w}$ of internal angle $\alpha$, for the polynomial $g_{a}$, has a unique external angle $\xi(\alpha)$ depending neither on $a$ nor on $t$. For $t=\alpha$ and $a \in A \cap \mathcal{A}_{t+1 / 2}, x_{\alpha}$ has two external angles with the larger one equal to $\xi(\alpha)$.

2. For any $t \in] \beta, \alpha] \cap \mathbb{Q}$, and any $a \in A \cap \mathcal{A}_{t+1 / 2}$, the point $x_{\beta} \in \partial A_{w}$ of internal angle $\beta$, for the polynomial $g_{a}$, has a unique external angle $\xi(\beta)$ depending neither on $a$ nor on $t$. For $t=\beta$ and $a \in A \cap \mathcal{A}_{t+1 / 2}, x_{\beta}$ has two external angles with the smaller one equal to $\xi(\beta)$.

It is not difficult to check that the external ray $R_{\mathbf{f}}(1-\xi(\alpha))$ of $\mathbf{f}$ lands at a point of $\partial A_{u}$. Hence for any $a$ in $\bigcup_{t \in] \beta, \alpha]}\left(A \cap \mathcal{A}_{t+1 / 2}\right)$, and $\phi$ the semi-conjugacy in Theorem 4.7, we have

$$
\phi\left(x_{\alpha}\right) \in \phi\left(\bar{A}_{w}\right) \cap \phi\left(\bar{A}_{u}\right) .
$$

Since $\phi$ is conformal in $\operatorname{int}\left(K_{\mathbf{f}}\right) \cup \operatorname{int}\left(K_{a}\right)$, it maps internal rays of $\mathbf{f}$ and $g_{a}$ onto internal rays of $N_{\mathcal{M}(a)}$ with the same angle. To see that the Head's angle of $N_{\mathcal{M}(a)}$ is $\alpha$ we only need to show that $\alpha$ is the minimal angle such that (11) holds. Suppose that the Head's angle is smaller than $\alpha$. Then it should be smaller than or equal to $\beta$ as it should be in $\mathcal{S}$. Figure 9 shows that for any $s \leq \beta,\left[x_{s}\right]_{F} \cap \bar{A}_{u}=\emptyset$, where $x_{s} \in \partial A_{w}$ is the point with internal angle $s$.

There is a unique point $a$ in $A \cap \mathcal{A}_{\beta+1 / 2}$. The Head's angle of $N_{\mathcal{M}(a)}$ is $\beta$. The details are omitted.

In the graph $T(y)$, the internal angle of $y_{0}$ relative to $u$ is $s$ when $s-1 / 2$ is the internal angle of $y$. By assumption, $s \in[1-\alpha, 1-\beta]$. The unique Jordan curve contained in $T(y) /\left\{y_{0} \sim y_{0}^{\prime}\right\}$ can be seen as a Jordan curve $\gamma$ in the dynamics of $N=N_{\mathcal{M}(y)}$. Recall that $a_{i}(t)$ denotes the $t$-ray of the immediate attracting basin $A_{i}$ of $p_{i}$ for $N$. We have $\gamma \cap A_{1}=\emptyset$, and $a_{2}(s) \subset \gamma$. Let $t$ be the Head's angle of $N$, and set $s^{\prime}=1-t$. It is enough to show that $s^{\prime}=1-\alpha$ if $s \neq 1-\beta$ and $s^{\prime}=1-\beta$ if $s=1-\beta$. The details are omitted.

This shows that

$$
\mathcal{M}\left(\bigcup_{t \in[\beta, \alpha]}\left(A \cap \mathcal{A}_{t+1 / 2}\right) \sqcup \bigcup_{t \in[\beta, \alpha]}\left(Y \cap \mathcal{Y}_{1 / 2-t}\right)\right) \subset \Lambda_{[\beta, \alpha]} .
$$

The surjectivity is a consequence of the surjectivity of $\mathcal{M}$. We omit the details again. 


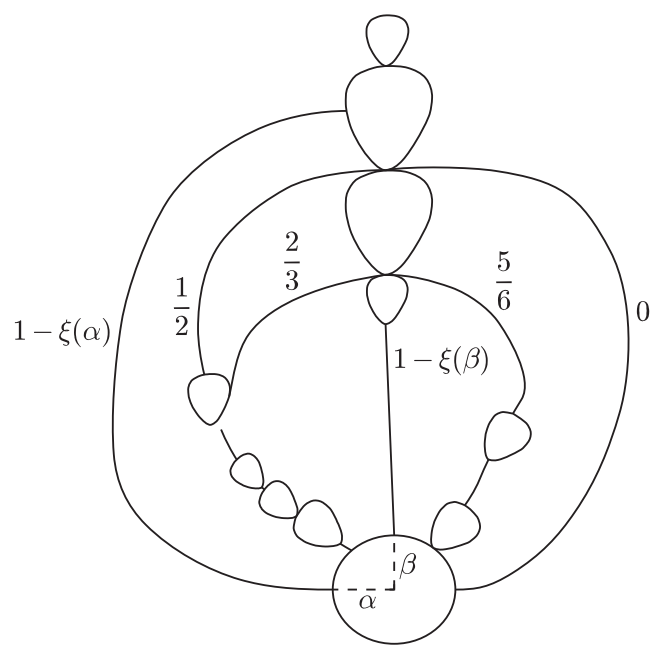

Fig. 9. $\alpha$ is minimal

R e mark. Starting from external angles of $a \in A$ or of $y \in Y$, we can find their internal angles. This is then enough to find the Head's angle of $N_{\mathcal{M}(a)}$ or $N_{\mathcal{M}(y)}$ by the above proposition. Given $t \in \mathcal{S}$ with even denominator (for example $t=1 / 6$ ), there is $a \in A$ with internal angle $t$. One can show similarly that $N_{\mathcal{M}(a)}$ has Head's angle $t$.

\section{References}

[B] P. Blanchard, Complex analytic dynamics on the Riemann sphere, Bull. Amer. Math. Soc. 11 (1984), 85-141.

[CGS] J. Curry, L. Garnett and D. Sullivan, On the iteration of rational functions: Computer experiments with Newton's method, Comm. Math. Phys. 91 (1983), 267-277.

[D1] A. Douady, Systèmes dynamiques holomorphes, Séminaire Bourbaki, 35e année, 1982-1983, exp. no. 599, 1982.

[D2] - Algorithm for computing angles in the Mandelbrot set, in: Chaotic Dynamics and Fractals, M. F. Barnsley and S. G. Demko (eds.), Academic Press, New York, $1986,155-168$.

[D3] - Chirurgie sur les applications holomorphes, in: Proc. Internat. Congress Math., Berkeley, Calif., 1986, 724-738 (English version: preprint MSRI, 1986).

[DH1] A. Douady et J. H. Hubbard, Étude dynamique des polynômes complexes, I et II, avec la collaboration de P. Lavaurs, Tan Lei et P. Sentenac, Publication d'Orsay 84-02, 85-04, 1984/1985.

[DH2] - - - A proof of Thurston's topological characterization of rational functions, Acta Math. 171 (1993), 263-297.

[DH3] - - - On the dynamics of polynomial-like mappings, Ann. Sci. École Norm. Sup. (4) 18 (1985), 287-343. 
[F] D. Faught, Local connectivity in a family of cubic polynomials, Ph.D. thesis, Cornell Univ., Ithaca, N.Y., 1992.

[Ha] F. von Haeseler, Über Attraktionsgebiete superattraktiver Zykle, Ph.D. thesis, Bremen Univ., Bremen, 1985.

[HP] F. von Haeseler and H.-O. Peitgen, Newton's method and complex dynamical systems, Acta Appl. Math. 13 (1988), 3-58.

[He] J. Head, The combinatorics of Newton's method for cubic polynomials, Ph.D. thesis, Cornell Univ., Ithaca, N.Y., 1987.

[Le] S. Levy, Critically finite rational maps, Ph.D. Thesis, Princeton Univ., Princeton, N.J., 1985.

[Me] H.-G. Meier, On the connectedness of the Julia-set for rational functions, preprint, Aachen Univ., 1989.

[M1] J. Milnor, On cubic polynomials with periodic critical point (very rough draft), 5-28-91.

[M2] -, Dynamics in one complex variable: Introductory lectures, preprint Stony Brook 1990-5.

[Prz] F. Przytycki, Remarks on the simple connectedness of basins of sinks for iterations of rational maps, in: Dynamical Systems and Ergodic Theory, K. Krzyżewski (ed.), PWN-Polish Sci. Publ., 1989, 229-235.

[R1] M. Rees, A partial description of parameter space of rational maps of degree two: Part I, Acta Math. 168 (1992), 11-87.

[R2] -, A partial description of parameter space of rational maps of degree two: Part II, Proc. London Math. Soc. (3) 70 (1995), 644-690.

[R3] - Realization of matings of polynomials as rational maps of degree two, manuscript, 1986.

[Sa] D. Saupe, Discrete versus continuous Newton's method: A case study, Acta Appl. Math. 13 (1988), 59-80.

[Sh1] M. Shishikura, The connectivity of the Julia set of rational maps and Fixed points, preprint, I.H.E.S., Bures-sur-Yvette, 1990.

[Sh2] - On a theorem of M. Rees for matings of polynomials, preprint, I.H.E.S., Bures-sur-Yvette, 1990.

[ST] M. Shishikura and L. Tan, A family of cubic rational maps and matings of cubic polynomials, preprint 88-50, Max-Planck-Institut für Mathematik, Bonn.

[Ta] L. Tan, Matings of quadratic polynomials, Ergodic Theory Dynam. Systems 12 (1992), 589-620.

[TY] L. Tan and Y. C. Yin, Local connectivity of the Julia set for geometrically finite rational maps, Sci. in China (Ser. A) 39 (1) (1996), 39-47.

[Th] W. Thurston, The combinatorics of iterated rational maps, preprint, Princeton Univ., Princeton, N.J., 1983.

[W] B. Wittner, On the bifurcation loci of rational maps of degree two, Ph.D. thesis, Cornell Univ., Ithaca, N.Y., 1986.

Department of Mathematics

University of Warwick

Coventry CV4 7AL

United Kingdom

E-mail: tanlei@maths.warwick.ac.uk 